\title{
Design of meta-heuristic computing paradigms for Hammerstein identification systems in electrically stimulated muscle models
}

\author{
Ammara Mehmood ${ }^{1}$, Aneela Zameer ${ }^{2}$, Naveed Ishtiaq Chaudhary ${ }^{3}$, Sai Ho Ling, \#, Muhammad \\ Asif Zahoor Raja ${ }^{5}$ \\ ${ }^{1}$ Department of Electrical Engineering, Pakistan Institute of Engineering and Applied Sciences, Nilore, \\ Islamabad, Pakistan \\ ms_mehmud16@pieas.edu.pk; \\ ${ }^{2}$ Department of Computer and Information Sciences, Pakistan Institute of Engineering and Applied \\ Sciences, Nilore, Islamabad, Pakistan \\ aneelaz@pieas.edu.pk \\ ${ }^{3}$ Department of Electrical Engineering, International Islamic University, Islamabad, Pakistan \\ naveed.ishtiaq@iiu.edu.pk
${ }^{4}$ School of Biomedical Engineering, Centre for Health Technologies, Department of Engineering and IT, University of Technology, Sydney, NSW, Australia
Steve.Ling@uts.edu.au
${ }^{\#}$ Corresponding author
${ }^{3}$ Department of Electrical and Computer Engineering, COMSATS University Islamabad, Attock Campus, Attock, Pakistan
muhammad.asif@ciit-attock.edu.pk

\begin{abstract}
In this study, a novel application of differential evolution (DE) based computational heuristics is proposed for identification of Hammerstein structures representing the electrically stimulated muscle (ESM) models as a part of rehabilitation interventions for the stock patient to prevent the post spinal cord injury atrophy. The strength of approximation theory is incorporated for defining the fitness function for ESM system based on mean square deviation between actual and estimated responses. DE, genetic algorithms (GAs), particle swarm optimization (PSO), pattern search (PS) and simulated annealing (SA) are used as optimization mechanisms to identify the ESM models with input nonlinearities of sigmoidal, polynomial and spline kernels for noiseless and noisy environments. Comparative studies based on detailed statistics establish the worth of DE based heuristics over its counterparts GAs, PSO, PS and SA in terms of accuracy, convergence, robustness and efficiency for identification of ESM models arising in rehabilitation of the stock patients.
\end{abstract}

Keywords: Parameter Estimation; Electrically Stimulated Muscle Models; Differential Evolution; Genetic Algorithms; Nonlinear Hammerstein Systems; Evolutionary Computing.

\section{Introduction}

The spinal cord injury (SCI) has significant impact on volitional activities of muscle and may effect in reducing the cross-sectional area of the muscle nearly by $45 \%$ during the first few weeks of SCI [1]. The individuals suffering from SCI are more at the risk of lifetime than non-SCI patients 
[2]. Rehabilitation interventions are essential for prevention of post SCI atrophy in order to enhance the effectiveness of muscle strength. Electrical muscle stimulation is a viable post SCI strategy with objective to trigger muscle hypertrophy for enhancing density of muscle with respect to its healthy level and accordingly improves torque output and combats fatigue [3-4]. Electrical muscle stimulation is also helpful in restoration of routine functions such as stretching, bending and reaching [5].

Design of controllers is required to facilitate the application of electrical stimulation for rehabilitation of stroke patients with accurate modeling of electrically stimulated muscle (ESM). Modeling of ESM has been a challenging task and research community has paid special attention to model muscle dynamics, considering both isometric and non-isometric conditions. These models are categorized into Hill-type [6], mathematical [7-8], and block oriented Hammerste inWiener structures [9-10]. After detailed experimentations carried out at the rehabilitation center, Southampton University, it was determined that nonlinear Hammerstein system best describes the muscle activation system having a well-defined structure and different estimation techniques for each block [11]. Hammerstein structure comprises of two blocks; first part represents nonlinear systems while subsequent block for linear characteristics [12-13]. In relation to the ESM model, static nonlinearity characterizes the isometric recruitment curve which is a gain relation between the activation levels of the stimulus and output torque, assuming the fixed length of the muscle [12]. While the linear block represents ESM contraction dynamics that combines with isometric recruitment curve to produce average torque [12]. After accurate modeling, the next phase is the parameter estimation of Hammerstein system representing the ESM model.

Researchers have great contributions to develop reliable mechanisms for identification of Hammerstein models [14-16] including, modified gradient descent and recursive least squares strategies incorporating hierarchical approach [17-18]. Furthermore, multi innovation method [1920], auxiliary mechanism [21] and parameter separation theory [22] have also been proposed. In the recent years, fractional order gradient schemes were effectively employed for parameter identification of nonlinear Hammerstein models [23-28]. These all are deterministic procedures with their own advantages, applications, and limitations while the stochastic techniques [29-34] are not extensively exploited for the parameter estimation of input nonlinear Hammerstein systems as yet. Few potential applications of these methodologies based on exploration and exploitation of artificial neural networks (ANNs), differential evolution (DE) and genetic algorithms (GAs) include fractional order systems [35-36], nonlinear singularly perturb systems [37], nonlinear pantograph systems [38], nonlinear prey-predator models [39], nonlinear chaotic systems [40-41], models of nonlinear optics [42], random matrix theory based application [43], thin film flow systems [44], thermal analysis of porous fin model [45], input nonlinear control autoregressive systems [46-47], active noise control systems [48-49] and control autoregressive moving average systems [50]. Beside these recently stochastic solvers are used to address viably the optimization problems arising in various domains such as astrophysics [51-52], atomic physics [53-54], plasma physics [55-56], thermodynamics [57], mechanics [58-59], nanotechnology [60-61], electric circuits [62-63], energy [64-65], power [66-67], finance [68-69], economics [70-71] and bioinformatics [72-73]. These are the motivational aspects for authors to investigate in the domain of parameter estimation of input nonlinear Hammerstein system representing the electrically stimulated muscle model scenarios through DE, GAs and particle swarm optimization (PSO) as global search mechanisms, as well as pattern search (PS) and simulated annealing (SA).

The salient features of the research study are briefly presented as follows: 
- Novel exploitation of computational heuristics through differential evolution in the field of biomedical signal processing to estimate the parameters of electrically stimulated muscle model for rehabilitation of stroke patients.

- Hammerstein structure based ESM model with cubic-spline, sigmoid, and polynomial nonlinearities in the dynamics are effectively identified with respect to true parameters of the system.

- The performance of DE based scheme in terms of accuracy, stability and robustness is invariably better from GAs, PSO, PS and SA for noiseless as well as low and high noisy scenarios of all examples of ESM models.

- The consistency of the worthy identification of differential evolution is indorsed through statistical observations on multiple trails by means of precision and complexity indices.

Organization of paper is as follows: the designed methodology in the form of mathematical formulation for the ESM model and its optimization methodology based on DE and GAs is presented in Section II, in Section III performance indices are narrated in details, in Section IV statistical analyses on large dataset is presented, while in the last Section a brief list of conclusions is provided.

\section{Design methodology}

The design procedure is presented here that consist of two phases; in the first phase, an overview of electrically stimulated muscle (ESM) models along with formulation of objective function is given, while in the second phase, a brief overview of the optimization solver based on DE and GAs is provided for parameter identification problem of ESM. The schematic of workflow proposed in the present study is illustrated in Fig. 1.

\subsection{Electrically stimulated muscle modelling}

The modeling of ESM dynamics is carried out through a nonlinear Hammerstein structure in discrete-time is shown in Fig. 2, and is mathematically written as [11-13].

$$
x(t)=\frac{P(z)}{Q(z)} g(u(t))+\frac{1}{Q(z)} n(t)
$$

Here, $u(t)$ represents stimulation input, $x(t)$ denotes system output and $n(t)$ stands for disturbance noise. $P(z)$ is the polynomial defining poles, while $Q(z)$ is the polynomials representing zeros:

$$
\begin{aligned}
& P(z)=p_{1} z^{-1}+p_{2} z^{-2}+\cdots+p_{n_{p}} z^{-n_{p}}, \\
& Q(z)=1+q_{1} z^{-1}+q_{2} z^{-2}+\cdots+q_{n_{q}} z^{-n_{q}} .
\end{aligned}
$$

Activation function representing nonlinear system input of muscle dynamics is $g(u) \cdot g(u)$ for polynomial nature of input is expressed as:

$$
g(u)=\gamma_{1} u+\gamma_{2} u^{2}+\gamma_{3} u^{3}+\cdots+\gamma_{m} u^{m}
$$


Here, $\gamma_{1}, \gamma_{2}, \gamma_{3}, \cdots, \gamma_{m}$ are coefficients of polynomial and $\mathrm{m}$ is the degree of the polynomial.

In higher order derivative system might be at risk of oscillations. Another option is spline function but their derivatives are not continuous at their break points or knots. So, cubic spline is used to model the nonlinear block. Additionally, sigmoid functions are good alternatives. Here, $f(u)$ for sigmoid and cubic-spline nature of input are expressed as: [11]:

$$
\begin{aligned}
& g(u)=\gamma_{1} \cdot \frac{e^{\gamma_{2} u}-1}{e^{\gamma_{2} u}+\gamma_{3}}, \\
& g(u)=\sum_{i=1}^{m-2} \gamma_{i}\left|u-u_{i+1}\right|^{3}+\gamma_{m-1}+\gamma_{m} u+\gamma_{m+1} u^{2}+\gamma_{m+2} u^{3}
\end{aligned}
$$

Taking one knot at 150 in the cubic-spline activation function, equation (6) takes the form:

$$
g(u)=\gamma_{1}|u-150|^{3}+\gamma_{2}+\gamma_{3} u+\gamma_{4} u^{2}+\gamma_{5} u^{3}
$$

In order to define the cost function, let define the true parameter vector of ESM model is

$$
\vartheta=\left[\vartheta_{l}, \vartheta_{n}\right]
$$

here $\vartheta_{l}$ represents parameter for linear and $\vartheta_{n}$ for nonlinear blocks of ESM model, respectively. $\vartheta_{l}$ is described as:

$$
\vartheta_{l}=\left[q_{1}, q_{2}, \ldots, q_{n_{q}}, p_{1}, p_{2}, \ldots, p_{n_{p}}\right]
$$

The parameters related to nonlinear block are denoted by $\vartheta_{n}$ and by assuming polynomial (4), sigmoid (5) and cubic-splines (6), $\vartheta_{n}$ can be written as [11]:

$$
\vartheta_{n}= \begin{cases}{\left[\gamma_{1}, \gamma_{2}, \ldots, \gamma_{m}\right],} & \text { polynomial case } \\ {\left[\gamma_{1}, \gamma_{2}, \gamma_{3}\right],} & \text { sigmoid case } \\ {\left[\gamma_{1}, \gamma_{2}, \ldots, \gamma_{5}\right]} & \text { spline case }\end{cases}
$$

The output of ESM models of cubic-spline, polynomial, and sigmoid functions are expressed, as:

$$
\begin{aligned}
& x(t)=-\left(\sum_{i=1}^{n_{q}} q_{i} z^{-i}\right) x(t)+\left(\sum_{i=1}^{n_{p}} p_{i} z^{-i}\right) \sum_{i=1}^{m-2}\left(\begin{array}{l}
\gamma_{i}\left|u(t)-u_{i+1}(t)\right|^{3}+\gamma_{m-1}+ \\
\gamma_{m} u(t)+\gamma_{m+1} u^{2}(t)+\gamma_{m+2} u^{3}(t)
\end{array}\right)+n(t) . \\
& x(t)=-\left(\sum_{i=1}^{n_{q}} q_{i} z^{-i}\right) x(t)+\left(\sum_{i=1}^{n_{p}} p_{i} z^{-i}\right)\left(\gamma_{1} \cdot \frac{e^{\gamma_{2} u(t)}-1}{e^{\gamma_{2} u(t)}+\gamma_{3}}\right)+n(t),
\end{aligned}
$$




$$
x(t)=-\left(\sum_{i=1}^{n_{q}} q_{i} z^{-i}\right) x(t)+\left(\sum_{i=1}^{n_{p}} p_{i} z^{-i}\right)\left(\gamma_{1} u(t)+\gamma_{2} u^{2}(t)+\cdots+\gamma_{m} u^{m}(t)\right)+n(t),
$$

Corresponding output from ESM for cubic-spline, sigmoid and polynomial at $k$ instances are written as:

$$
\begin{aligned}
& x_{k}=-\left(\sum_{i=1}^{n_{q}} q_{i} z^{-i}\right) x_{k}+\left(\sum_{i=1}^{n_{p}} p_{i} z^{-i}\right) \sum_{i=1}^{m-2}\left(\begin{array}{l}
\gamma_{i}\left|u_{k}-u_{k, i+1}\right|^{3}+\gamma_{m-1}+ \\
\gamma_{m} u_{k}+\gamma_{m+1} u_{k}^{2}\left(t_{k}\right)+\gamma_{m+2} u_{k}^{3}
\end{array}\right)+n_{k} . \\
& x_{k}=-\left(\sum_{i=1}^{n_{q}} q_{i} z^{-i}\right) x_{k}+\left(\sum_{i=1}^{n_{p}} p_{i} z^{-i}\right)\left(\begin{array}{l}
\gamma_{1} u_{k}+\gamma_{2} u_{k}^{2}+ \\
\cdots+\gamma_{m} u_{k}^{m}
\end{array}\right)+n_{k}, \\
& x_{k}=-\left(\sum_{i=1}^{n_{q}} q_{i} z^{-i}\right) x_{k}+\left(\sum_{i=1}^{n_{p}} p_{i} z^{-i}\right)\left(\begin{array}{l}
\gamma_{1} u_{k}+\gamma_{2} u_{k}^{2}+ \\
\cdots+\gamma_{m} u_{k}^{m}
\end{array}\right)+n_{k},
\end{aligned}
$$

for $x_{k}=x\left(t_{k}\right), u_{k}=u\left(t_{k}\right), n_{k}=n\left(t_{k}\right)$ in case of $k=1,2, \ldots, K$, where $\mathrm{K}$ is total number of instances.

Now task is to obtain the cost functions of ESM models by utilizing the formulation of mean square errors, $\varepsilon$ as:

$$
\varepsilon=\varepsilon_{1}+\varepsilon_{2}
$$

here, error function $\varepsilon_{1}$ is variation between the estimated $\boldsymbol{x}=\left[x_{1}, x_{2}, \ldots, x_{K}\right]$ and the desired response $\hat{\boldsymbol{x}}=\left[\hat{x}_{1}, \hat{x}_{2}, \ldots, \hat{x}_{K}\right]$, while and $\varepsilon_{2}$ is the mean square difference between estimated $\hat{\vartheta}$ and desired $\vartheta$ weight vectors. The mathematical expression for $\varepsilon_{1}$ is given as:

$$
\varepsilon_{1}=\frac{1}{K} \sum_{k=1}^{K}\left(x_{k}-\hat{x}_{k}\right)^{2}
$$

Here, the $k^{\text {th }}$ output of ESM model is $x_{k}$ as in (14-16), while the $\hat{x}_{k}$ is the $k^{\text {th }}$ output of estimated output response in terms of $\hat{\vartheta}$ with polynomial $g(u)$ :

$$
\begin{aligned}
& \hat{\vartheta}=\left[\hat{\vartheta}_{l}, \hat{\vartheta}_{n}\right]=\left[\hat{q}_{1}, \hat{q}_{2}, \ldots, \hat{q}_{n_{q}}, \hat{p}_{1}, \hat{p}_{2}, \ldots, \hat{p}_{n_{p}}, \hat{\gamma}_{1}, \hat{\gamma}_{2}, \ldots, \hat{\gamma}_{m}\right], \\
& \hat{x}_{k}=-\left(\sum_{i=1}^{n_{q}} q_{i} z^{-i}\right) \hat{x}_{k}+\left(\sum_{i=1}^{n_{p}} p_{i} z^{-i}\right)\left(\begin{array}{l}
\gamma_{1} u_{k}+\gamma_{2} u_{k}^{2}+ \\
\cdots+\gamma_{m} u_{k}^{m}
\end{array}\right), \quad k=1,2, \ldots, K
\end{aligned}
$$

Accordingly, the estimated response $\hat{\boldsymbol{x}}$ with approximate parameter vector $\hat{\boldsymbol{\vartheta}}$ by taking sigmo id as basis function is written as: 


$$
\begin{aligned}
& \hat{\boldsymbol{\vartheta}}=\left[\hat{\boldsymbol{\vartheta}}_{l}, \hat{\vartheta}_{n}\right]=\left[\hat{q}_{1}, \hat{q}_{2}, \ldots, \hat{q}_{n_{q}}, \hat{p}_{1}, \hat{p}_{2}, \ldots, \hat{p}_{n_{p}}, \hat{\gamma}_{1}, \hat{\gamma}_{2}, \ldots, \hat{\gamma}_{m}\right]
\end{aligned}
$$

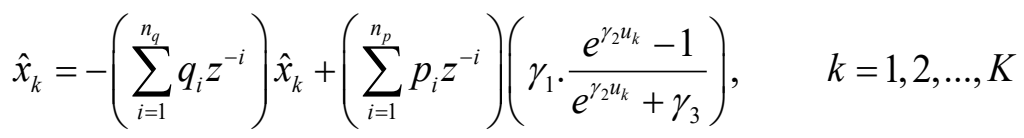

Similarly, the estimated response $\hat{\boldsymbol{x}}$ with $\hat{\boldsymbol{\vartheta}}$ for cubic-spline $g(u)$ is described as:

$$
\begin{aligned}
& \hat{\boldsymbol{\vartheta}}=\left[\hat{\boldsymbol{\vartheta}}_{l}, \hat{\boldsymbol{\vartheta}}_{n}\right]=\left[\hat{q}_{1}, \hat{q}_{2}, \ldots, \hat{q}_{n_{q}}, \hat{p}_{1}, \hat{p}_{2}, \ldots, \hat{p}_{n_{p}}, \hat{\gamma}_{1}, \hat{\gamma}_{2}, \ldots, \hat{\gamma}_{m}\right], \\
& \hat{\boldsymbol{x}}_{k}=-\left(\sum_{i=1}^{n_{q}} q_{i} z^{-i}\right) \hat{\boldsymbol{x}}_{k}+\left(\sum_{i=1}^{n_{p}} p_{i} z^{-i}\right) \sum_{i=1}^{m-2}\left(\begin{array}{l}
\gamma_{i}\left|u_{k}-u_{k, i+1}\right|^{3}+\gamma_{m-1}+ \\
\gamma_{m} u_{k}+\gamma_{m+1} u_{k}^{2}+\gamma_{m+2} u_{k}^{3}
\end{array}\right), \quad k=1,2, \ldots, K
\end{aligned}
$$

The error function $\varepsilon_{2}$ is mathematically described as a mean squared error between the estimated and desired ESM parameters:

$$
\varepsilon_{2}=\frac{1}{N} \sum_{k=1}^{N}\left(\vartheta_{k}-\hat{\vartheta}_{k}\right)^{2}
$$

Here, $N$ represents the total number of elements in the parameter vector, $\vartheta_{k}$ and $\hat{\vartheta}_{k}$ denotes the $k^{\text {th }}$ entity of desired and estimated weight vector, respectively. For input nonlinear functions $g(u)$ , the parameter $N$ is $n_{q}+n_{p}+m$ for polynomial, $n_{q}+n_{p}+3$ for sigmoid, and $n_{q}+n_{p}+m$ for cubicspline. So the objective function (17) using (18) and (25) is written as:

$$
\varepsilon=\frac{1}{K} \sum_{k=1}^{K}\left(x_{k}-\hat{x}_{k}\right)^{2}+\frac{1}{N} \sum_{k=1}^{N}\left(\vartheta_{k}-\hat{\vartheta}_{k}\right)^{2}
$$

Next step is to utilize the heuristic schemes to obtain the solution of the ESM models (26) by finding the optimal parameters of $\hat{\vartheta}$ so that $\varepsilon \rightarrow 0$, as error approaches zero, estimated solution approaches the desired solution, i.e., $\hat{\boldsymbol{x}} \rightarrow \boldsymbol{x}$, therefore consequently, the estimated parameter vector approaches its optimal, i.e., $\hat{\vartheta} \rightarrow \vartheta$.

\subsection{Optimization Mechanism for ESMs}

A brief introduction of evolutionary computing optimization algorithms based on DE and GAs is presented here. These optimization solvers are exploited to obtain actual parameters by optimizing ESM model merit function (14). These algorithms may be exploited as a part of iterative learning control based hardware, if optimization of the decision variable is efficient and accurate then relatively lesser processing power as well as storage capacity is required that definitely assist to reduce the cost of the equipment's.

$\mathrm{DE}$ is the member of evolutionary search strategies developed by Storn and Price for continuo us domains optimization problems [74-75]. DE is one of best population based technique having 
benefits of simplicity, effortless implementation; speedy convergence and robustness for finding solution of real valued parameters. DE employs mutation as a search technique and selection is used to direct the prospective search toward the feasible region. DE has been broadly utilized to solve real world problems in engineering domain such as simultaneous transit network design [76], facial expression recognition systems [77], self-paced stacked denoising autoencoders [78], optimal reactive power dispatch systems [79], optimized watermarking [80] and image processing [81]. 

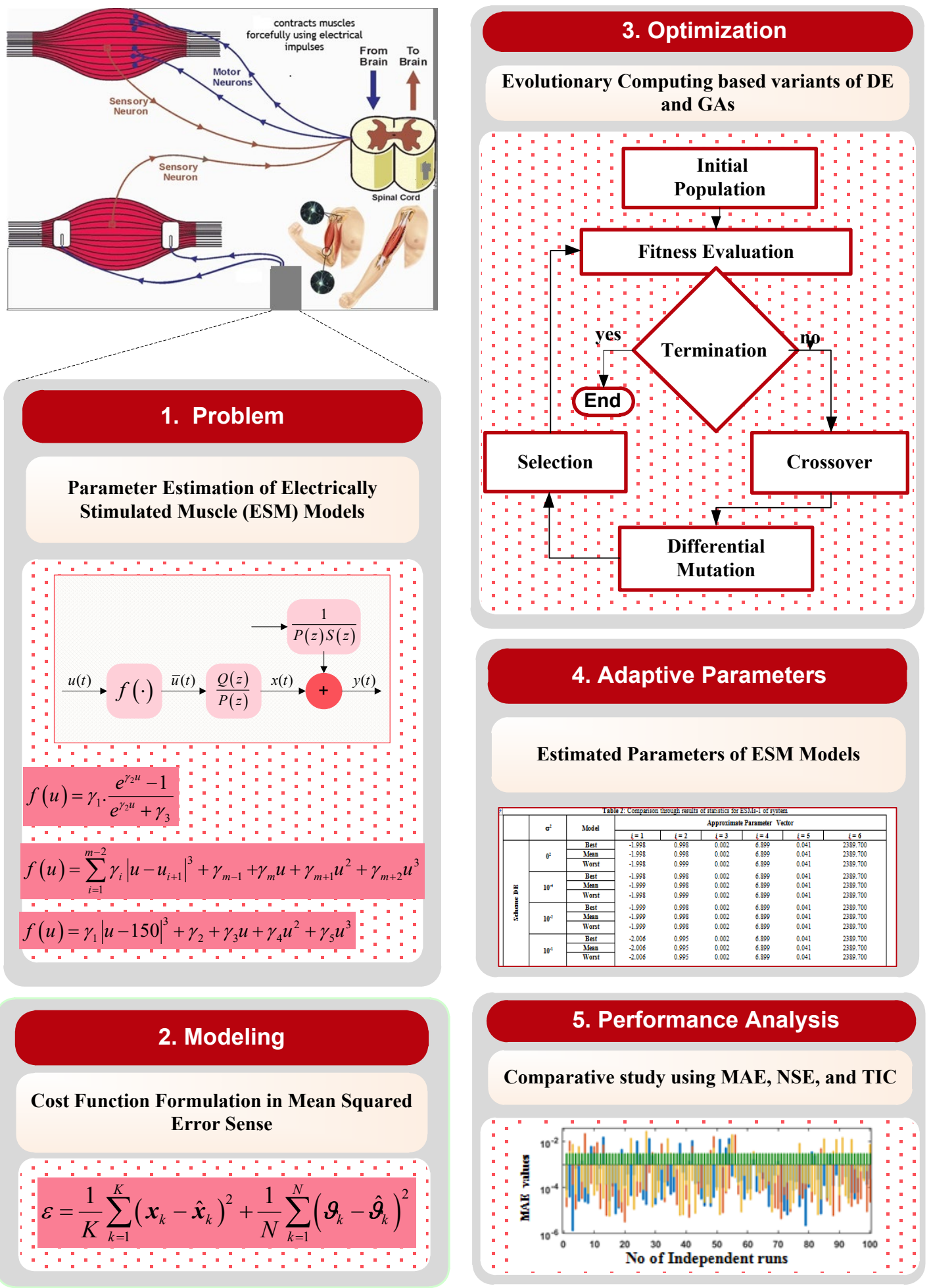

\section{Performance Analysis}

Comparative study using MAE, NSE, and TIC

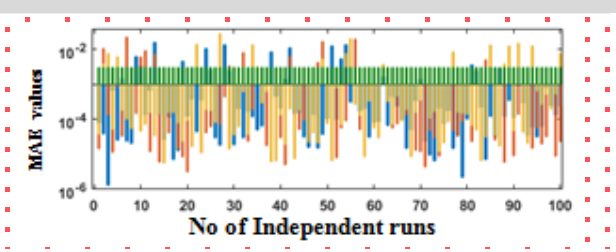

Fig. 1: Schematic diagram of proposed methodologies

Genetic algorithms is one of the best global search procedure belonging to the class of evolutio nary computing heuristics. GAs are introduced by Holland [82-83], on the basis of simple mathematical modeling of genetics. Normally, GAs operate with three fundamental operators used in reproduction of the population at each generation based on selection, mutation and crossover. GAs 
is widely employed for different optimization problems of engineering and applied sciences including sentiment analysis of microblogs [84], viable intrusion detection system design [85], disassembly sequence planning problems [86], transit network design problems [87], feature selection for credit rating problems [88] and optimization of Sisko fluid flow based heat transfer models [89].

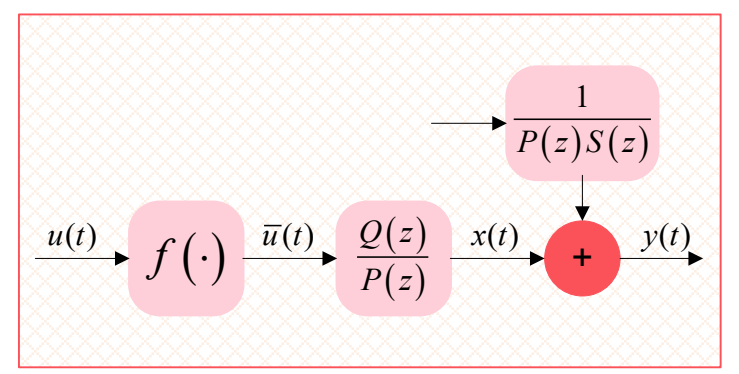

Fig. 2. The Hammerstein control autoregressive block structure

Effectiveness of these meta-heuristic algorithms based on DE and GAs motivates authors to exploit the strength of evolutionary optimization procedures in obtaining the optimization variables of ESM models. Besides that, the procedure of PSO, PS and SA with settings of the parameter as given in the references [90-92] are also implemented for finding the decision variables of ESM model. Generic flow diagram of DE is illustrated in Fig. 3 while stepwise procedure is described in Table. 1

Table 1: Pseudocode for DE algorithm for identification problem of ESM models

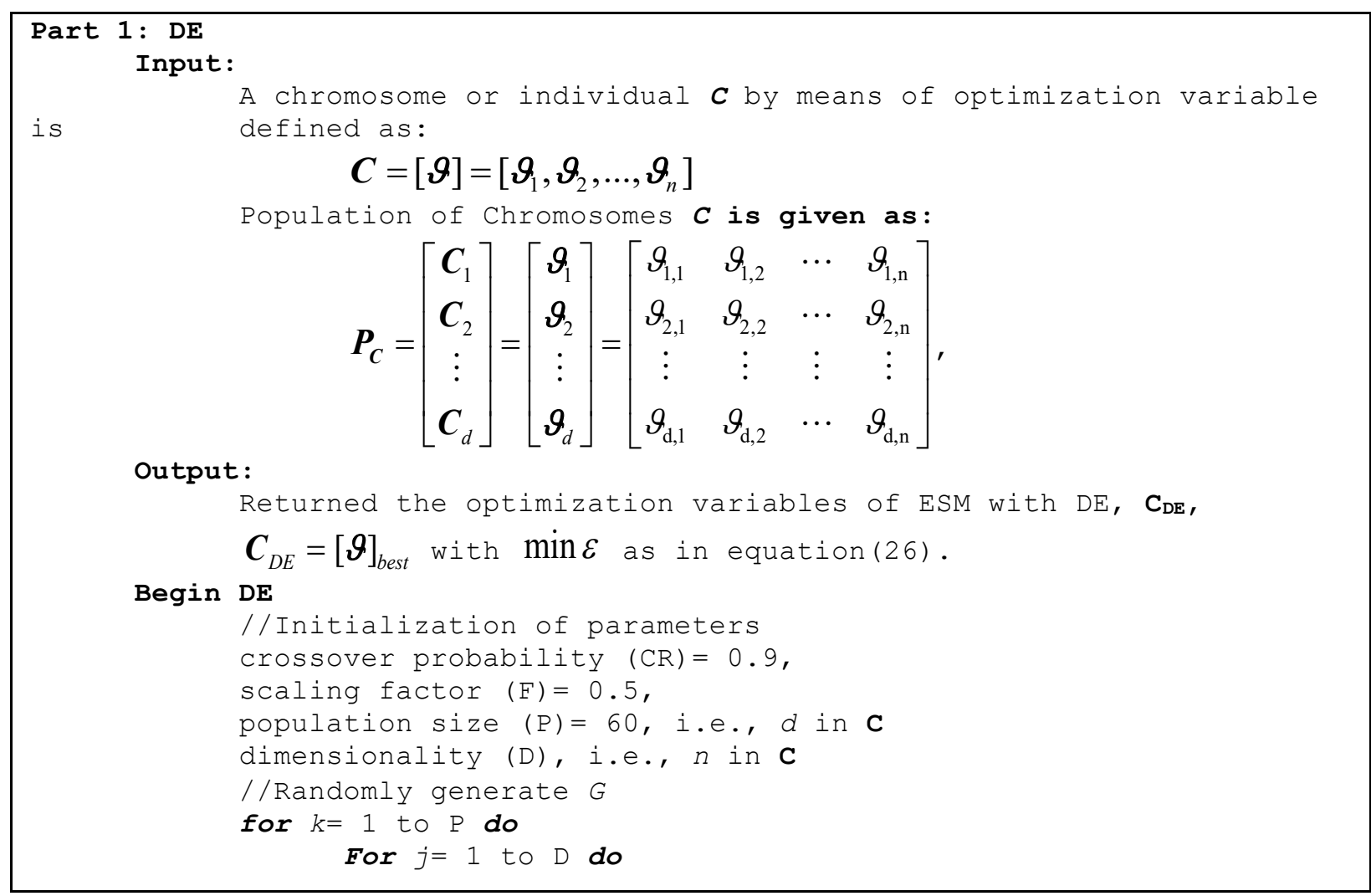




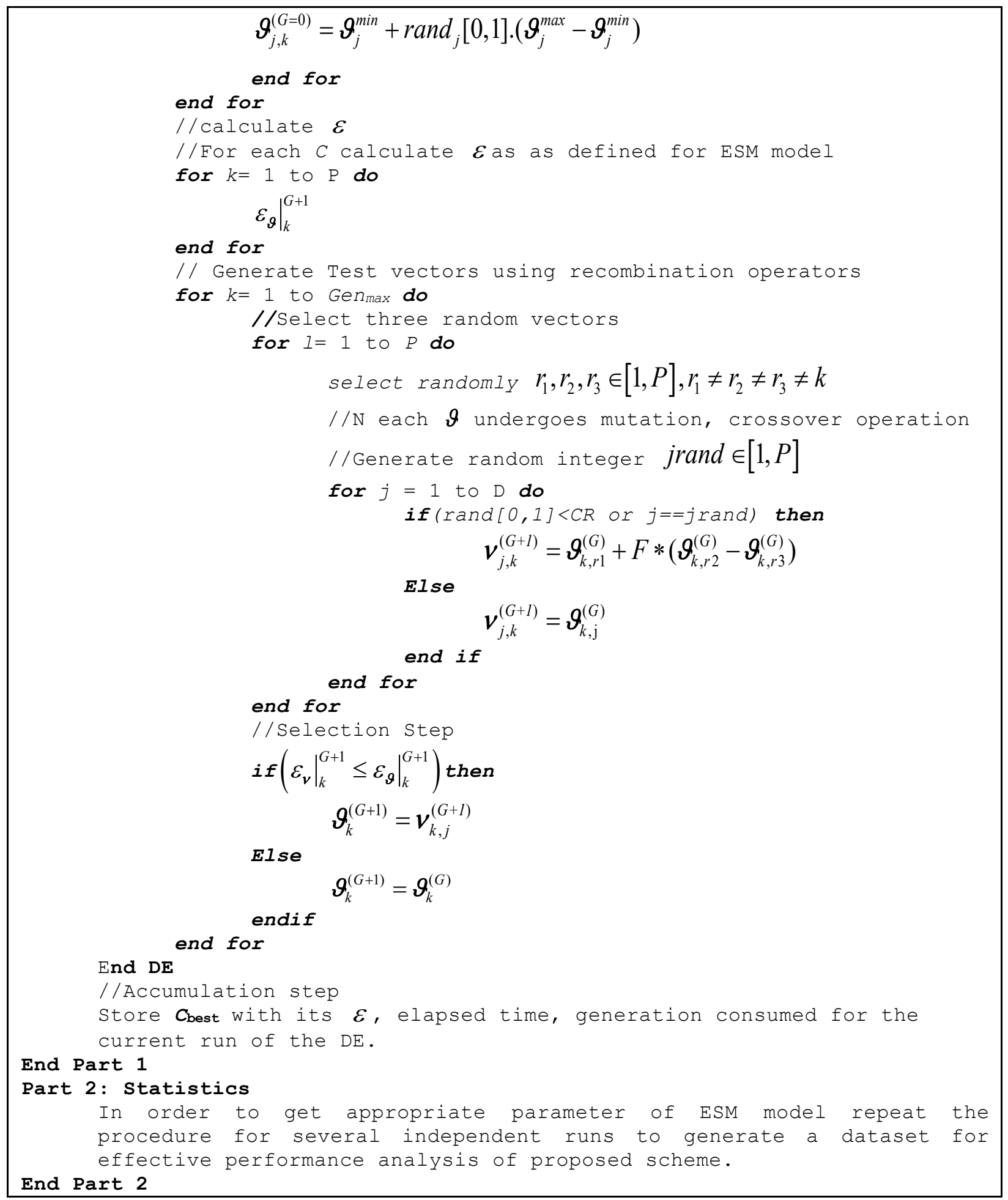




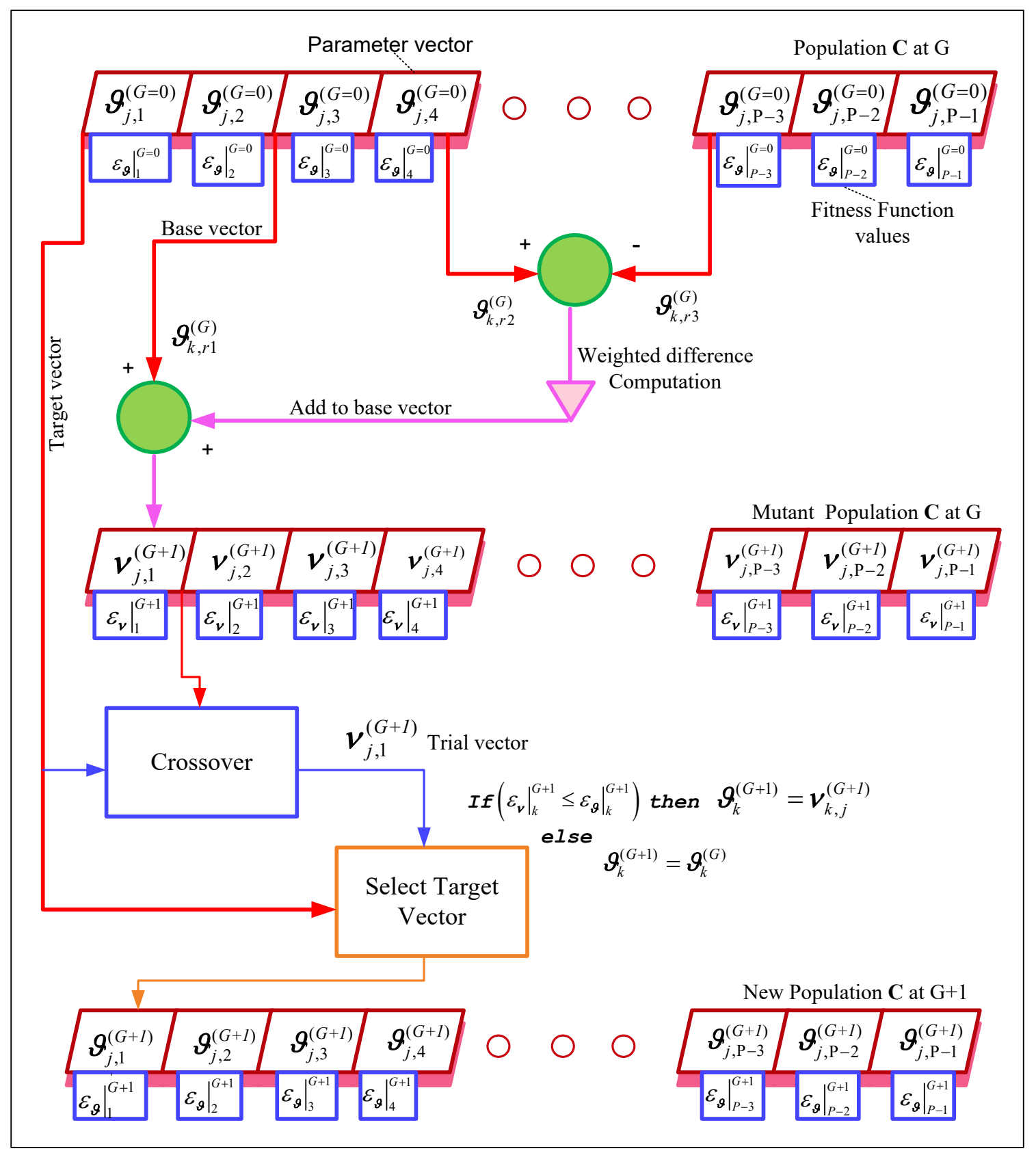

Fig. 3: Workflow diagram of DE for ESM model estimation of parameters

\section{Performance Operators}

The performance indices for $\vartheta$ in terms of mean absolute error $\left(M A E_{\vartheta}\right)$, normalizing error function, $\delta_{\vartheta}$, Thiel's inequality coefficient, $\left(T I C_{\vartheta}\right)$ and Nash Sutcliffe efficiency $\left(N S E_{\vartheta}\right)$ are utilized to access the designed schemes for parameter estimation of ESM models. The mathematical relations of all these operators are written as follows.

The $M A E_{\vartheta}$ is defined as: 


$$
M A E_{\vartheta}=\frac{1}{n} \sum_{i=1}^{n}\left|\vartheta_{i}-\hat{\vartheta}_{i}\right|,
$$

here $\vartheta_{i}$ is the $i^{\text {th }}$ element of actual parameter vector $\vartheta_{i}$.

Thiel's Inequality Coefficient $\left(T I C_{\vartheta}\right)$ is mathematically written as:

$$
T I C_{\vartheta}=\frac{\sqrt{\frac{1}{n} \sum_{i=1}^{n}\left(\vartheta_{i}-\hat{\vartheta}_{i}\right)^{2}}}{\left(\sqrt{\frac{1}{n} \sum_{i=1}^{n} \vartheta_{i}^{2}}+\sqrt{\frac{1}{n} \sum_{i=1}^{n} \hat{\vartheta}_{i}^{2}}\right)}
$$

Normalize error function (NEF) in term of mean weigh deviation

$$
\delta_{\vartheta}=\frac{\|\hat{\vartheta}-\vartheta\|}{\|\vartheta\|}
$$

where \|\| is the standard $L^{2}$ norm.

Nash Sutcliffe efficiency $\left(N S E_{\vartheta}\right)$ is formulated as:

$$
N S E_{\vartheta}=1-\frac{\sum_{i=1}^{n}\left(\vartheta_{i}-\hat{\vartheta}_{i}\right)^{2}}{\sum_{i=1}^{n}\left(\vartheta_{i}-\bar{\vartheta}_{i}\right)^{2}},
$$

and its related error term $\mathrm{ENSE}_{\vartheta}$ is $1-N S E_{\vartheta}$.

Similarly, the global version of $M A E_{\vartheta}\left(G M A E_{\vartheta}\right)$ is formalized as:

$$
G M A E_{\vartheta}=\frac{1}{I_{E}} \sum_{e=1}^{I_{E}}\left(M A E_{\vartheta}\right)_{e}=\frac{1}{I_{E}} \sum_{e=1}^{I_{E}}\left(\frac{1}{n} \sum_{i=1}^{n}\left|\vartheta_{i}-\hat{\vartheta}_{i}\right|\right)_{e},
$$

here, symbol $I_{E}$ denotes the total number of independent executions.

Global $T I C_{\vartheta}\left(G_{T I C \vartheta}\right)$ is formulated as:

$$
G_{T I C \vartheta}=\frac{1}{I_{E}} \sum_{e=1}^{I_{E}}\left(T I C_{\vartheta}\right)_{e}=\frac{1}{I_{E}} \sum_{e=1}^{I_{E}}\left(\frac{\sqrt{\frac{1}{n} \sum_{i=1}^{n}\left(\vartheta_{i}-\hat{\vartheta}_{i}\right)^{2}}}{\left(\sqrt{\frac{1}{n} \sum_{i=1}^{n} \vartheta_{i}^{2}}+\sqrt{\frac{1}{n} \sum_{i=1}^{n} \hat{\vartheta}_{i}^{2}}\right)}\right)_{e}
$$


Accordingly, global version of Nash Sutcliffe efficiency $\left(N S E_{\vartheta}\right)$ is formulated as:

$$
G_{N S E \vartheta}=\frac{1}{I_{E}} \sum_{e=1}^{I_{E}}\left(N S E_{\vartheta}\right)_{e}=\frac{1}{I_{E}} \sum_{e=1}^{I_{E}}\left(1-\frac{\sum_{i=1}^{n}\left(\vartheta_{i}-\hat{\vartheta}_{i}\right)^{2}}{\sum_{i=1}^{n}\left(\vartheta_{i}-\bar{\vartheta}_{i}\right)^{2}}\right)_{e} .
$$

Additionally, mean fitness $\bar{\varepsilon}_{\vartheta}$ in terms of total number of executions $I_{E}$ is formulated as:

$$
\bar{\varepsilon}_{\vartheta}=\frac{1}{I_{E}} \sum_{e=1}^{I_{E}}\left(\varepsilon_{\vartheta}\right)_{e}=\frac{1}{I_{E}} \sum_{e=1}^{I_{E}}\left(\frac{1}{K} \sum_{k=1}^{K}\left(x_{k}-\hat{x}_{k}\right)^{2}+\frac{1}{N} \sum_{k=1}^{N}\left(\vartheta_{k}-\hat{\vartheta}_{k}\right)^{2}\right)_{e}
$$

In the ideal case, values of the performance indices must be zero.

\section{Experimentation and Results}

Results for parameter estimation for three ESM models are presented through DE and GAs based evolutionary computational heuristics. In all three ESM systems, the performance of both optimization solvers is evaluated and presented here for a noiseless scenario as well as three noisy cases with different variances, i.e., $\sigma^{2}=0.001^{2}, 0.01^{2}$, and $0.1^{2}$.

ESM-I: ESM model with sigmoid Kernel based input nonlinearity: The parameters estimation of ESM-1 based on available dataset reported in [11] as followings:

$$
\begin{aligned}
& x(t)=\frac{P(z)}{Q(z)} g(u(t))+\frac{1}{Q(z)} n(t), \\
& Q(z)=1+q_{1} z^{-1}+q_{2} z^{-2}=1-1.9985 z^{-1}+0.9985 z^{-2}, \\
& P(z)=p_{1} z^{-1}=0.0022 z^{-1}, \\
& f(u(t))=\gamma_{1} \cdot \frac{e^{\gamma / 2 u(t)}-1}{e^{\gamma / u^{u}(t)}+\gamma_{3}}=6.8994 \cdot \frac{e^{0.0410 u(t)}-1}{e^{0.0410 u(t)}+2389.70}, \\
& x(t)=-[-1.9985 x(t-1)+0.9985 x(t-2)] \\
& \quad+0.0022\left[6.8994 \times \frac{e^{0.0410 u(t-1)}-1}{e^{0.0410 u(t-1)}+2389.7}\right]+n(t), \\
& \vartheta=\left[q_{1}, q_{2}, p_{1}, \gamma_{1}, \gamma_{2}, \gamma_{3}\right], \\
& \vartheta=[-1.9985,0.9985,0.0022,6.8994,0.0410,2389.70] .
\end{aligned}
$$

The fitness evaluation function for ESM-I is constructed accordingly to equation (26) by taking $K$ $=20$ and $N=6$ as:

$$
\varepsilon=\frac{1}{20} \sum_{k=1}^{20}\left(x_{k}-\hat{x}_{k}\right)^{2}+\frac{1}{6} \sum_{k=1}^{6}\left(\vartheta_{k}-\hat{\vartheta}_{k}\right)^{2}
$$


ESM-II: ESM model with polynomial $g(u(t))$ : The parameters estimation of ESM-II system with polynomial kernel $g(u(t))$ based on available dataset reported in [11] as followings:

$$
\begin{aligned}
& x(t)=\frac{P(z)}{Q(z)} g(u(t))+\frac{1}{Q(z)} n(t), \\
& Q(z)=1+q_{1} z^{-1}+q_{2} z^{-2}=1-z^{-1}+0.8 z^{-2}, \\
& P(z)=p_{1} z^{-1}+p_{2} z^{-2}=z^{-1}+0.6 z^{-2} \text {, } \\
& f(u(t))=\gamma_{1} u(t)+\gamma_{2} u^{2}(t)+\gamma_{3} u^{3}(t)=2.8 u(t)-4.8 u^{2}(t)+5.7 u^{3}(t), \\
& x(t)=-[-1.00 x(t-1)+0.8 x(t-2)]+[(1)(2.8)] u(t-1) \\
& +[(1)(-4.8)] u^{2}(t-1)+[(1)(5.7)] u^{3}(t-1)+[(0.6)(2.8)] u(t-2) \\
& +[(0.6)(-4.8)] u^{2}(t-2)+[(0.6)(5.7)] u^{3}(t-2)+n(t), \\
& \vartheta=\left[q_{1}, q_{2}, p_{1}, p_{2}, \gamma_{1}, \gamma_{2}, \gamma_{3}\right] \text {, } \\
& \vartheta=[-1.00,0.80,1.00,0.60,2.80,-4.80,5.70] \text {. }
\end{aligned}
$$

The fitness function constructed on similar pattern used for ESM-I and given for the said case as:

$$
\varepsilon=\frac{1}{20} \sum_{k=1}^{20}\left(x\left(t_{k}\right)-\hat{x}\left(t_{k}\right)\right)^{2}+\frac{1}{7} \sum_{k=1}^{7}\left(\vartheta_{k}-\hat{\vartheta}_{k}\right)^{2} .
$$

ESM-III: ESM model with cubic-spline $g(u(t))$ : The parameters estimation of EMS-II model with cubic-spline kernel $g(u(t))$ have one know at 150 based on available dataset reported in [11] as followings:

$$
\begin{aligned}
& x(t)=\frac{P(z)}{Q(z)} g(u(t))+\frac{1}{Q(z)} n(t), \\
& Q(z)=1+q_{1} z^{-1}+q_{2} z^{-2}=1-1.094 z^{-1}+0.109 z^{-2} \\
& P(z)=p_{1} z^{-1}+p_{2} z^{-2}=z^{-1}+0.249 z^{-2}, \\
& f(u(t))=\gamma_{1}+\gamma_{2} u(t)+\gamma_{3} u^{2}(t)+\gamma_{4} u^{3}(t)+\gamma_{5}\left|u^{3}(t)-150\right|, \\
& f(u(t))=-0.028+1.90 \times 10^{-3} u(t)-7.83 \times 10^{-6} u^{2}(t) \\
& \quad+1.78 \times 10^{-8} u^{3}(t)+2.36 \times 10^{-8}\left|u^{3}(t)-150\right|
\end{aligned}
$$




$$
\begin{aligned}
& x(t)=- {[-1.094 x(t-1)+0.109 x(t-2)]+[(1)(-0.028)] } \\
&+ {\left[(1)\left(1.90 \times 10^{-3}\right)\right] u(t-1)+\left[(1)\left(-7.83 \times 10^{-6}\right)\right] u^{2}(t-1) } \\
&+\left[(1)\left(1.78 \times 10^{-8}\right)\right] u^{3}(t-1)+\left[(1)\left(2.36 \times 10^{-8}\right)\right]\left|u^{3}(t-1)-150\right| \\
&+[(0.249)(-0.028)]+\left[(0.249)\left(1.90 \times 10^{-3}\right)\right] u(t-1) \\
&+\left[(0.249)\left(-7.83 \times 10^{-6}\right)\right] u^{2}(t-1)+\left[(0.249)\left(1.78 \times 10^{-8}\right)\right] u^{3}(t-1) \\
&+\left[(0.249)\left(2.36 \times 10^{-8}\right)\right]\left|u^{3}(t-1)-150\right|+n(t), \\
& \vartheta=\left[q_{1}, q_{2}, p_{1}, p_{2}, \gamma_{1}, \gamma_{2}, \gamma_{3}, \gamma_{4}, \gamma_{5}\right], \\
& \vartheta=\left[\begin{array}{l}
-1.094,0.109,1.00,-0.249,-0.028, \\
\left.1.90 \times 10^{-3},-7.83 \times 10^{-6}, 1.78 \times 10^{-8}, 2.36 \times 10^{-8}\right] .
\end{array}\right.
\end{aligned}
$$

The fitness function for ESM-III is formulated for $\mathrm{K}=20$ and $\mathrm{N}=9$ as:

$$
\varepsilon=\frac{1}{20} \sum_{k=1}^{20}\left(x\left(t_{k}\right)-\hat{x}\left(t_{k}\right)\right)^{2}+\frac{1}{9} \sum_{k=1}^{9}\left(\vartheta_{k}-\hat{\vartheta}_{k}\right)^{2}
$$

The designed heuristic techniques based on DE and GAs have been performed for 100 independent trails to optimize the fitness functions for all three ESM models in case of noiseless and noisy scenarios. The results of DE and GAs on the basis of learning curves, i.e., iterative convergence plots, are presented in the graphical form in Fig. 4, for both ESM-I and ESM-II, in case of four noise variances $\sigma^{2}=0.001^{2}, 0.01^{2}, 0.1^{2}$ and 0 . In all these plots, it is observed that for each noise scenario both DE and GAs are convergent, but DE attains better fitness.

To access the level of the accuracy attained by the algorithms, magnitudes of absolute error (AE) are computed for each case of all three ESM models for noise levels $\sigma^{2}=0.001^{2}, 0.01^{2}, 0.1^{2}$ and 0 . The results are presented in Figs. 5-6 for the best trials of DE and GAs based on minimum fitness, respectively. The magnitudes of $\mathrm{AE}$ are found close to $10^{-09}$ to $10^{-12}, 10^{-09}$ to $10^{-12}, 10^{-09}$ to $10^{-11}$ and $10^{-04}$ to $10^{-11}$ for noise variances $\sigma^{2}=0.001^{2}, 0.01^{2}, 0.1^{2}$ and 0 , respectively, for $\mathrm{DE}$, while for GAs respective $\mathrm{AE}$ are found close to $10^{-08}$ to $10^{-09}, 10^{-04}$ to $10^{-08}, 10^{-05}$ to $10^{-11}$ and $10^{-04}$ to $10^{-7}$. It is also noticed that the designed scheme is convergent for all three ESM systems, but a small degradation in the accuracy of the techniques is observed with an increase in the nose levels, for both algorithms. 


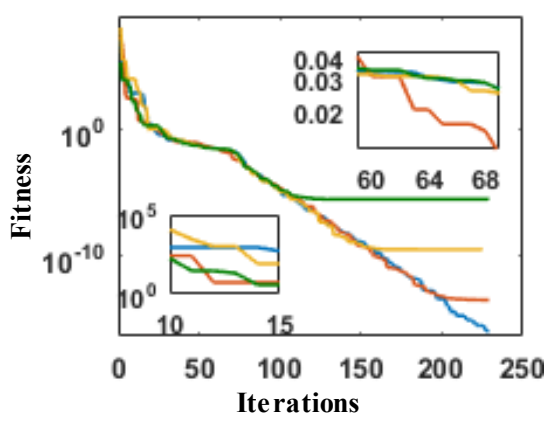

(a) DE learning curves for ESM-I

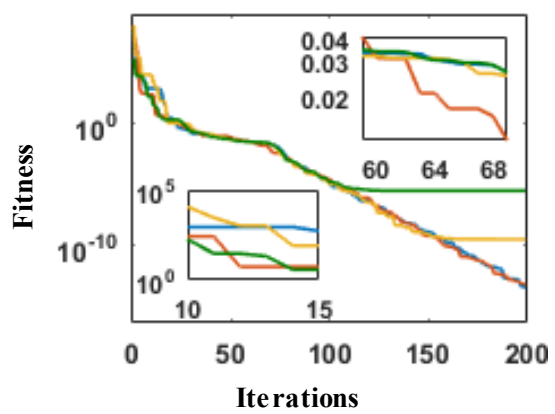

(d) GAs learning curves for ESM-II

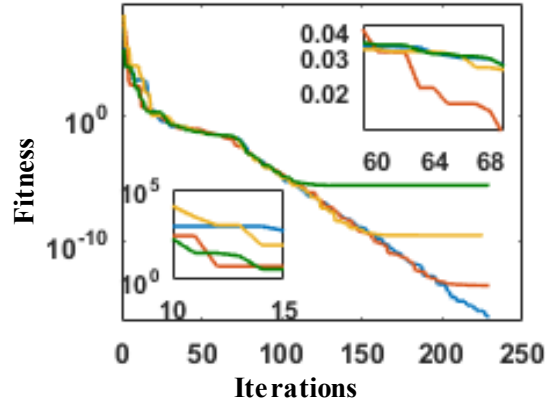

(b) GAs learning curves for ESM-I

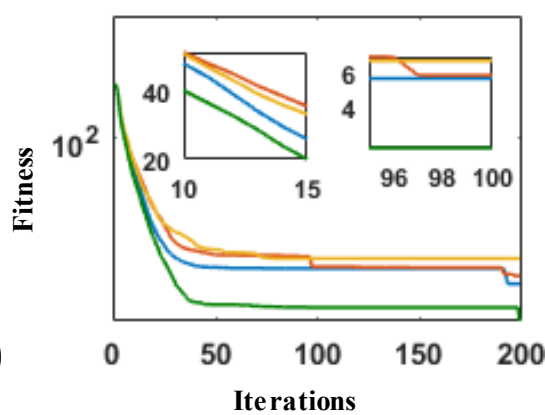

(e) DE learning curves for ESM-III

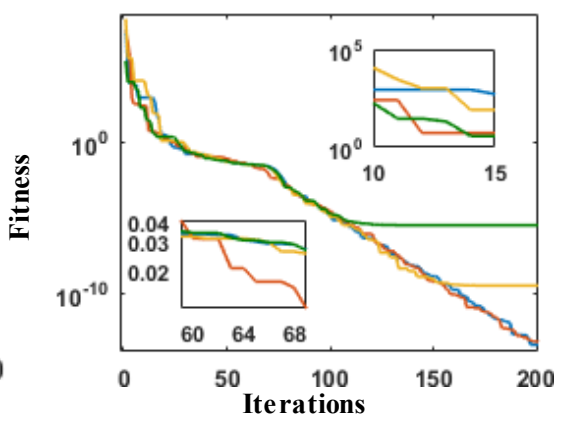

(c) DE learning curves for ESM-II

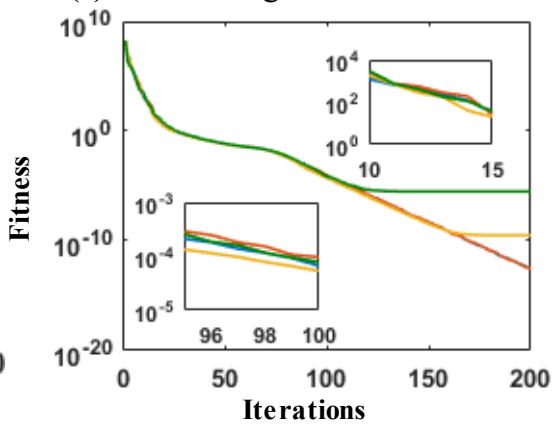

(f) GAs learning curves for ESM-III

$$
\square \sigma^{2}=0^{2}-\sigma^{2}=0.001^{2}-\sigma^{2}=0.01^{2}-\sigma^{2}=0.1^{2}
$$

Fig. 4. Results for convergence analysis of merit function of ESM-I, II and III

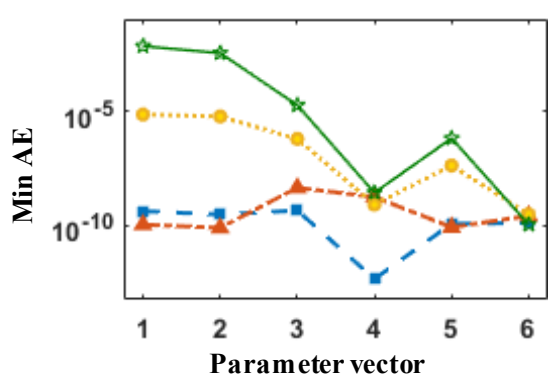

(a) DE based AE for ESM-I

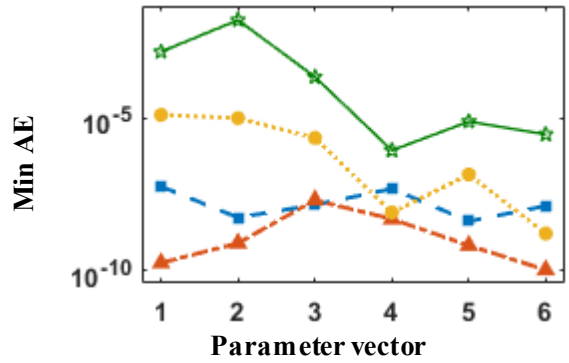

(c) GAs based AE for ESM-I

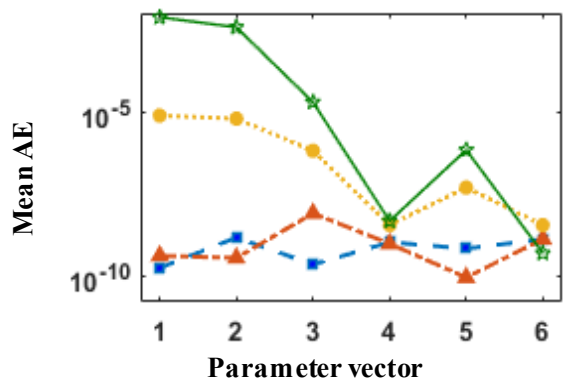

(b) DE based AE for ESM-I

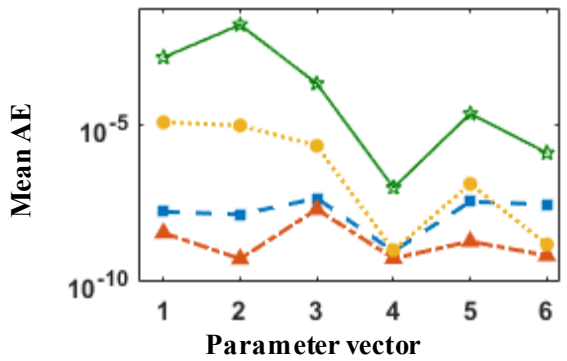

(d) GAs based AE for ESM-I

$$
-=\sigma^{2}=0^{2}-\Delta-\sigma^{2}=0.001^{2} \cdots 0 \cdots \sigma^{2}=0.01^{2} \rightarrow \sigma^{2}=0.1^{2}
$$

Fig. 5. Comparison of absolute error for ESM-1 systems with different noise levels 


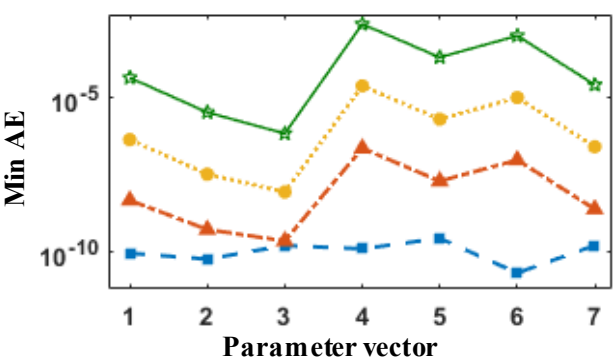

(a) DE based AE for ESM-II

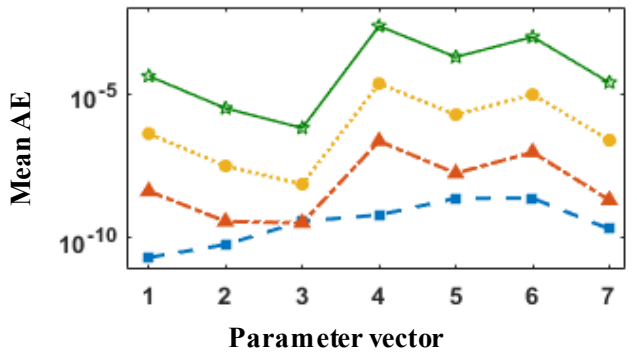

(b) DE based AE for ESM-II

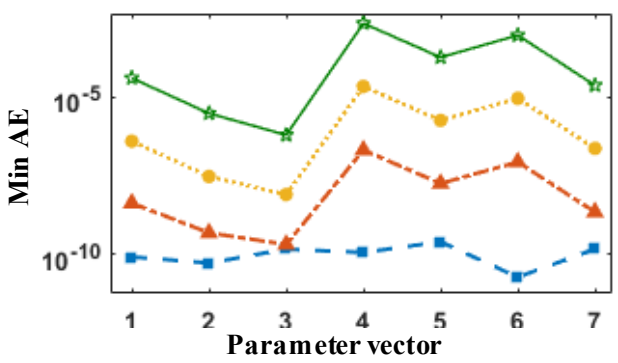

(c) GAs based AE for ESM-II

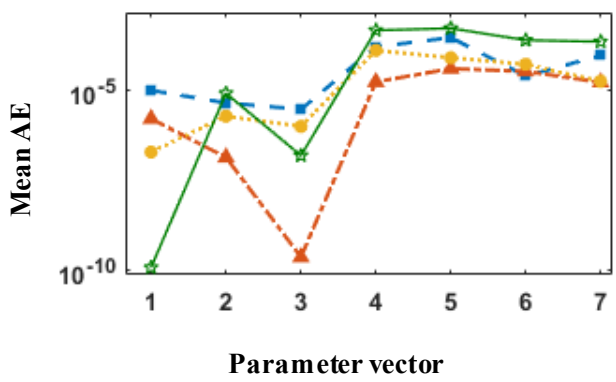

(d) GAs based AE for ESM-II

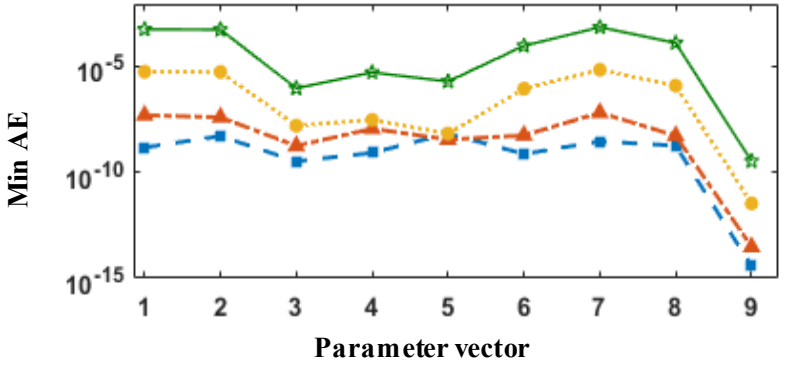

(e) DE based AE for ESM-III

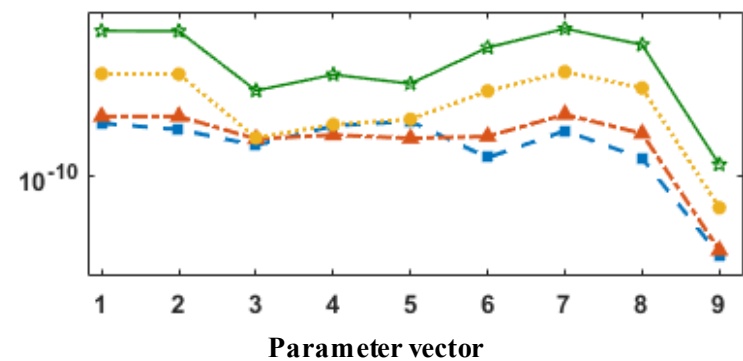

(f) DE based AE for ESM-III

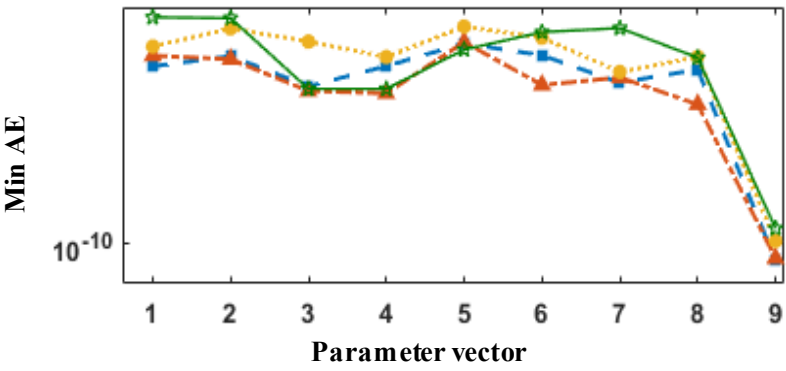

(g) GAs based AE for ESM-III

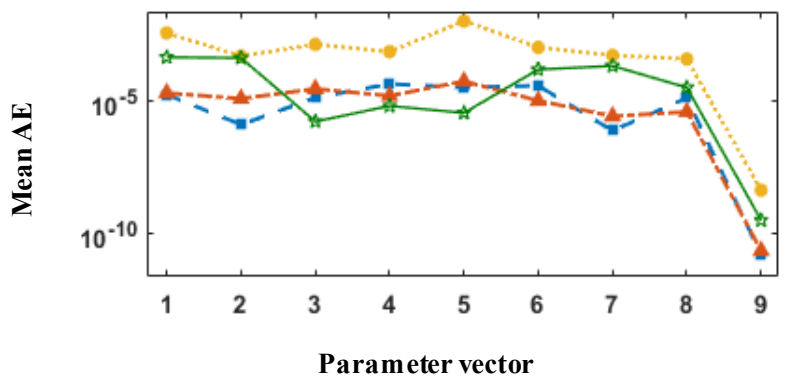

(h) GAs based AE for ESM-III

$$
-\sigma^{2}=0^{2}-\triangle-\sigma^{2}=0.001^{2} \cdots 0 \cdots \sigma^{2}=0.01^{2} \leadsto \sigma^{2}=0.1^{2}
$$

Fig. 6. Comparison on AE for ESM-II and III systems with different noise levels

The evaluation of for both DE and GAs is performed through performance indices based on accuracy criterion of fitness $\varepsilon$, normalized error function $\delta_{\vartheta}, M A E_{\vartheta}, N S E_{\vartheta}$, and $T I C_{\vartheta}$, as described mathematically in the last section. Results on the basis of best fitness are presented in Table 2 along with the complexity operators in terms of time, cycles and function counts. It can be seen that the MWD, $\delta$ lie around $10^{-03}$ to $10^{-08}, 10^{-03}$ to $10^{-08}$ in case of ESM-I for both DE and GAs, respectively, while for ESM-II values are found close to $10^{-03}$, whereas in case of ESM-III respective values of DE and GAs ranges between $10^{-03}$ to $10^{-05}$, and $10^{-03}$ to $10^{-09}$. In general, the 
significantly near-optimal gauges of these performance operators are obtained which ascertains the consistent accuracy of the proposed schemes, however DE is placed at the higher ranks by achieving relatively lower values of the indices than that of GAs.

Table 2: Comparison of the performance on basis of best runs of both algorithms for each variant of ESM models

\begin{tabular}{|c|c|c|c|c|c|c|c|c|c|c|}
\hline \multirow{2}{*}{ Solver } & \multirow{2}{*}{ ESM } & \multirow{2}{*}{$\sigma^{2}$} & \multicolumn{5}{|c|}{ Accuracy } & \multicolumn{3}{|c|}{ Complexity } \\
\hline & & & $\varepsilon$ & $\delta$ & MAE & NSE & TIC & Time & Gens & FC \\
\hline \multirow{12}{*}{ DE } & \multirow{4}{*}{ I } & $\overline{0}$ & $8.86 \mathrm{E}-20$ & $3.05 \mathrm{E}-13$ & $2.43 \mathrm{E}-10$ & $0.00 \mathrm{E}+00$ & $4.92 \mathrm{E}-13$ & 7.81 & 192 & 34751 \\
\hline & & $10^{-3}$ & 3.34E-12 & $6.83 \mathrm{E}-13$ & $1.16 \mathrm{E}-09$ & $0.00 \mathrm{E}+00$ & $1.72 \mathrm{E}-12$ & 7.64 & 217 & 39276 \\
\hline & & $10^{-2}$ & 3.34E-08 & $5.67 \mathrm{E}-13$ & 2.40E-06 & $0.00 \mathrm{E}+00$ & 2.05E-09 & 7.47 & 208 & 37647 \\
\hline & & $10^{-1}$ & 1.83E-04 & $1.66 \mathrm{E}-12$ & $1.87 \mathrm{E}-03$ & 7.33E-11 & $1.75 \mathrm{E}-06$ & 7.79 & 215 & 38914 \\
\hline & \multirow{4}{*}{ II } & $\overline{0}$ & $4.56 \mathrm{E}-18$ & 1.64E-12 & $1.20 \mathrm{E}-10$ & $0.00 \mathrm{E}+00$ & $2.05 \mathrm{E}-10$ & 10.69 & 191 & 40300 \\
\hline & & $10^{-3}$ & $9.61 \mathrm{E}-14$ & 7.19E-13 & 4.63E-08 & 3.79E-14 & $1.41 \mathrm{E}-08$ & 9.69 & 176 & 37135 \\
\hline & & $10^{-2}$ & $9.60 \mathrm{E}-10$ & 1.79E-12 & 4.83E-06 & $4.15 \mathrm{E}-10$ & $1.47 \mathrm{E}-06$ & 9.77 & 181 & 38190 \\
\hline & & $10^{-1}$ & $9.60 \mathrm{E}-06$ & 2.01E-12 & $1.87 \mathrm{E}-03$ & 4.15E-06 & 1.47E-04 & 9.64 & 179 & 37768 \\
\hline & \multirow{4}{*}{ III } & $\mathbf{0}$ & $1.26 \mathrm{E}-16$ & $6.23 \mathrm{E}-13$ & 2.14E-09 & $2.22 \mathrm{E}-16$ & 2.93E-09 & 19.12 & 236 & 63955 \\
\hline & & $10^{-3}$ & $3.14 \mathrm{E}-14$ & 7.97E-13 & $1.94 \mathrm{E}-08$ & 2.69E-14 & 2.82E-08 & 17.00 & 220 & 59619 \\
\hline & & $10^{-2}$ & $3.12 \mathrm{E}-10$ & $1.15 \mathrm{E}-12$ & 2.12E-06 & $3.73 \mathrm{E}-10$ & $3.32 \mathrm{E}-06$ & 16.78 & 220 & 59619 \\
\hline & & $10^{-1}$ & 3.12E-06 & $3.59 \mathrm{E}-13$ & $1.87 \mathrm{E}-03$ & $3.69 \mathrm{E}-06$ & $3.29 \mathrm{E}-04$ & 24.96 & 230 & 62329 \\
\hline \multirow{12}{*}{ GAs } & \multirow{4}{*}{ I } & $\mathbf{0}$ & $9.87 \mathrm{E}-16$ & $3.22 \mathrm{E}-11$ & 2.36E-08 & $0.00 \mathrm{E}+00$ & $1.48 \mathrm{E}-11$ & 10.37 & 300 & 60200 \\
\hline & & $10^{-3}$ & 7.57E-12 & $3.21 \mathrm{E}-11$ & 4.48E-09 & $0.00 \mathrm{E}+00$ & 4.48E-12 & 11.16 & 300 & 60200 \\
\hline & & $10^{-2}$ & 7.56E-08 & $3.04 \mathrm{E}-11$ & 4.19E-06 & $2.22 \mathrm{E}-16$ & 3.42E-09 & 9.72 & 300 & 60200 \\
\hline & & $10^{-1}$ & 4.01E-04 & $2.66 \mathrm{E}-11$ & $3.04 \mathrm{E}-03$ & $2.87 \mathrm{E}-10$ & 3.46E-06 & 10.64 & 300 & 60200 \\
\hline & \multirow{4}{*}{ II } & $\mathbf{0}$ & 8.90E-07 & 2.92E-11 & $1.18 \mathrm{E}-04$ & $1.42 \mathrm{E}-07$ & 2.00E-05 & 24.68 & 700 & 140200 \\
\hline & & $10^{-4}$ & $1.24 \mathrm{E}-05$ & 3.19E-11 & 2.41E-03 & 6.09E-05 & 5.63E-04 & 25.24 & 700 & 140200 \\
\hline & & $10^{-2}$ & $1.90 \mathrm{E}-06$ & 2.77E-11 & $1.90 \mathrm{E}-04$ & 8.03E-08 & 2.05E-05 & 24.88 & 700 & 140200 \\
\hline & & $10^{-1}$ & $1.30 \mathrm{E}-05$ & $2.88 \mathrm{E}-11$ & 3.04E-03 & $9.75 \mathrm{E}-07$ & 7.13E-05 & 23.96 & 700 & 140200 \\
\hline & \multirow{4}{*}{ III } & $\mathbf{0}$ & $1.18 \mathrm{E}-09$ & 3.13E-11 & $2.05 \mathrm{E}-05$ & 2.13E-08 & 2.50E-05 & 20.64 & 500 & 100200 \\
\hline & & $10^{-4}$ & $1.22 \mathrm{E}-09$ & 2.61E-11 & $1.67 \mathrm{E}-05$ & $2.82 \mathrm{E}-09$ & $9.11 \mathrm{E}-06$ & 21.33 & 500 & 100200 \\
\hline & & $10^{-2}$ & $1.74 \mathrm{E}-08$ & $3.21 \mathrm{E}-11$ & 8.02E-05 & $2.13 \mathrm{E}-09$ & 7.92E-06 & 19.99 & 500 & 100200 \\
\hline & & $10^{-1}$ & $2.47 \mathrm{E}-06$ & $3.23 \mathrm{E}-11$ & $3.04 \mathrm{E}-03$ & $1.36 \mathrm{E}-06$ & $2.00 \mathrm{E}-04$ & 21.01 & 500 & 100200 \\
\hline
\end{tabular}

The reliability of the proposed scheme is examined through 100 independent runs in each noise scenario for all three examples of ESM models. The results in term fitness are presented in Fig. 7, while comparative analysis on the basis of performance indicators, i.e., $M A E_{\vartheta}, N S E_{\vartheta}$ and $T I C_{\vartheta}$, are shown graphically in Figs. 8-9 for ESM-I, II and III, respectively. The $T I C_{\vartheta}$ graphs for ESM-I for noise scenario $\sigma^{2}=0.001^{2}$ and $\sigma^{2}=0.01^{2}$ are illustrated graphically in Fig. 10, in the form of empirical cumulative distribution function plots for both DE and GAs. Additionally, the $M A E$ for ESM-I and II are presented using semi-logarithmic scale in order to interpret small variation and are given in subfigures $11(a-b)$ for DE and subfigures $11(c-d)$ for GAs. These results show that the magnitudes of MAE for ESM-I are in the range of $10^{-04}$ to $10^{-20}$, and $10^{-04}$ to $10^{-16}$ in case of $\mathrm{DE}$ and GAs, respectively. While for ESM-II values lie are in the range of $10^{-06}$ to $10^{-18}$, and $10^{-05}$ to $10^{-07}$ for DE and GAs, respectively. Further accuracy evaluation of the proposed strategies is conducted through the plots of histogram and results on the basis of NSEs for ESM-I and II are presented in subfigures $11(e-h)$ and subfigures. $11(\mathrm{i}-l)$ respectively. Furthermore, analysis on the basis of TIC for the two problems are presnted as stack bar plots in subfigures $11(m-n)$ for DE and GAs, respectively. Moreover, ESM-III illustrations are provided in Fig. 12. All these graphs 
validate the consistency of both the schemes designed for the parameter estimation problem of ESM systems for all four noise scenarios; Additional graph of comparison of both the studies is also provided in the form of bar plots and results are provided in Fig. 12 these results verify that DE outperforms GAs.

The detailed evaluation of the accuracy of the algorithm is carried out by statistical performance measures of mean, best, and worst magnitudes of fitness for $\sigma^{2}=0.001^{2}, 0.01^{2}$, and $0.1^{2}$ as given in Table 3 for ESM-I along with the desired parameters. Similarly, the results for ESM-II and ESM-III are provided in Tables 4 and 5, respectively. It is observed that with the increase in noise level $\sigma^{2}=0.001^{2}$ to $0.1^{2}$ degradation in the performance is noticed in case of both DE and GAs, however, both the algorithms are significantly applicable for finding the optimal ESM parameters with reasonable accuracy.

Table 3: Results of statistical operators for variants of ESM-I system

\begin{tabular}{|c|c|c|c|c|c|c|c|c|}
\hline \multirow{2}{*}{ Solver } & \multirow{2}{*}{$\sigma^{2}$} & \multirow{2}{*}{ Model } & \multicolumn{6}{|c|}{ Approxi mate Parameter Vector } \\
\hline & & & $i=1$ & $i=\mathbf{2}$ & $i=\mathbf{3}$ & $i=4$ & $i=5$ & $i=6$ \\
\hline \multirow{12}{*}{ DE } & \multirow{3}{*}{$\mathbf{0}^{2}$} & Best & -1.998 & 0.998 & 0.002 & 6.899 & 0.041 & 2389.700 \\
\hline & & Mean & -1.998 & 0.998 & 0.002 & 6.899 & 0.041 & 2389.700 \\
\hline & & Worst & -1.998 & 0.999 & 0.002 & 6.899 & 0.041 & 2389.700 \\
\hline & \multirow{3}{*}{$10^{-4}$} & Best & -1.998 & 0.998 & 0.002 & 6.899 & 0.041 & 2389.700 \\
\hline & & Mean & -1.999 & 0.998 & 0.002 & 6.899 & 0.041 & 2389.700 \\
\hline & & Worst & -1.998 & 0.999 & 0.002 & 6.899 & 0.041 & 2389.700 \\
\hline & \multirow{3}{*}{$10^{-2}$} & Best & -1.999 & 0.998 & 0.002 & 6.899 & 0.041 & 2389.700 \\
\hline & & Mean & -1.999 & 0.998 & 0.002 & 6.899 & 0.041 & 2389.700 \\
\hline & & Worst & -1.999 & 0.998 & 0.002 & 6.899 & 0.041 & 2389.700 \\
\hline & \multirow{3}{*}{$10^{-1}$} & Best & -2.006 & 0.995 & 0.002 & 6.899 & 0.041 & 2389.700 \\
\hline & & Mean & -2.006 & 0.995 & 0.002 & 6.899 & 0.041 & 2389.700 \\
\hline & & Worst & -2.006 & 0.995 & 0.002 & 6.899 & 0.041 & 2389.700 \\
\hline \multirow{12}{*}{ GAs } & \multirow{3}{*}{$0^{2}$} & Best & -1.998 & 0.999 & 0.002 & 6.899 & 0.041 & 2389.700 \\
\hline & & Mean & -1.998 & 0.999 & 0.002 & 6.899 & 0.041 & 2389.700 \\
\hline & & Worst & -1.998 & 0.998 & 0.002 & 6.899 & 0.041 & 2389.700 \\
\hline & \multirow{3}{*}{$10^{-4}$} & Best & -1.998 & 0.999 & 0.002 & 6.899 & 0.041 & 2389.700 \\
\hline & & Mean & -1.998 & 0.999 & 0.002 & 6.899 & 0.041 & 2389.700 \\
\hline & & Worst & -1.998 & 0.998 & 0.002 & 6.899 & 0.041 & 2389.700 \\
\hline & \multirow{3}{*}{$10^{-2}$} & Best & -1.999 & 0.998 & 0.002 & 6.899 & 0.041 & 2389.700 \\
\hline & & Mean & -1.999 & 0.998 & 0.002 & 6.899 & 0.041 & 2389.700 \\
\hline & & Worst & -1.999 & 0.998 & 0.002 & 6.899 & 0.041 & 2389.700 \\
\hline & \multirow{3}{*}{$10^{-1}$} & Best & -2.000 & 0.982 & 0.002 & 6.899 & 0.041 & 2389.700 \\
\hline & & Mean & -2.000 & 0.982 & 0.002 & 6.899 & 0.041 & 2389.700 \\
\hline & & Worst & -2.000 & 0.982 & 0.002 & 6.899 & 0.041 & 2389.700 \\
\hline \multicolumn{2}{|c|}{ True Parameter } & tor & -1.998 & 0.998 & 0.002 & 6.899 & 0.041 & 2389.700 \\
\hline
\end{tabular}



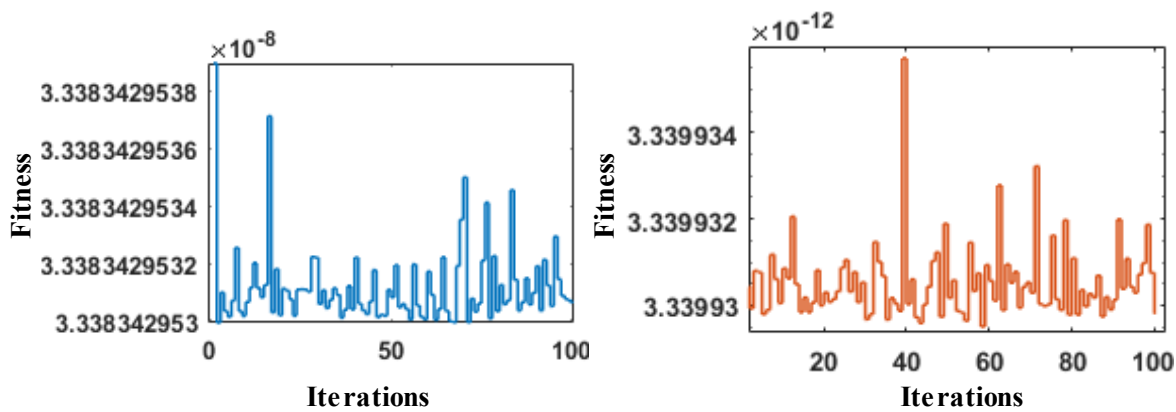

(a) $\sigma^{2}=0.00^{2}$

(b) $\sigma^{2}=0.001^{2}$
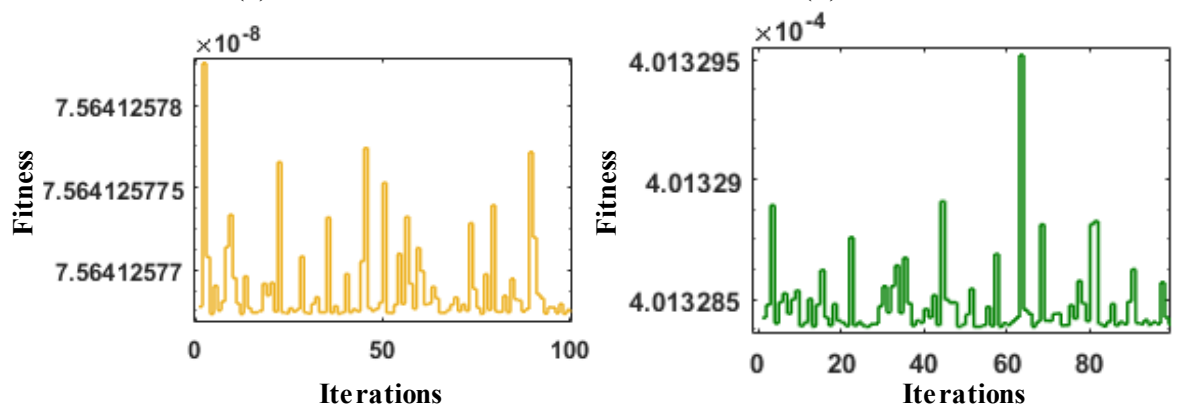

(c) $\sigma^{2}=0.01^{2}$

(d) $\sigma^{2}=0.1^{2}$

Fig. 7 Fitness plots for ESM-1 system with different noise levels using DE

Table 4: Results of statistical operators for variants of ESM-II system

\begin{tabular}{|c|c|c|c|c|c|c|c|c|c|}
\hline \multirow{2}{*}{ Solver } & \multirow{2}{*}{ Noise } & \multirow{2}{*}{ Model } & \multicolumn{7}{|c|}{ Approxi mate Parameter Vector } \\
\hline & & & $i=1$ & $i=2$ & $i=\mathbf{3}$ & $i=4$ & $i=5$ & $i=6$ & $i=7$ \\
\hline \multirow{12}{*}{ DE } & $\mathbf{0 . 0 0 0}^{2}$ & Best & -1.000 & 0.800 & 1.000 & 2.800 & -4.800 & 5.700 & 0.600 \\
\hline & & Mean & -1.001 & 0.847 & 1.007 & 2.529 & -4.434 & 5.364 & 0.432 \\
\hline & & Worst & -1.016 & 1.511 & 2.276 & -2.633 & 1.745 & 2.101 & -3.618 \\
\hline & $\mathbf{0 . 0 0 1}^{2}$ & Best & -1.000 & 0.800 & 1.000 & 2.800 & -4.800 & 5.700 & 0.600 \\
\hline & & Mean & -0.996 & 0.858 & 0.857 & 2.561 & -4.422 & 5.176 & 0.518 \\
\hline & & Worst & -0.994 & 0.802 & -3.561 & -0.824 & 1.318 & -1.543 & -2.164 \\
\hline & $\mathbf{0 . 0 1 0}^{2}$ & Best & -1.000 & 0.800 & 1.000 & 2.800 & -4.800 & 5.700 & 0.600 \\
\hline & & Mean & -1.001 & 0.855 & 0.883 & 2.366 & -4.185 & 5.110 & 0.307 \\
\hline & & Worst & -0.994 & 0.802 & -3.561 & -0.824 & 1.318 & -1.543 & -2.164 \\
\hline & $0.100^{2}$ & Best & -1.000 & 0.800 & 1.000 & 2.802 & -4.800 & 5.699 & 0.600 \\
\hline & & Mean & -1.000 & 0.819 & 0.956 & 2.749 & -4.695 & 5.507 & 0.601 \\
\hline & & Worst & -1.000 & 0.800 & 1.000 & 2.802 & -4.800 & 5.699 & 0.00 \\
\hline \multirow{12}{*}{ GAs } & $\mathbf{0 . 0 0 0}^{2}$ & Best & -1.000 & 0.800 & 1.000 & 2.800 & -4.800 & 5.700 & 0.600 \\
\hline & & Mean & -1.000 & 0.800 & 0.999 & 2.802 & -4.803 & 5.703 & 0.600 \\
\hline & & Worst & -1.000 & 0.800 & 1.000 & 2.800 & -4.800 & 5.700 & 0.600 \\
\hline & $\mathrm{O.001}^{2}$ & Best & -1.000 & 0.800 & 0.999 & 2.803 & -4.805 & 5.707 & 0.599 \\
\hline & & Mean & -1.000 & 0.800 & 0.999 & 2.802 & -4.803 & 5.703 & 0.600 \\
\hline & & Worst & -1.000 & 0.800 & 1.000 & 2.800 & -4.800 & 5.700 & 0.600 \\
\hline & $0.010^{2}$ & Best & -1.000 & 0.800 & 1.000 & 2.800 & -4.799 & 5.700 & 0.600 \\
\hline & & Mean & -1.000 & 0.800 & 0.999 & 2.802 & -4.804 & 5.705 & 0.600 \\
\hline & & Worst & -1.000 & 0.800 & 1.000 & 2.800 & -4.800 & 5.700 & 0.600 \\
\hline & $\mathbf{0 . 1 0 0}^{2}$ & Best & -1.000 & 0.800 & 1.000 & 2.799 & -4.799 & 5.700 & 0.600 \\
\hline & & Mean & -1.000 & 0.800 & 0.999 & 2.802 & -4.803 & 5.705 & 0.600 \\
\hline & & Worst & -1.000 & 0.800 & 1.000 & 2.799 & -4.799 & 5.700 & 0.600 \\
\hline \multicolumn{3}{|c|}{ True Values } & -1.000 & 0.800 & 1.000 & 2.800 & -4.800 & 5.700 & 0.600 \\
\hline
\end{tabular}




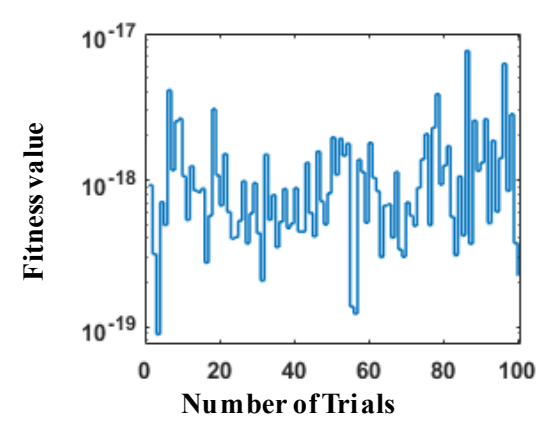

(a) ESM-1 with $\sigma^{2}=0$

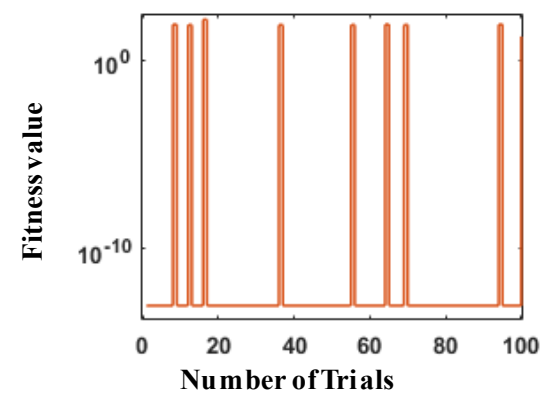

(d) ESM-II with $\sigma^{2}=0.001^{2}$

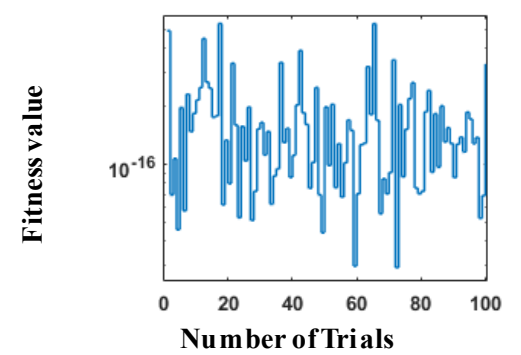

(g) ESM-III with $\sigma^{2}=0$

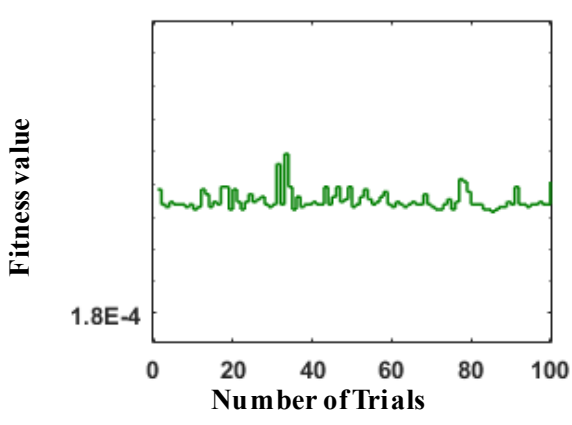

(b) ESM-1 with $\sigma^{2}=0.1^{2}$

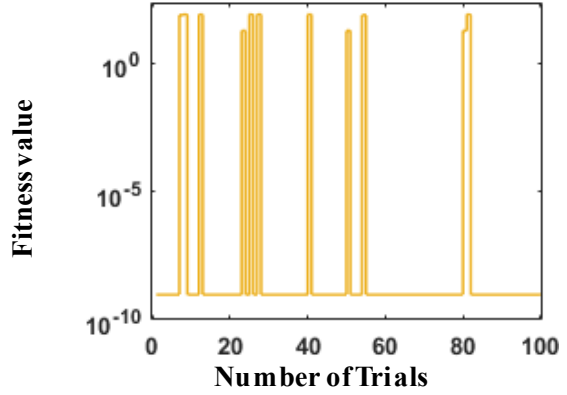

(e) ESM-II with $\sigma^{2}=0.01^{2}$

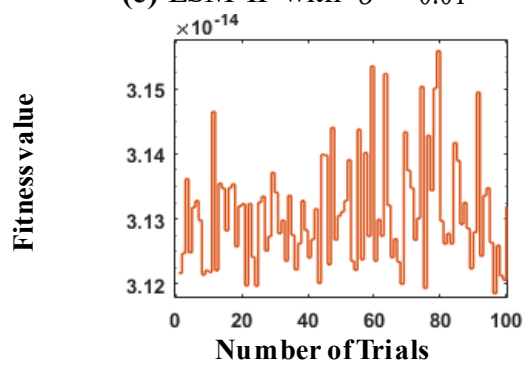

(h) ESM-III with $\sigma^{2}=0.001^{2}$

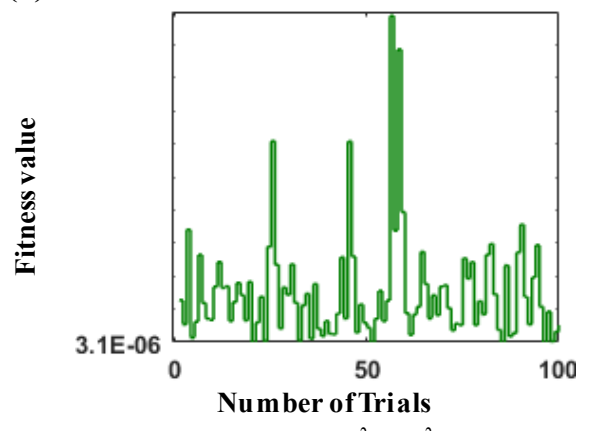

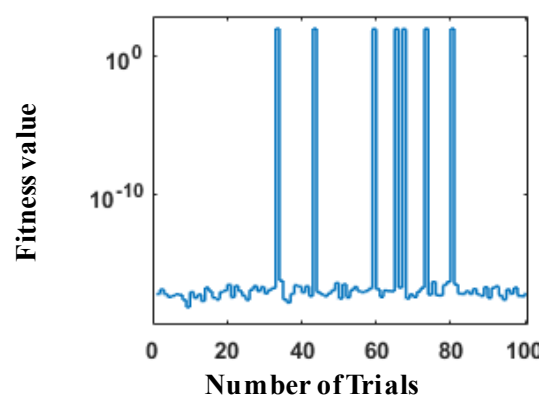

(c) ESM-II with $\sigma^{2}=0$

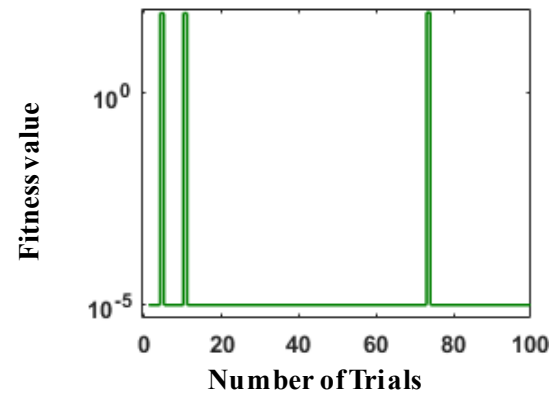

(f) ESM-II with $\sigma^{2}=0.1^{2}$

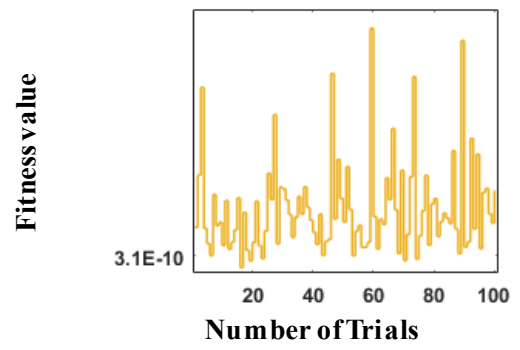

(i) ESM-III with $\sigma^{2}=0.01^{2}$

(j) ESM-III $\sigma^{2}=0.1^{2}$

Fig. 8 Fitness plots for variants of ESM systems using GAs

Accuracy and convergence of both DE and GAs are analyzed through global evaluation metrics given in equations (30) - (33), with $I_{e}=100$ and results are presented in Table 6 for all three ESM systems for all four noise variances. Generally, the magnitude of accuracy for global metrics based on mean fitness lie close to $10^{-04}$ to $10^{-18}$ in case of ESM-I, $10^{-05}$ to $10^{-17}$ for ESM-II, and $10^{-06}$ to $10^{-16}$ in case of ESM-III, for both DE and GAs, while similar trends for performance indices based on $G M A E_{\vartheta}, G N S E_{\vartheta}$, and $G T I C_{\vartheta}$ is observed for all the designed schemes. Very small magnitudes 
of the global operators are generally acquired for all variants which verify the worth of the designed schemes for providing accurate, consistent, and convergent solutions for parameter estimation problem of ESM systems.

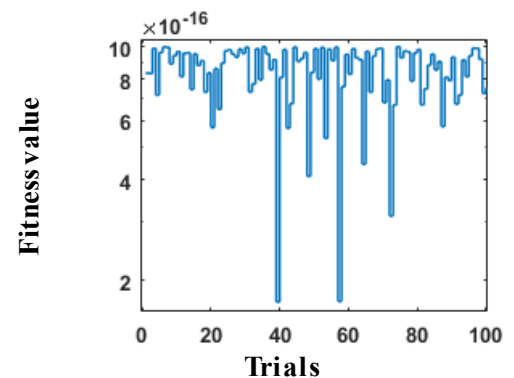

(a) ESM-I with $\sigma^{2}=0$

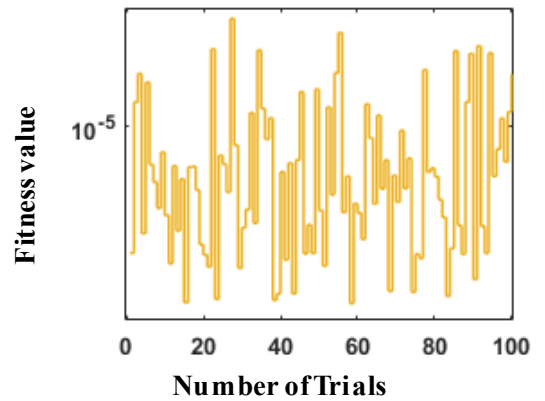

(d) ESM-II with $\sigma^{2}=0.001^{2}$

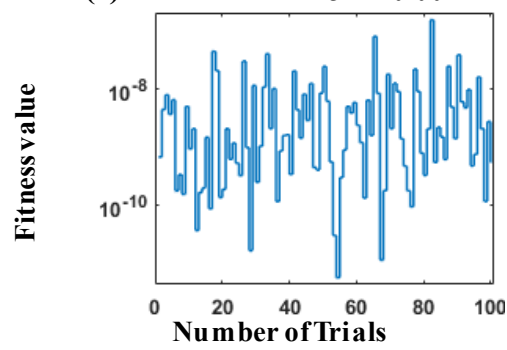

(g) ESM-III with $\sigma^{2}=0$

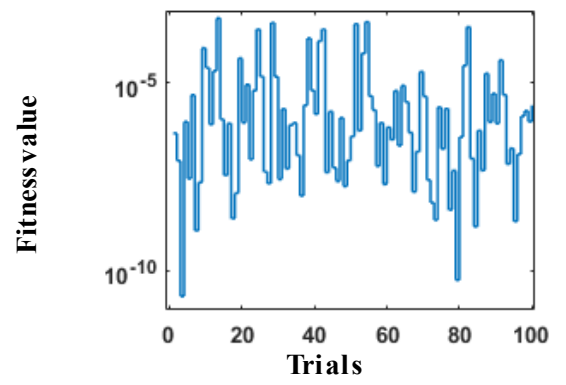

(b) ESM-I with $\sigma^{2}=0.1^{2}$

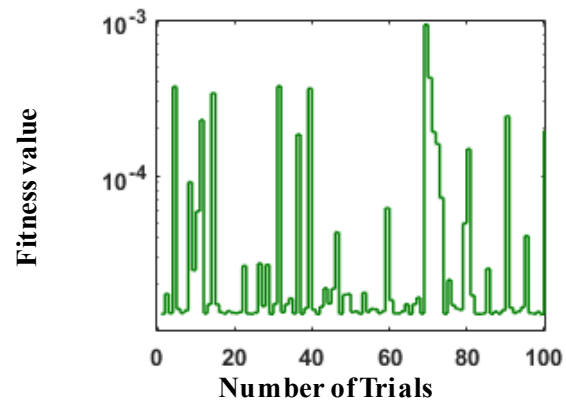

(e) ESM-II with $\sigma^{2}=0.01^{2}$

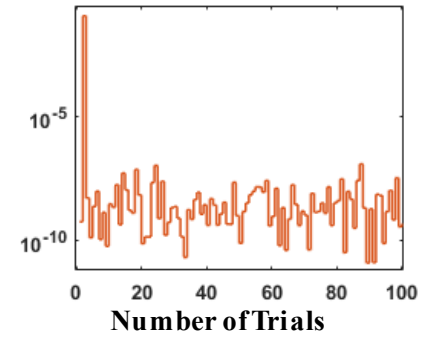

(h) ESM-III with $\sigma^{2}=0.001^{2}$

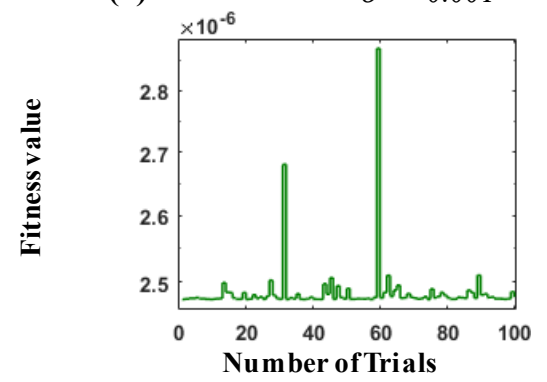

(j) ESM-III with $\sigma^{2}=0.1^{2}$

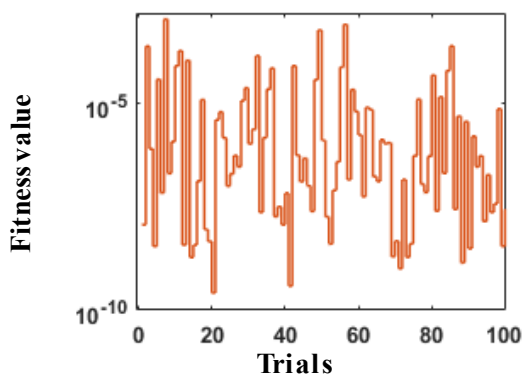

(c) ESM-II with $\sigma^{2}=0$

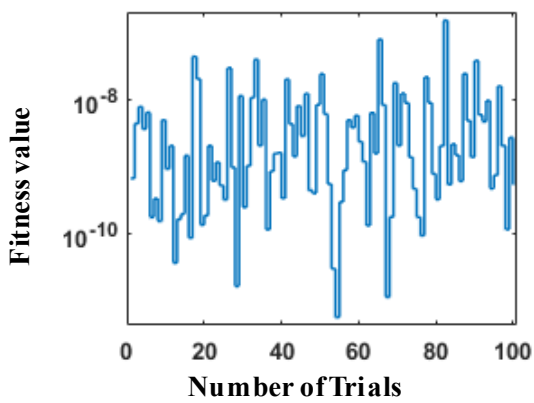

(f) ESM-II with $\sigma^{2}=0.1^{2}$

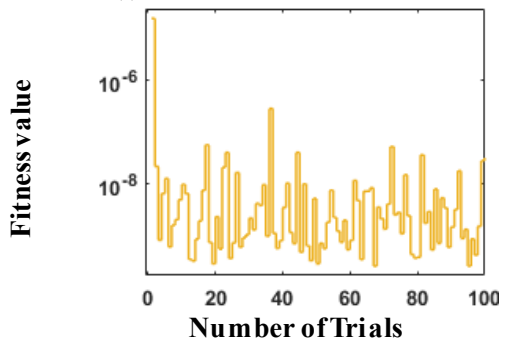

(i) ESM-III with $\sigma^{2}=0.01^{2}$

Fig. 9 Fitness plot for variants of ESM systems using DE 


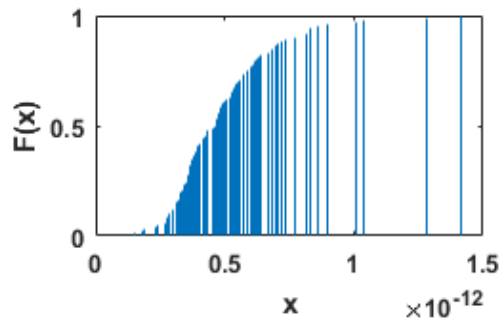

(a) Results of DE for ESM-I with $\sigma^{2}=0.00^{2}$

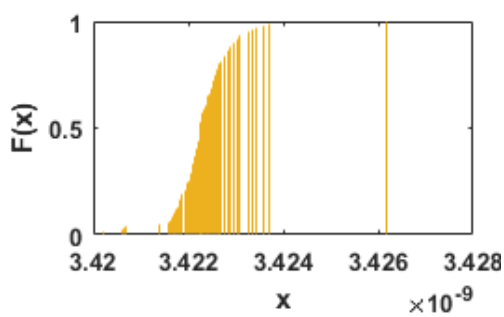

(c) Results of GAs for ESM-I with, $\sigma^{2}=0.01^{2}$

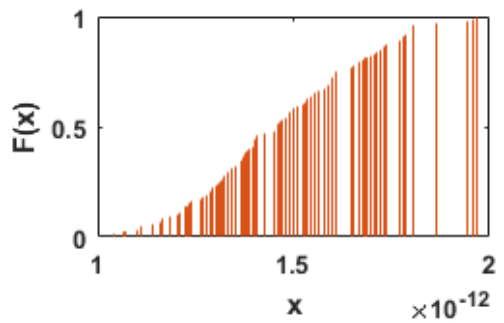

(b) Results of DE for ESM-I with $\sigma^{2}=0.001^{2}$

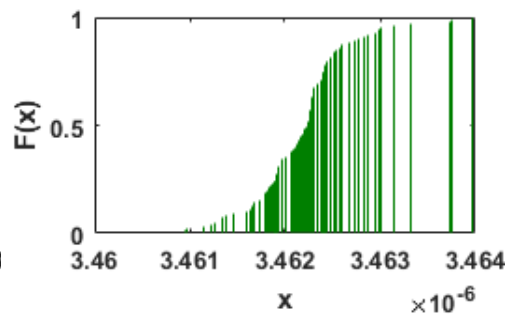

(d) Results of GAs for ESM-I with, $\sigma^{2}=0.1^{2}$

Fig. 10 Plots of TIC indices for variants of ESM-1 system

Table 5: Results of statistical operators for variants of ESM-III system

\begin{tabular}{|c|c|c|c|c|c|c|c|c|c|c|c|}
\hline \multirow{2}{*}{ Solver } & \multirow{2}{*}{ Noise } & \multirow{2}{*}{ Model } & \multicolumn{9}{|c|}{ Approxi mate Parameter Vector } \\
\hline & & & $i=1$ & $i=2$ & $i=\mathbf{3}$ & $i=4$ & $i=5$ & $i=6$ & $i=7$ & $i=8$ & $i=9$ \\
\hline \multirow{12}{*}{ DE } & $0.000^{2}$ & Best & -1.094 & 0.109 & 1.000 & -0.249 & $-2.78 \mathrm{E}-02$ & $1.90 \mathrm{E}-03$ & $-7.83 \mathrm{E}-06$ & $1.95 \mathrm{E}-08$ & $2.36 \mathrm{E}-08$ \\
\hline & & Mean & -1.094 & 0.109 & 1.000 & -0.249 & $-2.78 \mathrm{E}-02$ & $1.90 \mathrm{E}-03$ & $-7.83 \mathrm{E}-06$ & 1.81E-08 & $2.36 \mathrm{E}-08$ \\
\hline & & Worst & -1.094 & 0.109 & 1.000 & -0.249 & $-2.78 \mathrm{E}-02$ & $1.90 \mathrm{E}-03$ & $-7.83 \mathrm{E}-06$ & 2.83E-08 & $2.36 \mathrm{E}-08$ \\
\hline & $0.001^{2}$ & Best & -1.094 & 0.109 & 1.000 & -0.249 & $-2.78 \mathrm{E}-02$ & $1.90 \mathrm{E}-03$ & $-7.77 \mathrm{E}-06$ & $2.29 \mathrm{E}-08$ & $2.36 \mathrm{E}-08$ \\
\hline & & Mean & -1.094 & 0.109 & 1.000 & -0.249 & $-2.78 \mathrm{E}-02$ & $1.90 \mathrm{E}-03$ & $-7.76 \mathrm{E}-06$ & $2.91 \mathrm{E}-08$ & $2.36 \mathrm{E}-08$ \\
\hline & & Worst & -1.094 & 0.109 & 1.000 & -0.249 & $-2.78 \mathrm{E}-02$ & $1.90 \mathrm{E}-03$ & $-7.76 \mathrm{E}-06$ & 3.49E-08 & $2.36 \mathrm{E}-08$ \\
\hline & $0.010^{2}$ & Best & -1.094 & 0.109 & 1.000 & -0.249 & $-2.78 \mathrm{E}-02$ & $1.90 \mathrm{E}-03$ & $-1.24 \mathrm{E}-06$ & $1.18 \mathrm{E}-06$ & $2.36 \mathrm{E}-08$ \\
\hline & & Mean & -1.094 & 0.109 & 1.000 & -0.249 & $-2.78 \mathrm{E}-02$ & $1.90 \mathrm{E}-03$ & $-1.24 \mathrm{E}-06$ & $1.19 \mathrm{E}-06$ & $2.36 \mathrm{E}-08$ \\
\hline & & Worst & -1.094 & 0.109 & 1.000 & -0.249 & $-2.78 \mathrm{E}-02$ & $1.90 \mathrm{E}-03$ & $-1.25 \mathrm{E}-06$ & $1.19 \mathrm{E}-06$ & $2.36 \mathrm{E}-08$ \\
\hline & $0.100^{2}$ & Best & -1.095 & 0.109 & 1.000 & -0.249 & $-2.78 \mathrm{E}-02$ & $1.81 \mathrm{E}-03$ & $6.51 \mathrm{E}-04$ & $1.19 \mathrm{E}-04$ & $2.33 \mathrm{E}-08$ \\
\hline & & Mean & -1.095 & 0.109 & 1.000 & -0.249 & $-2.78 \mathrm{E}-02$ & $1.81 \mathrm{E}-03$ & $6.51 \mathrm{E}-04$ & $1.19 \mathrm{E}-04$ & $2.33 \mathrm{E}-08$ \\
\hline & & Worst & -1.095 & 0.109 & 1.000 & -0.249 & $-2.78 \mathrm{E}-02$ & $1.81 \mathrm{E}-03$ & $6.51 \mathrm{E}-04$ & $1.19 \mathrm{E}-04$ & $2.33 \mathrm{E}-08$ \\
\hline \multirow{12}{*}{ GAs } & 0.000 & Best & -1.094 & 0.109 & 1.000 & -0.249 & $-2.79 \mathrm{E}-02$ & $1.93 \mathrm{E}-03$ & $-1.29 \mathrm{E}-05$ & $-1.22 \mathrm{E}-05$ & $2.36 \mathrm{E}-08$ \\
\hline & & Mean & -1.094 & 0.109 & 1.000 & -0.249 & $-2.78 \mathrm{E}-02$ & $1.89 \mathrm{E}-03$ & $-3.43 \mathrm{E}-06$ & $4.60 \mathrm{E}-06$ & $2.36 \mathrm{E}-08$ \\
\hline & & Worst & -1.094 & 0.109 & 1.000 & -0.249 & $-2.73 \mathrm{E}-02$ & $1.88 \mathrm{E}-03$ & $2.43 \mathrm{E}-05$ & 7.49E-06 & $2.34 \mathrm{E}-08$ \\
\hline & $0.001^{2}$ & Best & -1.094 & 0.109 & 1.000 & -0.249 & $-2.77 \mathrm{E}-02$ & $1.90 \mathrm{E}-03$ & $-6.73 \mathrm{E}-07$ & $1.19 \mathrm{E}-06$ & $2.36 \mathrm{E}-08$ \\
\hline & & Mean & -1.094 & 0.109 & 0.990 & -0.247 & $-2.78 \mathrm{E}-02$ & $1.89 \mathrm{E}-03$ & $-6.48 \mathrm{E}-06$ & $1.86 \mathrm{E}-06$ & $2.97 \mathrm{E}-05$ \\
\hline & & Worst & -1.094 & 0.109 & 1.000 & -0.249 & $-2.78 \mathrm{E}-02$ & $1.90 \mathrm{E}-03$ & $1.03 \mathrm{E}-06$ & $2.70 \mathrm{E}-06$ & $2.36 \mathrm{E}-08$ \\
\hline & $0.010^{2}$ & Best & -1.094 & 0.109 & 1.000 & -0.249 & $-2.76 \mathrm{E}-02$ & $2.00 \mathrm{E}-03$ & $2.52 \mathrm{E}-06$ & $-2.85 \mathrm{E}-05$ & $2.35 \mathrm{E}-08$ \\
\hline & & Mean & -1.094 & 0.109 & 1.000 & -0.249 & $-2.79 \mathrm{E}-02$ & $1.90 \mathrm{E}-03$ & $-8.70 \mathrm{E}-06$ & $1.64 \mathrm{E}-06$ & $2.36 \mathrm{E}-08$ \\
\hline & & Worst & -1.094 & 0.109 & 1.000 & -0.249 & $-2.78 \mathrm{E}-02$ & $1.93 \mathrm{E}-03$ & $-7.98 \mathrm{E}-06$ & $-1.06 \mathrm{E}-05$ & $2.36 \mathrm{E}-08$ \\
\hline & $0.100^{2}$ & Best & -1.094 & 0.109 & 1.000 & -0.249 & $-2.78 \mathrm{E}-02$ & $2.05 \mathrm{E}-03$ & $1.90 \mathrm{E}-04$ & $2.71 \mathrm{E}-05$ & $2.33 \mathrm{E}-08$ \\
\hline & & Mean & -1.094 & 0.109 & 1.000 & -0.249 & $-2.78 \mathrm{E}-02$ & 2.04E-03 & $1.89 \mathrm{E}-04$ & $3.21 \mathrm{E}-05$ & $2.33 \mathrm{E}-08$ \\
\hline & & Worst & -1.000 & 0.800 & 1.000 & 2.799 & -4.799 & 5.700 & 0.600 & $2.11 \mathrm{E}-05$ & $2.78 \mathrm{E}-08$ \\
\hline \multicolumn{3}{|c|}{ rue Values } & -1.000 & 0.800 & 1.000 & 2.800 & -4.800 & 5.700 & 0.600 & $1.95 \mathrm{E}-08$ & $2.36 \mathrm{E}-08$ \\
\hline
\end{tabular}




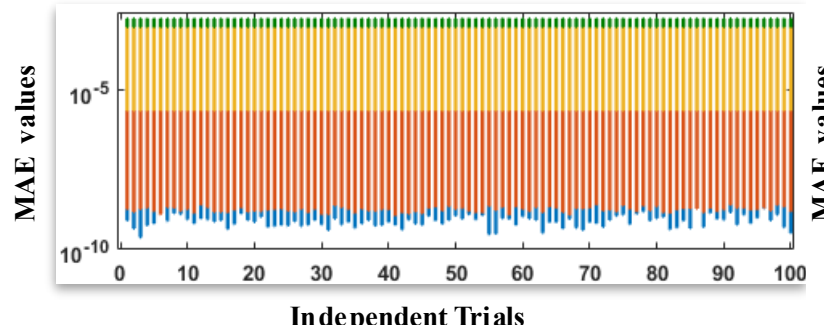

(a). Results of DE for ESM-I

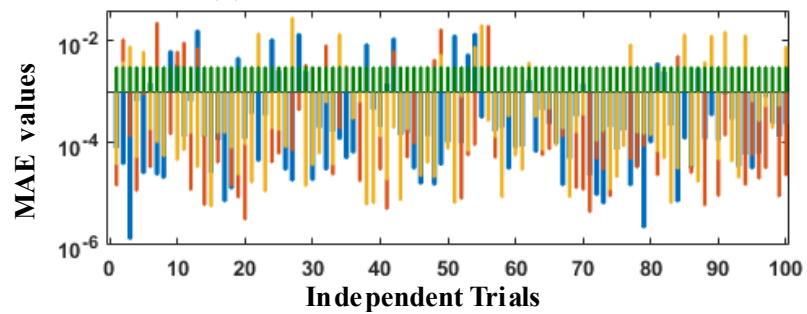

(c). Results of GAs for ESM-I

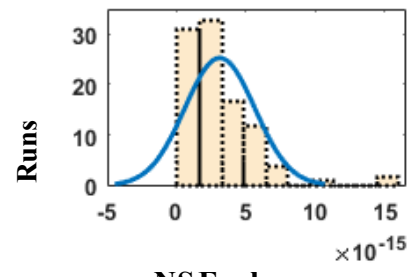

NS Evalues

(e). DE for $\sigma^{2}=0$

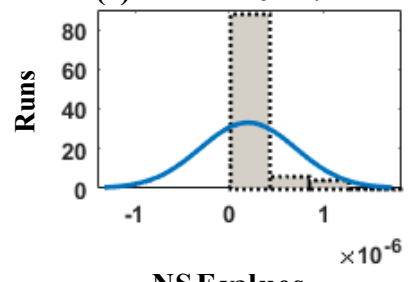

NSEvalues

(i). GAs for $\sigma^{2}=0$

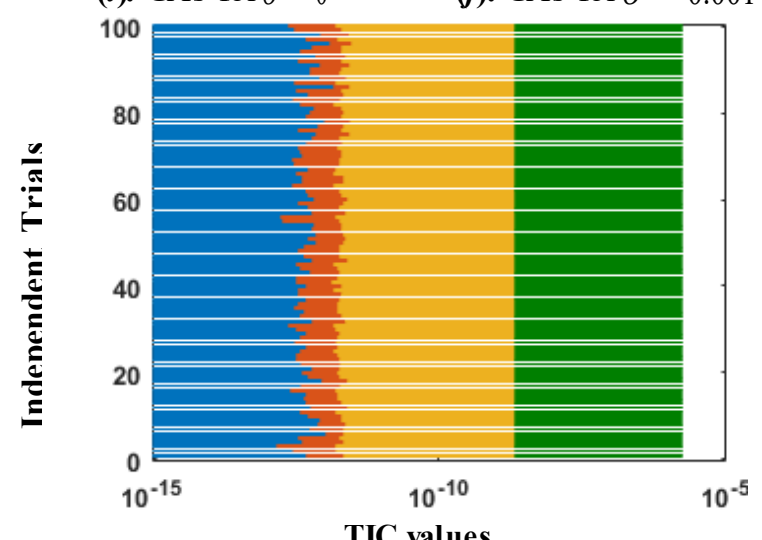

(m). Results for DE for EMS-I

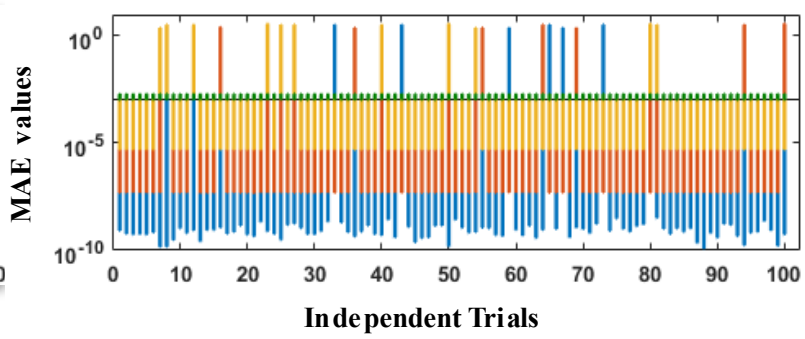

(b). Results of DE for ESM-1I

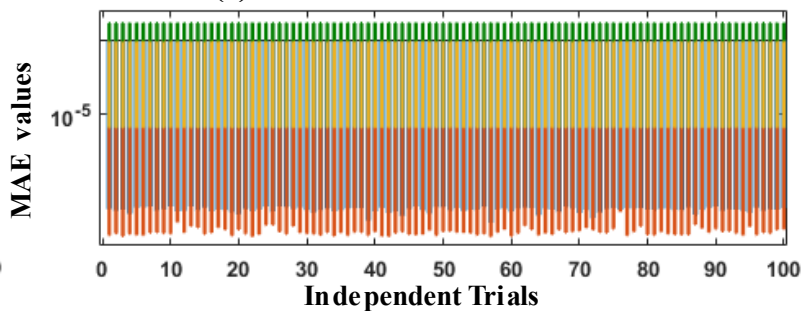

(d). Results of GAs for ESM-II
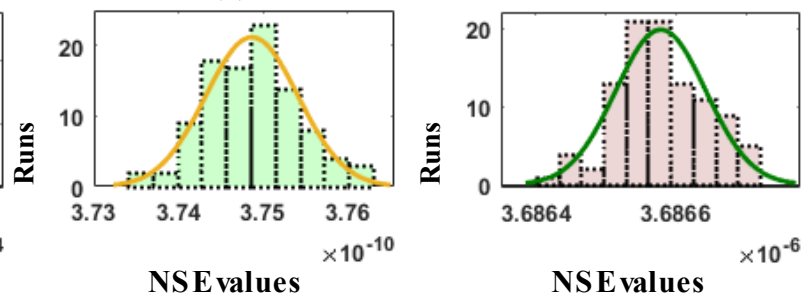

(g). DE for $\sigma^{2}=0.01^{2}$

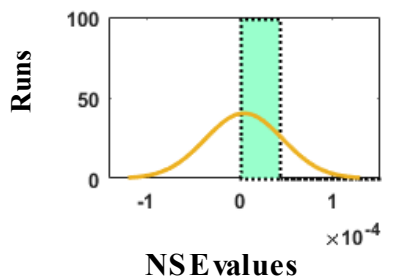

(k). GAs for $\sigma^{2}=0.01^{2}$

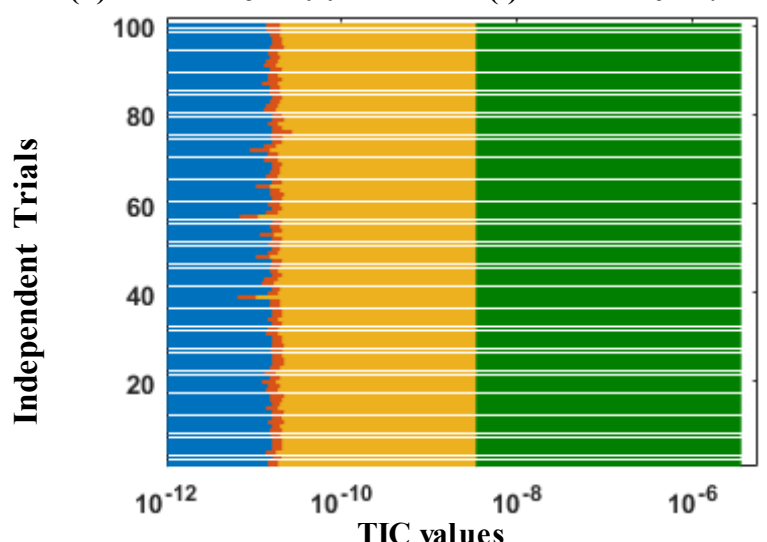

(n). Results for GAs for EMS-I

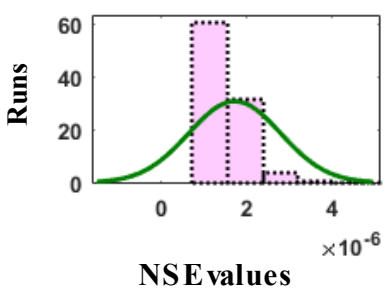

(l). GAs for $\sigma^{2}=0.1^{2}$

$-\sigma^{2}=0^{2}-\sigma^{2}=0.001^{2}-\sigma^{2}=0.01^{2}-\sigma^{2}=0.1^{2}$

Fig. 11. Results based on MAE, NSE and TIC indices through bar, histogram and stack-bar graphics for ESM-I and II. 


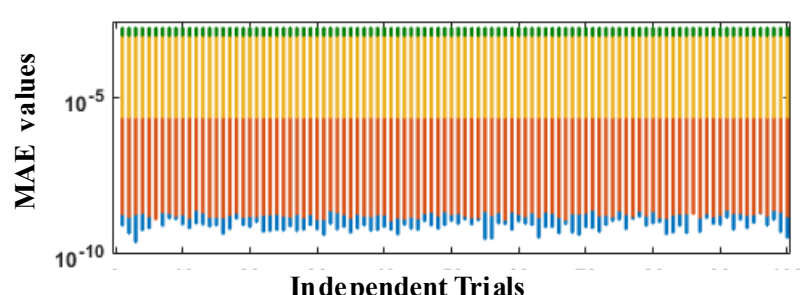

(a). Results of DE for ESM-III
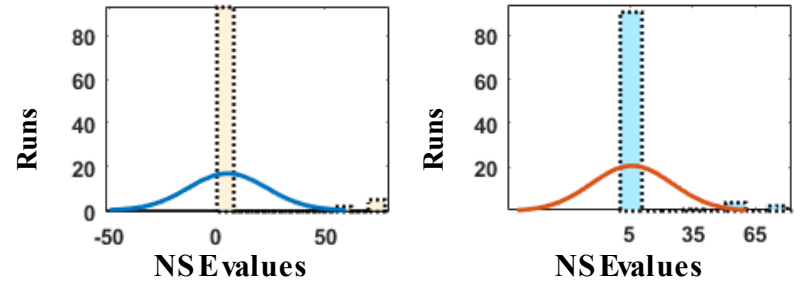

(e). DE for $\sigma^{2}=0$

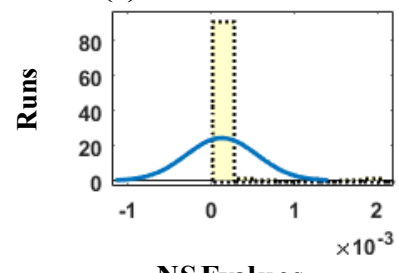

NS Evalues (f). DE for $\sigma^{2}=0.001^{2}$

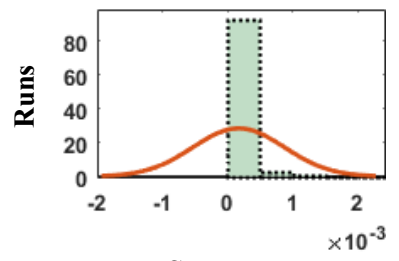

NSE values

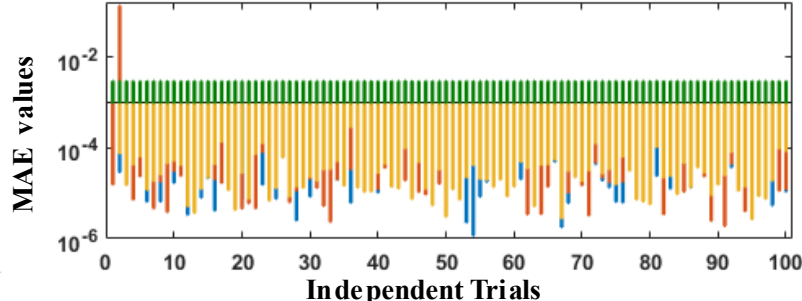

(b). Results of GAs for ESM-III

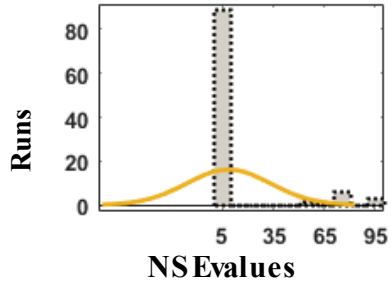

(g). DE for $\sigma^{2}=0.01^{2}$

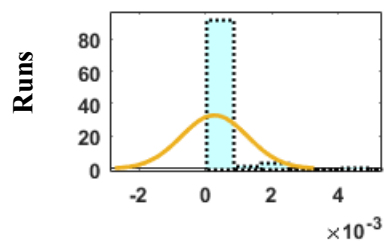

NS Evalues

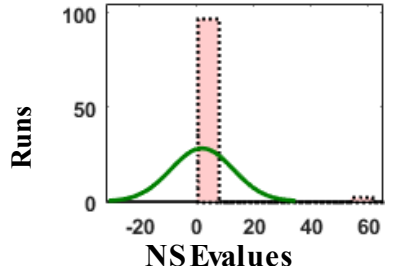

(h). DE for $\sigma^{2}=0.1^{2}$

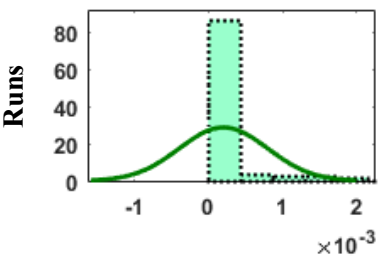

NS Evalues

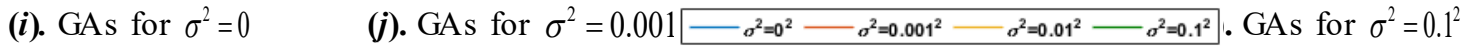

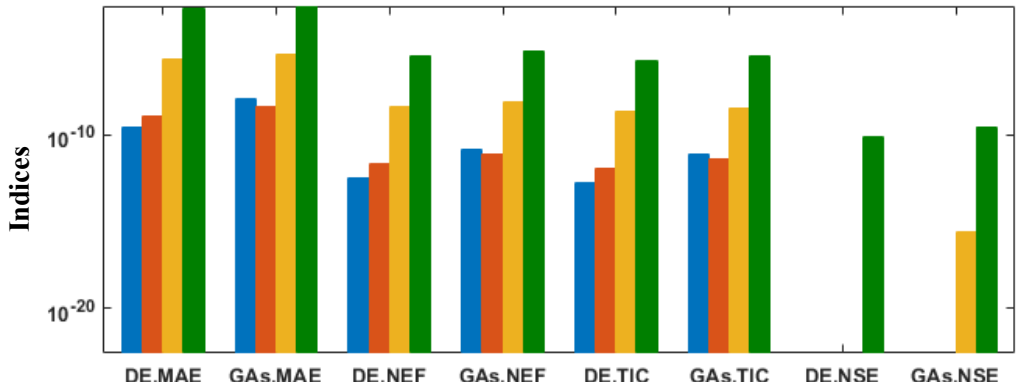

(m). Analysis through performance indices

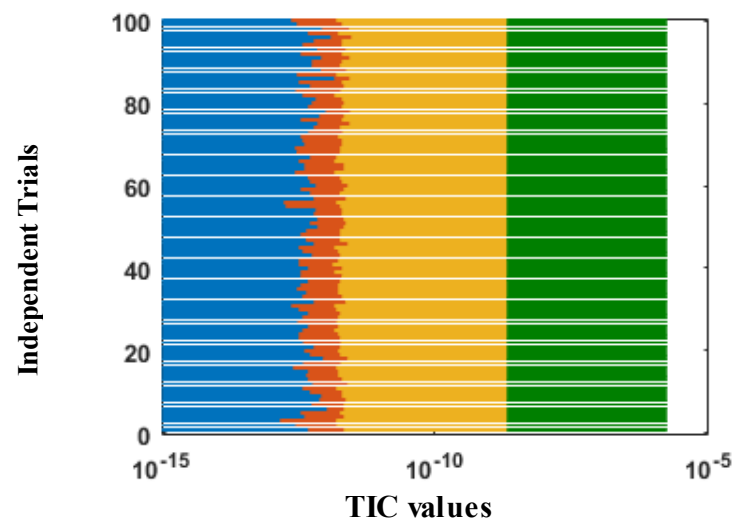

(n). Results of DE for ESM-III

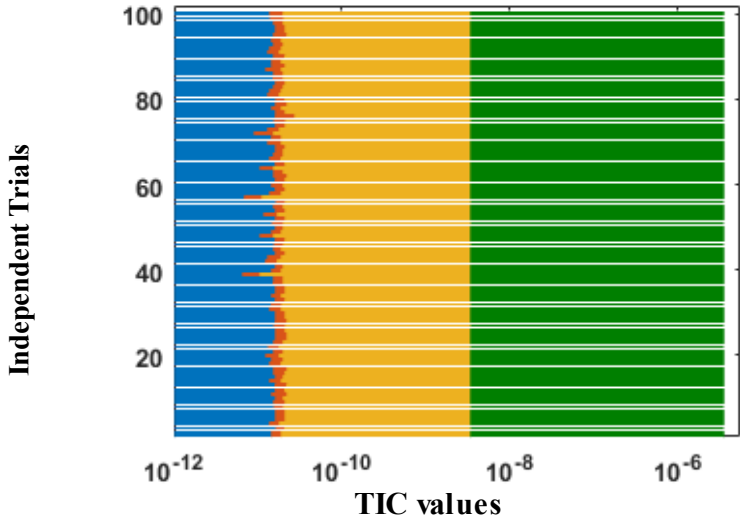

(i). Results of GAs for ESM-III

$-\sigma^{2}=0^{2}-\sigma^{2}=0.001^{2}-\sigma^{2}=0.01^{2}-\sigma^{2}=0.1^{2}$

Fig. 12 Results based on MAE, NSE and TIC indices through bar, histogram, and stack-bar graphics for ESM-III. 
Table 6: Statistical analysis on global operators for each case of all ESM models

\begin{tabular}{|c|c|c|c|c|c|c|c|c|c|c|}
\hline \multirow{3}{*}{ Solver } & \multirow{3}{*}{ ESM } & \multirow{3}{*}{$\begin{array}{c}\text { Noise } \\
\sigma^{2}\end{array}$} & \multicolumn{8}{|c|}{ Global Operators } \\
\hline & & & \multicolumn{2}{|c|}{ Fitness $\varepsilon$} & \multicolumn{2}{|c|}{ MWD $\delta$} & \multicolumn{3}{|c|}{ NSE } & \multirow{2}{*}{$\begin{array}{r}\text { TIC } \\
\text { STD }\end{array}$} \\
\hline & & & Mean & STD & Mean & STD & Mean & STD & Mean & \\
\hline \multirow{12}{*}{ DE } & \multirow{4}{*}{ I } & $\overline{0^{2}}$ & $1.13 \mathrm{E}-18$ & $1.14 \mathrm{E}-18$ & $8.19 \mathrm{E}-10$ & $3.50 \mathrm{E}-10$ & $0.00 \mathrm{E}+00$ & $0.00 \mathrm{E}+00$ & $4.99 \mathrm{E}-13$ & $2.17 \mathrm{E}-13$ \\
\hline & & $10^{-4}$ & $3.34 \mathrm{E}-12$ & $8.64 \mathrm{E}-19$ & $1.81 \mathrm{E}-09$ & $3.28 \mathrm{E}-10$ & $0.00 \mathrm{E}+00$ & $0.00 \mathrm{E}+00$ & $1.47 \mathrm{E}-12$ & $2.17 \mathrm{E}-13$ \\
\hline & & $10^{-2}$ & 3.34E-08 & $1.39 \mathrm{E}-18$ & $2.40 \mathrm{E}-06$ & 4.57E-10 & $0.00 \mathrm{E}+00$ & $0.00 \mathrm{E}+00$ & $2.05 \mathrm{E}-09$ & $2.51 \mathrm{E}-13$ \\
\hline & & $10^{-1}$ & $1.83 \mathrm{E}-04$ & $1.17 \mathrm{E}-18$ & $1.87 \mathrm{E}-03$ & $3.52 \mathrm{E}-10$ & 7.33E-11 & $9.79 \mathrm{E}-17$ & $1.75 \mathrm{E}-06$ & 7.92E-14 \\
\hline & \multirow{4}{*}{ II } & $\mathbf{0}^{2}$ & $5.62 \mathrm{E}+00$ & $2.06 \mathrm{E}+01$ & $2.00 \mathrm{E}-01$ & 7.40E-01 & 4.93E+00 & $1.82 \mathrm{E}+01$ & 4.89E-02 & $1.79 \mathrm{E}-01$ \\
\hline & & $10^{-4}$ & $7.22 \mathrm{E}+00$ & $2.49 \mathrm{E}+01$ & $2.40 \mathrm{E}-01$ & 7.82E-01 & $5.88 \mathrm{E}+00$ & $1.94 \mathrm{E}+01$ & $6.18 \mathrm{E}-02$ & $1.99 \mathrm{E}-01$ \\
\hline & & $10^{-2}$ & $7.00 \mathrm{E}+00$ & $2.20 \mathrm{E}+01$ & $3.35 \mathrm{E}-01$ & $9.69 \mathrm{E}-01$ & $8.54 \mathrm{E}+00$ & $2.48 \mathrm{E}+01$ & $8.25 \mathrm{E}-02$ & $2.38 \mathrm{E}-01$ \\
\hline & & $10^{-1}$ & $2.37 \mathrm{E}+00$ & $1.36 \mathrm{E}+01$ & $1.87 \mathrm{E}-03$ & $3.52 \mathrm{E}-10$ & $1.89 \mathrm{E}+00$ & $1.09 \mathrm{E}+01$ & $2.00 \mathrm{E}-02$ & $1.14 \mathrm{E}-01$ \\
\hline & \multirow{4}{*}{ III } & $\overline{0^{2}}$ & $1.59 \mathrm{E}-16$ & $1.04 \mathrm{E}-16$ & $6.68 \mathrm{E}-09$ & 2.44E-09 & $3.12 \mathrm{E}-15$ & $2.52 \mathrm{E}-15$ & 8.97E-09 & $3.41 \mathrm{E}-09$ \\
\hline & & $10^{-4}$ & $3.13 \mathrm{E}-14$ & 8.34E-17 & $2.46 \mathrm{E}-08$ & 2.13E-09 & 4.07E-14 & $5.09 \mathrm{E}-15$ & $3.45 \mathrm{E}-08$ & $2.18 \mathrm{E}-09$ \\
\hline & & $10^{-2}$ & $3.12 \mathrm{E}-10$ & $1.15 \mathrm{E}-16$ & $2.13 \mathrm{E}-06$ & 2.76E-09 & $3.75 \mathrm{E}-10$ & $5.46 \mathrm{E}-13$ & $3.32 \mathrm{E}-06$ & 2.42E-09 \\
\hline & & $10^{-1}$ & $3.12 \mathrm{E}-06$ & $1.30 \mathrm{E}-16$ & $1.87 \mathrm{E}-03$ & $3.52 \mathrm{E}-10$ & $3.69 \mathrm{E}-06$ & $6.44 \mathrm{E}-11$ & $3.29 \mathrm{E}-04$ & $2.88 \mathrm{E}-09$ \\
\hline \multirow{12}{*}{ GAs } & \multirow{4}{*}{ I } & 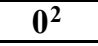 & $8.42 \mathrm{E}-16$ & $1.72 \mathrm{E}-16$ & 2.35E-08 & 3.63E-09 & $0.00 \mathrm{E}+00$ & $0.00 \mathrm{E}+00$ & $1.48 \mathrm{E}-11$ & $1.78 \mathrm{E}-12$ \\
\hline & & $10^{-4}$ & 7.57E-12 & 4.04E-17 & $6.00 \mathrm{E}-09$ & $1.97 \mathrm{E}-09$ & $0.00 \mathrm{E}+00$ & $0.00 \mathrm{E}+00$ & $5.02 \mathrm{E}-12$ & $9.17 \mathrm{E}-13$ \\
\hline & & $10^{-2}$ & 7.56E-08 & $2.62 \mathrm{E}-17$ & $4.19 \mathrm{E}-06$ & $1.41 \mathrm{E}-09$ & $2.22 \mathrm{E}-16$ & $0.00 \mathrm{E}+00$ & $3.42 \mathrm{E}-09$ & $6.92 \mathrm{E}-13$ \\
\hline & & $10^{-1}$ & 4.01E-04 & $1.56 \mathrm{E}-10$ & 3.04E-03 & 3.09E-06 & $2.87 \mathrm{E}-10$ & 8.75E-14 & $3.46 \mathrm{E}-06$ & $5.28 \mathrm{E}-10$ \\
\hline & \multirow{4}{*}{ II } & $\overline{0^{2}}$ & $3.20 \mathrm{E}-05$ & $9.38 \mathrm{E}-05$ & $1.38 \mathrm{E}-03$ & 3.23E-03 & $1.25 \mathrm{E}-04$ & 4.26E-04 & $3.20 \mathrm{E}-04$ & 7.44E-04 \\
\hline & & $10^{-4}$ & 4.02E-05 & 1.47E-04 & $1.52 \mathrm{E}-03$ & $3.82 \mathrm{E}-03$ & $1.71 \mathrm{E}-04$ & 7.02E-04 & $3.50 \mathrm{E}-04$ & $8.78 \mathrm{E}-04$ \\
\hline & & $10^{-2}$ & 6.02E-05 & 2.17E-04 & $2.03 \mathrm{E}-03$ & $4.76 \mathrm{E}-03$ & 2.72E-04 & $9.96 \mathrm{E}-04$ & $4.68 \mathrm{E}-04$ & $1.10 \mathrm{E}-03$ \\
\hline & & $10^{-1}$ & $5.75 \mathrm{E}-05$ & $1.25 \mathrm{E}-04$ & $3.04 \mathrm{E}-03$ & 3.09E-06 & $2.04 \mathrm{E}-04$ & $5.96 \mathrm{E}-04$ & $4.80 \mathrm{E}-04$ & $9.14 \mathrm{E}-04$ \\
\hline & \multirow{4}{*}{ III } & $\overline{0^{2}}$ & 7.68E-09 & $1.88 \mathrm{E}-08$ & $3.20 \mathrm{E}-05$ & 3.23E-05 & $1.97 \mathrm{E}-07$ & 5.07E-07 & $5.24 \mathrm{E}-05$ & $5.56 \mathrm{E}-05$ \\
\hline & & $10^{-4}$ & $1.18 \mathrm{E}-03$ & $1.18 \mathrm{E}-02$ & $1.42 \mathrm{E}-03$ & $1.39 \mathrm{E}-02$ & 3.97E-02 & 3.97E-01 & 4.01E-03 & $3.95 \mathrm{E}-02$ \\
\hline & & $10^{-2}$ & $1.57 \mathrm{E}-07$ & $1.48 \mathrm{E}-06$ & $5.21 \mathrm{E}-05$ & $1.95 \mathrm{E}-04$ & 4.38E-06 & $4.16 \mathrm{E}-05$ & 8.77E-05 & $3.50 \mathrm{E}-04$ \\
\hline & & $10^{-1}$ & 2.49E-06 & 4.45E-08 & $3.04 \mathrm{E}-03$ & 3.09E-06 & $1.72 \mathrm{E}-06$ & $1.08 \mathrm{E}-06$ & $2.20 \mathrm{E}-04$ & 4.68E-05 \\
\hline
\end{tabular}

Complexity of the designed schemes is determined in terms of time consumed for execution by each solver, cycles completed, function evaluated. Results based on these measures are obtained for 100 trials of each solver and are provided in Table 7 for mean and standard deviation measures. The values of mean execution time, generations and functions evaluations are close to $7.389 \pm$ $0.45,207.55 \pm 1.45$ and $37563.740 \pm 7.45$ for ESM-I, 9.839 $\pm 0.640,182.6 \pm 11.161$ and $38527.6 \pm$ 9.8 for ESM-II and $18.50 \pm 0.8,233.750 \pm 6.28$ and $63345.250 \pm 18.438$ for ESM-III in case of DE while the for GAs the respective magnitudes are around $11.161 \pm 0.57,300$ and 60200 for

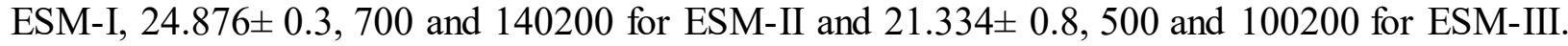
It is seen that DE outperforms GAs in terms of each complexity operator based on execution time, generations consumed and functions evaluated. All numerical experimentations for ESM models are conducted on Dell notebook 35212, Intel Core(TM) i3, CPU @ $1.90 \mathrm{GHz}$ processor and 6.00 GB of RAM. 
Table 7: Results of complexity measures for all three ESM models

\begin{tabular}{|c|c|c|c|c|c|c|c|c|}
\hline \multirow{3}{*}{ Solver } & \multirow{3}{*}{ ESM } & \multirow{3}{*}{$\begin{array}{c}\text { Noise } \\
\sigma^{2}\end{array}$} & \multicolumn{6}{|c|}{ Complexity Operators } \\
\hline & & & \multicolumn{2}{|c|}{ Time } & \multicolumn{2}{|c|}{ Generations } & \multicolumn{2}{|c|}{ Function Counts } \\
\hline & & & Mean & STD & Mean & STD & Mean & STD \\
\hline \multirow{12}{*}{ DE } & \multirow{4}{*}{ I } & $\mathbf{0}^{2}$ & 7.362 & 0.595 & 208.540 & 14.082 & 37744.740 & 7.362 \\
\hline & & $10^{-4}$ & 7.389 & 0.496 & 207.540 & 13.693 & 37563.740 & 7.389 \\
\hline & & $10^{-2}$ & 7.524 & 0.531 & 209.260 & 14.385 & 37875.060 & 7.524 \\
\hline & & $10^{-1}$ & 7.489 & 0.471 & 211.820 & 12.481 & 38338.420 & 7.489 \\
\hline & \multirow{4}{*}{ II } & $\mathbf{0}^{2}$ & 10.572 & 0.754 & 183.040 & 6.399 & 38620.440 & 10.572 \\
\hline & & $10^{-4}$ & 9.839 & 0.640 & 182.600 & 11.161 & 38527.600 & 9.839 \\
\hline & & $10^{-2}$ & 9.920 & 0.398 & 183.710 & 7.458 & 38761.810 & 9.920 \\
\hline & & $10^{-1}$ & 10.058 & 0.697 & 182.170 & 6.495 & 38436.870 & 10.058 \\
\hline & \multirow{4}{*}{ III } & $\mathbf{0}^{2}$ & 21.638 & 17.654 & 234.720 & 6.529 & 63011.950 & 21.638 \\
\hline & & $10^{-4}$ & 18.043 & 0.514 & 233.750 & 6.288 & 63345.250 & 18.043 \\
\hline & & $10^{-2}$ & 17.899 & 0.762 & 233.790 & 5.661 & 63356.090 & 17.899 \\
\hline & & $10^{-1}$ & 20.209 & 18.238 & 234.430 & 6.557 & 63529.530 & 20.209 \\
\hline \multirow{12}{*}{ GAs } & \multirow{4}{*}{ I } & $0^{2}$ & 10.369 & 0.000 & 214.010 & 26.558 & 60200.000 & 10.369 \\
\hline & & $10^{-4}$ & 11.161 & 0.599 & 300.000 & 0.000 & 60200.000 & 11.161 \\
\hline & & $10^{-2}$ & 9.723 & 0.282 & 300.000 & 0.000 & 60200.000 & 9.723 \\
\hline & & $10^{-1}$ & 10.642 & 0.556 & 300.000 & 0.000 & 60200.000 & 10.642 \\
\hline & \multirow{4}{*}{$1 \mathrm{I}$} & $\mathbf{0}^{2}$ & 24.679 & 0.971 & 700.000 & 0.000 & 140200.000 & 24.679 \\
\hline & & $10^{-4}$ & 25.239 & 0.272 & 700.000 & 0.000 & 140200.000 & 25.239 \\
\hline & & $10^{-2}$ & 24.876 & 0.284 & 700.000 & 0.000 & 140200.000 & 24.876 \\
\hline & & $10^{-1}$ & 23.956 & 0.207 & 700.000 & 0.000 & 140200.000 & 23.956 \\
\hline & \multirow{4}{*}{ III } & $\mathbf{0}^{2}$ & 20.638 & 0.269 & 500.000 & 0.000 & 100200.000 & 20.638 \\
\hline & & $10^{-4}$ & 21.334 & 0.134 & 500.000 & 0.000 & 100200.000 & 21.334 \\
\hline & & $10^{-2}$ & 19.987 & 0.154 & 500.000 & 0.000 & 100200.000 & 19.987 \\
\hline & & $10^{-1}$ & 21.012 & 0.184 & 500.000 & 0.000 & 100200.000 & 21.012 \\
\hline
\end{tabular}

In order to compare the performance of the proposed $\mathrm{DE}$ with other state-of-the-art heuristics the results of DE are also compared with particle swarm optimization (PSO), simulated annealing (SA) and pattern search (PS) techniques. The results of PSO, SA and PS in terms of accuracy and complexity measures are listed in Table 8 . It can be observed that the fitness values lie in the range of $10^{-04}$ to $10^{-10}, 10^{-03}$ to $10^{-04}$, and $10^{-06}$ to $10^{-14}$ for ESM-I, ESM-II, and ESM-III, respectively, in case of PSO, while for PS respective values lie close to $10^{-04}$ to $10^{-9}, 10^{+01}$ to $10^{-04}$, and $10^{-01}$ to $10^{-}$ ${ }^{08}$, whereas in case of SA respective magnitudes ranges between $10^{-01}$ to $10^{-02}, 10^{+01}$ to $10^{-01}$, and $10^{+03}$ to $10^{-01}$. Generally, good results in terms of accuracy are obtained by PSO while performance degraded considerably for PS and SA algorithms. However, PSO consumed more time and function counts than other two algorithms, hence PSO is considered more complex. Comparison of the results of PSO with DE and GAs, show that PSO achieve the similar accuracy as that of GAs but considerably 'degraded performance of accuracy than that of DE. Moreover, the complexity of DE algorithm for optimization of decision variable is much better than that of GAs and PSO algorithms. 
Table 8: Comparison of the performance on basis of best runs of all three algorithms for each variant of ESM models

\begin{tabular}{|c|c|c|c|c|c|c|c|c|c|}
\hline \multirow{2}{*}{ Solver } & \multirow{2}{*}{ ESM } & \multirow{2}{*}{$\sigma^{2}$} & \multicolumn{5}{|c|}{ Accuracy } & \multicolumn{2}{|c|}{ Complexity } \\
\hline & & & $\varepsilon$ & $\delta$ & MAE & NSE & TIC & Time & FC \\
\hline \multirow{12}{*}{ PSO } & \multirow{4}{*}{ I } & 0 & $4.08 \mathrm{E}-09$ & $6.55 \mathrm{E}-08$ & $5.43 \mathrm{E}-05$ & $2.58 \mathrm{E}-14$ & $3.27 \mathrm{E}-08$ & 250.8 & 1600200 \\
\hline & & $10^{-3}$ & $6.58 \mathrm{E}-10$ & $6.55 \mathrm{E}-08$ & $2.02 \mathrm{E}-05$ & $4.00 \mathrm{E}-15$ & $1.29 \mathrm{E}-08$ & 212.1 & 1600200 \\
\hline & & $10^{-2}$ & 2.04E-07 & 7.87E-08 & $3.65 \mathrm{E}-05$ & $1.47 \mathrm{E}-14$ & $2.48 \mathrm{E}-08$ & 212.5 & 1600200 \\
\hline & & $10^{-1}$ & $8.12 \mathrm{E}-04$ & $7.87 \mathrm{E}-08$ & $2.43 \mathrm{E}-03$ & $1.77 \mathrm{E}-10$ & $2.72 \mathrm{E}-06$ & 214.1 & 1600200 \\
\hline & \multirow{4}{*}{ II } & $\mathbf{0}$ & $1.73 \mathrm{E}-04$ & $6.55 \mathrm{E}-08$ & 8.93E-03 & $8.59 \mathrm{E}-04$ & $2.12 \mathrm{E}-03$ & 571.5 & 4066493 \\
\hline & & $10^{-3}$ & $2.45 \mathrm{E}-04$ & $6.29 \mathrm{E}-07$ & $1.06 \mathrm{E}-02$ & $1.21 \mathrm{E}-03$ & $2.52 \mathrm{E}-03$ & 631.1 & 4000040 \\
\hline & & $10^{-2}$ & $5.76 \mathrm{E}-04$ & $6.28 \mathrm{E}-07$ & $8.63 \mathrm{E}-03$ & $7.96 \mathrm{E}-04$ & $2.04 \mathrm{E}-03$ & 639.4 & 4000040 \\
\hline & & $10^{-1}$ & $1.30 \mathrm{E}-03$ & 4.05E-07 & $2.43 \mathrm{E}-03$ & $3.45 \mathrm{E}-04$ & $1.34 \mathrm{E}-03$ & 630.8 & 4000040 \\
\hline & \multirow{4}{*}{ III } & $\mathbf{0}$ & $0.00 \mathrm{E}+00$ & $6.55 \mathrm{E}-08$ & $0.00 \mathrm{E}+00$ & $0.00 \mathrm{E}+00$ & $0.00 \mathrm{E}+00$ & 1926.5 & 10000100 \\
\hline & & $10^{-3}$ & $1.90 \mathrm{E}-14$ & $6.28 \mathrm{E}-07$ & $4.12 \mathrm{E}-08$ & $1.27 \mathrm{E}-13$ & $6.12 \mathrm{E}-08$ & 1885.3 & 10000100 \\
\hline & & $10^{-2}$ & $1.90 \mathrm{E}-10$ & 4.05E-07 & $4.12 \mathrm{E}-06$ & $1.27 \mathrm{E}-09$ & $6.12 \mathrm{E}-06$ & 1900.9 & 10000100 \\
\hline & & $10^{-1}$ & $1.90 \mathrm{E}-06$ & $6.28 \mathrm{E}-07$ & $2.43 \mathrm{E}-03$ & $1.27 \mathrm{E}-05$ & $6.12 \mathrm{E}-04$ & 1932.2 & 10000100 \\
\hline \multirow{12}{*}{ PS } & \multirow{4}{*}{ I } & $\mathbf{0}$ & $1.05 \mathrm{E}-09$ & $3.33 \mathrm{E}-08$ & $1.34 \mathrm{E}-05$ & $6.66 \mathrm{E}-15$ & $1.66 \mathrm{E}-08$ & 7.20 & 2444 \\
\hline & & $10^{-3}$ & $1.36 \mathrm{E}-11$ & 4.60E-06 & $1.60 \mathrm{E}-06$ & $0.00 \mathrm{E}+00$ & $1.30 \mathrm{E}-09$ & 8.19 & 2396 \\
\hline & & $10^{-2}$ & 7.31E-08 & $1.39 \mathrm{E}-04$ & $6.78 \mathrm{E}-06$ & 4.44E-16 & 4.78E-09 & 8.19 & 2393 \\
\hline & & $10^{-1}$ & $3.02 \mathrm{E}-04$ & 7.12E-05 & $2.62 \mathrm{E}-03$ & $2.09 \mathrm{E}-10$ & $2.95 \mathrm{E}-06$ & 8.28 & 3643 \\
\hline & \multirow{4}{*}{ II } & $\mathbf{0}$ & $3.69 \mathrm{E}-04$ & $1.12 \mathrm{E}-06$ & $7.46 \mathrm{E}-03$ & $5.53 \mathrm{E}-04$ & $1.70 \mathrm{E}-03$ & 11.63 & 4165 \\
\hline & & $10^{-4}$ & $2.81 \mathrm{E}-03$ & $2.24 \mathrm{E}-06$ & $2.40 \mathrm{E}-02$ & $5.68 \mathrm{E}-03$ & $5.47 \mathrm{E}-03$ & 11.60 & 4263 \\
\hline & & $10^{-2}$ & $1.05 \mathrm{E}-03$ & $1.38 \mathrm{E}-05$ & $1.37 \mathrm{E}-02$ & $1.84 \mathrm{E}-03$ & $3.10 \mathrm{E}-03$ & 11.56 & 4489 \\
\hline & & $10^{-1}$ & $1.88 \mathrm{E}+01$ & 7.12E-05 & $2.62 \mathrm{E}-03$ & $9.47 \mathrm{E}+01$ & $8.80 \mathrm{E}-01$ & 11.43 & 5032 \\
\hline & \multirow{4}{*}{ III } & $\mathbf{0}$ & 8.33E-08 & $4.45 \mathrm{E}-06$ & $1.30 \mathrm{E}-04$ & $1.97 \mathrm{E}-06$ & $2.41 \mathrm{E}-04$ & 11.90 & 5502 \\
\hline & & $10^{-4}$ & $8.22 \mathrm{E}-08$ & 7.33E-06 & $1.40 \mathrm{E}-04$ & $2.07 \mathrm{E}-06$ & $2.47 \mathrm{E}-04$ & 12.73 & 5712 \\
\hline & & $10^{-2}$ & $1.53 \mathrm{E}-07$ & 3.31E-06 & $1.84 \mathrm{E}-04$ & 3.82E-06 & $3.35 \mathrm{E}-04$ & 12.93 & 6286 \\
\hline & & $10^{-1}$ & $2.46 \mathrm{E}-01$ & 7.12E-05 & $2.62 \mathrm{E}-03$ & $6.91 \mathrm{E}+00$ & 4.94E-01 & 13.14 & 6365 \\
\hline \multirow{12}{*}{ SA } & \multirow{4}{*}{ I } & $\mathbf{0}$ & $1.63 \mathrm{E}-02$ & $1.31 \mathrm{E}-04$ & $8.62 \mathrm{E}-02$ & $1.02 \mathrm{E}-07$ & $6.54 \mathrm{E}-05$ & 2.99 & 18001 \\
\hline & & $10^{-3}$ & $1.67 \mathrm{E}-01$ & $1.31 \mathrm{E}-04$ & $3.26 \mathrm{E}-01$ & $1.05 \mathrm{E}-06$ & $2.09 \mathrm{E}-04$ & 2.78 & 16860 \\
\hline & & $10^{-2}$ & 6.03E-02 & $6.59 \mathrm{E}-04$ & $2.21 \mathrm{E}-01$ & $3.79 \mathrm{E}-07$ & $1.26 \mathrm{E}-04$ & 4.17 & 16650 \\
\hline & & $10^{-1}$ & $4.95 \mathrm{E}-02$ & $6.59 \mathrm{E}-04$ & $1.62 \mathrm{E}-01$ & $8.28 \mathrm{E}-07$ & $1.86 \mathrm{E}-04$ & 3.41 & 18001 \\
\hline & \multirow{4}{*}{ II } & 0 & $1.26 \mathrm{E}+01$ & $1.31 \mathrm{E}-04$ & $1.79 \mathrm{E}+00$ & $3.00 \mathrm{E}+01$ & 4.92E-01 & 3.56 & 19110 \\
\hline & & $10^{-3}$ & $2.93 \mathrm{E}+01$ & 4.60E-04 & $1.89 \mathrm{E}+00$ & $3.41 \mathrm{E}+01$ & $5.08 \mathrm{E}-01$ & 3.87 & 19576 \\
\hline & & $10^{-2}$ & $1.31 \mathrm{E}+01$ & $2.59 \mathrm{E}-04$ & $1.95 \mathrm{E}+00$ & $3.53 \mathrm{E}+01$ & $5.19 \mathrm{E}-01$ & 4.01 & 20878 \\
\hline & & $10^{-1}$ & $2.28 \mathrm{E}+00$ & $1.55 \mathrm{E}-04$ & $1.62 \mathrm{E}-01$ & $3.15 \mathrm{E}+01$ & $5.07 \mathrm{E}-01$ & 4.80 & 21000 \\
\hline & \multirow{4}{*}{ III } & $\mathbf{0}$ & $1.77 \mathrm{E}+01$ & $1.31 \mathrm{E}-04$ & $1.17 \mathrm{E}+00$ & $6.74 \mathrm{E}+01$ & $8.10 \mathrm{E}-01$ & 4.92 & 21060 \\
\hline & & $10^{-3}$ & $1.03 \mathrm{E}+03$ & $2.59 \mathrm{E}-04$ & $3.00 \mathrm{E}+00$ & $4.68 \mathrm{E}+02$ & $8.48 \mathrm{E}-01$ & 5.10 & 22534 \\
\hline & & $10^{-2}$ & $6.66 \mathrm{E}+01$ & $1.55 \mathrm{E}-04$ & $1.32 \mathrm{E}+00$ & $2.30 \mathrm{E}+02$ & $8.61 \mathrm{E}-01$ & 5.56 & 24378 \\
\hline & & $10^{-1}$ & $1.09 \mathrm{E}+01$ & $2.59 \mathrm{E}-04$ & $1.62 \mathrm{E}-01$ & $7.48 \mathrm{E}+01$ & 7.33E-01 & 5.94 & 24989 \\
\hline
\end{tabular}

The analysis of accuracy level of PSO, PS and SA is further conducted on the basis of 100 independent executions of each algorithm for optimization of decision variable of EMS models. Results of statistics in terms of best, mean and worst indices are given in Table 9 for ESM-I along with the desired parameters, while, the results for ESM-II and ESM-III are provided in Tables 10 and 11 respectively, for each scenarios. It is observed that with the increase in noise level $\sigma^{2}=$ $0.001^{2}$ to $0.1^{2}$ degradation in the performance is noticed in case of both PSO and PS, however, 
both the algorithms are significantly applicable for finding the optimal ESM parameters with reasonable accuracy, while the results of SA are relatively poor. Comparing the performance with proposed DE algorithm, it can be evidently seen that performance of PSO is almost similar to GAs, but relative better that PS and SA algorithms, however inferior than that of DE based method.

Table 9: Comparison through results of statistics for variants of ESM-I system

\begin{tabular}{|c|c|c|c|c|c|c|c|c|}
\hline \multirow{2}{*}{ Solver } & \multirow{2}{*}{$\sigma^{2}$} & \multirow{2}{*}{ Model } & \multicolumn{6}{|c|}{ Approximate Parameter Vector } \\
\hline & & & $i=1$ & $i=2$ & $i=\mathbf{3}$ & $i=4$ & $i=5$ & $i=6$ \\
\hline \multirow{12}{*}{ PSO } & \multirow{3}{*}{$\mathbf{0}^{2}$} & Best & -1.998 & 0.998 & 0.002 & 6.899 & 0.041 & 2389.7 \\
\hline & & Mean & -1.999 & 0.998 & 0.002 & 6.899 & 0.041 & 2389.7 \\
\hline & & Worst & -1.998 & 0.998 & 0.003 & 6.900 & 0.041 & 2389.7 \\
\hline & \multirow{3}{*}{$10^{-4}$} & Best & -1.998 & 0.999 & 0.002 & 6.899 & 0.041 & 2389.7 \\
\hline & & Mean & -1.999 & 0.999 & 0.002 & 6.899 & 0.041 & 2389.7 \\
\hline & & Worst & -2.000 & 0.998 & 0.002 & 6.899 & 0.041 & 2389.7 \\
\hline & \multirow{3}{*}{$10^{-2}$} & Best & -1.999 & 0.998 & 0.002 & 6.899 & 0.041 & 2389.7 \\
\hline & & Mean & -1.999 & 0.998 & 0.002 & 6.899 & 0.041 & 2389.7 \\
\hline & & Worst & -2.000 & 0.998 & 0.002 & 6.899 & 0.041 & 2389.7 \\
\hline & \multirow{3}{*}{$10^{-1}$} & Best & -2.000 & 0.986 & 0.002 & 6.899 & 0.041 & 2389.7 \\
\hline & & Mean & -2.000 & 0.986 & 0.002 & 6.899 & 0.041 & 2389.7 \\
\hline & & Worst & -2.000 & 0.986 & 0.002 & 6.899 & 0.041 & 2389.7 \\
\hline \multirow{12}{*}{$\mathbf{S A}$} & \multirow{3}{*}{$\mathbf{0}^{2}$} & Best & -1.956 & 0.936 & $\begin{array}{c}-0.080 \\
\end{array}$ & 6.869 & 0.094 & 2389.7 \\
\hline & & Mean & -1.718 & 1.003 & 0.061 & 6.681 & 0.029 & 2389.7 \\
\hline & & Worst & -1.332 & 0.091 & 0.730 & 5.867 & -0.632 & 2389.3 \\
\hline & \multirow{3}{*}{$10^{-4}$} & Best & -1.940 & 1.139 & 0.012 & 6.801 & -0.050 & 2389.7 \\
\hline & & Mean & -1.717 & 1.027 & 0.023 & 6.736 & 0.041 & 2389.6 \\
\hline & & Worst & -0.929 & 0.685 & 0.762 & 6.391 & -0.625 & 2389.5 \\
\hline & \multirow{3}{*}{$10^{-2}$} & Best & -1.813 & 1.003 & 0.022 & 6.929 & 0.070 & 2389.6 \\
\hline & & Mean & -1.682 & 0.995 & -0.029 & 6.674 & 0.082 & 2389.7 \\
\hline & & Worst & -1.033 & 1.633 & 0.528 & 6.494 & 0.677 & 2389.6 \\
\hline & \multirow{3}{*}{$10^{-1}$} & Best & -1.919 & 1.091 & -0.026 & 6.883 & -0.021 & 2389.8 \\
\hline & & Mean & -1.670 & 0.990 & 0.015 & 6.665 & 0.101 & 2389.7 \\
\hline & & Worst & -1.640 & 1.585 & 0.140 & 6.103 & 1.434 & 2388.8 \\
\hline \multirow{12}{*}{ PS } & \multirow{3}{*}{$0^{2}$} & Best & $\begin{array}{c}-1.998 \\
\end{array}$ & 0.999 & 0.002 & 6.899 & 0.041 & 2389.7 \\
\hline & & Mean & -1.968 & 1.002 & 0.019 & 6.900 & 0.048 & 2389.7 \\
\hline & & Worst & -1.999 & 0.993 & 0.642 & 6.898 & 0.039 & 2389.7 \\
\hline & \multirow{3}{*}{$10^{-4}$} & Best & -1.999 & 0.999 & 0.002 & 6.899 & 0.041 & 2389.7 \\
\hline & & Mean & -1.941 & 1.004 & 0.015 & 6.900 & 0.068 & 2389.7 \\
\hline & & Worst & -0.438 & 0.994 & -0.002 & 6.886 & 0.036 & 2389.7 \\
\hline & \multirow{3}{*}{$10^{-2}$} & Best & -1.999 & 0.999 & 0.002 & 6.899 & 0.041 & 2389.7 \\
\hline & & Mean & -1.952 & 1.005 & 0.030 & 6.900 & 0.060 & 2389.7 \\
\hline & & Worst & -1.999 & 0.996 & -0.002 & 6.897 & 1.253 & 2389.6 \\
\hline & \multirow{3}{*}{$10^{-1}$} & Best & -2.000 & 0.984 & 0.002 & 6.899 & 0.041 & 2389.7 \\
\hline & & Mean & -1.918 & 0.988 & 0.021 & 6.898 & 0.063 & 2389.7 \\
\hline & & Worst & -0.788 & 0.995 & -0.017 & 6.873 & 0.030 & 2389.7 \\
\hline \multicolumn{3}{|c|}{ True Parameter Vector } & -1.998 & 0.998 & 0.002 & 6.899 & 0.041 & 2389.7 \\
\hline
\end{tabular}


Table 10: Comparison through results of statistics for variants of ESM-II system

\begin{tabular}{|c|c|c|c|c|c|c|c|c|c|}
\hline \multirow{2}{*}{ Solver } & \multirow{2}{*}{ Noise } & \multirow{2}{*}{ Model } & \multicolumn{7}{|c|}{ Approximate Parameter Vector } \\
\hline & & & $i=1$ & $i=\mathbf{2}$ & $i=\mathbf{3}$ & $i=4$ & $i=5$ & $i=6$ & $i=7$ \\
\hline \multirow{12}{*}{ PSO } & \multirow{3}{*}{$\mathbf{0 . 0 0 0}^{2}$} & Best & -1.000 & 0.800 & 1.004 & 2.790 & -4.779 & 5.675 & 0.603 \\
\hline & & Mean & -1.000 & 0.800 & 1.044 & 2.701 & -4.600 & 5.456 & 0.627 \\
\hline & & Worst & -1.000 & 0.800 & 1.108 & 2.563 & -4.326 & 5.125 & 0.666 \\
\hline & \multirow{3}{*}{$\mathbf{0 . 0 0 1}^{2}$} & Best & -1.000 & 0.800 & 1.005 & 2.788 & -4.775 & 5.670 & 0.603 \\
\hline & & Mean & -1.000 & 0.800 & 1.081 & 2.639 & -4.457 & 5.284 & 0.650 \\
\hline & & Worst & -1.000 & 0.800 & 1.219 & 2.371 & -3.923 & 4.635 & 0.736 \\
\hline & \multirow{3}{*}{$0.010^{2}$} & Best & -1.000 & 0.800 & 1.004 & 2.790 & -4.782 & 5.674 & 0.602 \\
\hline & & Mean & -1.000 & 0.800 & 1.027 & 2.740 & -4.675 & 5.546 & 0.617 \\
\hline & & Worst & -1.000 & 0.800 & 1.081 & 2.624 & -4.440 & 5.259 & 0.649 \\
\hline & \multirow{3}{*}{$0.100^{2}$} & Best & -1.000 & 0.800 & 1.012 & 2.777 & -4.743 & 5.630 & 0.607 \\
\hline & & Mean & -1.000 & 0.800 & 1.026 & 2.745 & -4.681 & 5.554 & 0.616 \\
\hline & & Worst & -1.000 & 0.800 & 1.101 & 2.585 & -4.356 & 5.158 & 0.662 \\
\hline \multirow{12}{*}{$\mathbf{S A}$} & \multirow{3}{*}{$\mathbf{0 . 0 0 0}^{2}$} & Best & -1.040 & 0.808 & 4.029 & 1.379 & -1.413 & 1.361 & 0.905 \\
\hline & & Mean & -0.989 & 0.799 & 1.830 & 1.158 & -1.380 & 1.679 & 1.293 \\
\hline & & Worst & -1.067 & 0.836 & 1.515 & -3.593 & -3.352 & 5.871 & 1.299 \\
\hline & \multirow{3}{*}{$0.001^{2}$} & Best & -1.073 & 0.809 & 4.511 & 1.950 & -1.345 & 1.036 & -0.067 \\
\hline & & Mean & -0.986 & 0.796 & 1.409 & 1.002 & -1.326 & 1.657 & 1.001 \\
\hline & & Worst & -0.955 & 0.783 & 3.171 & 2.179 & -1.228 & 1.099 & 2.020 \\
\hline & \multirow{3}{*}{$0.010^{2}$} & Best & -1.019 & 0.799 & 4.487 & 1.571 & -1.200 & 1.025 & 1.229 \\
\hline & & Mean & -0.982 & 0.794 & 1.464 & 1.250 & -1.418 & 1.687 & 0.917 \\
\hline & & Worst & -0.918 & 0.779 & -3.361 & -1.170 & 0.849 & -1.036 & -4.169 \\
\hline & \multirow{3}{*}{$0.100^{2}$} & Best & -0.985 & 0.795 & 1.734 & 2.008 & -2.573 & 2.994 & 1.137 \\
\hline & & Mean & -0.979 & 0.792 & 1.586 & 1.242 & -1.146 & 1.269 & 1.126 \\
\hline & & Worst & -0.959 & 0.788 & 2.605 & 0.589 & -1.482 & 1.997 & 2.822 \\
\hline \multirow{12}{*}{ PS } & \multirow{3}{*}{$\mathbf{0 . 0 0 0}^{2}$} & Best & -0.999 & 0.799 & 1.004 & 2.794 & -4.781 & 5.682 & 0.604 \\
\hline & & Mean & -1.020 & 0.826 & -0.782 & 1.293 & -2.050 & 2.549 & -0.475 \\
\hline & & Worst & -1.007 & 0.809 & -3.577 & -0.771 & 1.338 & -1.536 & -2.109 \\
\hline & \multirow{3}{*}{$\mathbf{0 . 0 0 1}^{2}$} & Best & -0.999 & 0.799 & 1.013 & 2.777 & -4.740 & 5.641 & 0.610 \\
\hline & & Mean & -1.032 & 0.840 & -0.639 & 1.322 & -2.047 & 2.544 & -0.451 \\
\hline & & Worst & -1.548 & 1.459 & 0.443 & -4.944 & -0.606 & -4.997 & 0.491 \\
\hline & \multirow{3}{*}{$0.010^{2}$} & Best & -0.999 & 0.799 & 1.008 & 2.787 & -4.765 & 5.667 & 0.606 \\
\hline & & Mean & -1.009 & 0.811 & -0.587 & 1.421 & -2.477 & 2.943 & -0.289 \\
\hline & & Worst & -1.007 & 0.809 & -3.577 & -0.771 & 1.338 & -1.536 & -2.109 \\
\hline & \multirow{3}{*}{$0.100^{2}$} & Best & -1.007 & 0.809 & -3.577 & -0.771 & 1.338 & -1.536 & -2.108 \\
\hline & & Mean & -1.030 & 0.836 & -1.030 & 1.218 & -1.692 & 2.251 & -0.582 \\
\hline & & Worst & -1.007 & 0.809 & -3.577 & -0.771 & 1.338 & -1.536 & -2.108 \\
\hline & \multicolumn{2}{|c|}{ rue Values } & -1.000 & 0.800 & 1.000 & 2.800 & -4.800 & 5.700 & 0.600 \\
\hline
\end{tabular}


Table 11: Comparison through results of statistics for variants of ESM-III system

\begin{tabular}{|c|c|c|c|c|c|c|c|c|c|c|c|}
\hline \multirow{2}{*}{ Solver } & \multirow{2}{*}{ Noise } & \multirow{2}{*}{ Model } & \multicolumn{9}{|c|}{ Approximate Parameter Vector } \\
\hline & & & $i=1$ & $i=2$ & $i=\mathbf{3}$ & $i=4$ & $i=5$ & $i=6$ & $i=7$ & $i=8$ & $i=9$ \\
\hline \multirow{12}{*}{ DE } & $\widehat{0.000^{2}}$ & Best & -1.094 & 0.109 & 1.000 & -0.249 & -0.028 & 0.002 & $-7.83 \mathrm{E}-06$ & $1.78 \mathrm{E}-08$ & $2.36 \mathrm{E}-08$ \\
\hline & & Mean & -1.094 & 0.109 & 1.000 & -0.249 & -0.028 & 0.002 & $-7.83 \mathrm{E}-06$ & $1.78 \mathrm{E}-08$ & $2.36 \mathrm{E}-08$ \\
\hline & & Worst & -1.094 & 0.109 & 1.000 & -0.249 & -0.028 & 0.002 & $-7.83 \mathrm{E}-06$ & $1.78 \mathrm{E}-08$ & 2.36E-08 \\
\hline & $0.001^{2}$ & Best & -1.094 & 0.109 & 1.000 & -0.249 & -0.028 & 0.002 & $-7.84 \mathrm{E}-06$ & $-6.94 \mathrm{E}-08$ & 2.36E-08 \\
\hline & & Mean & -1.094 & 0.109 & 1.000 & -0.249 & -0.028 & 0.002 & $-7.84 \mathrm{E}-06$ & $-6.94 \mathrm{E}-08$ & 2.36E-08 \\
\hline & & Worst & -1.094 & 0.109 & 1.000 & -0.249 & -0.028 & 0.002 & $-7.84 \mathrm{E}-06$ & $-6.94 \mathrm{E}-08$ & 2.36E-08 \\
\hline & $0.010^{2}$ & Best & -1.094 & 0.109 & 1.000 & -0.249 & -0.028 & 0.002 & $-9.12 \mathrm{E}-06$ & $-8.70 \mathrm{E}-06$ & 2.36E-08 \\
\hline & & Mean & -1.094 & 0.109 & 1.000 & -0.249 & -0.028 & 0.002 & $-9.12 \mathrm{E}-06$ & $-8.70 \mathrm{E}-06$ & 2.36E-08 \\
\hline & & Worst & -1.094 & 0.109 & 1.000 & -0.249 & -0.028 & 0.002 & $-9.12 \mathrm{E}-06$ & $-8.70 \mathrm{E}-06$ & 2.36E-08 \\
\hline & $0.100^{2}$ & Best & -1.095 & 0.108 & 1.000 & -0.249 & -0.028 & 0.003 & $-1.37 \mathrm{E}-04$ & $-8.73 \mathrm{E}-04$ & $2.36 \mathrm{E}-08$ \\
\hline & & Mean & -1.095 & 0.108 & 1.000 & -0.249 & -0.028 & 0.003 & $-1.37 \mathrm{E}-04$ & $-8.73 \mathrm{E}-04$ & $2.36 \mathrm{E}-08$ \\
\hline & & Worst & -1.095 & 0.108 & 1.000 & -0.249 & -0.028 & 0.003 & $-1.37 \mathrm{E}-04$ & $-8.73 \mathrm{E}-04$ & 2.36E-08 \\
\hline \multirow{12}{*}{ GAs } & $\widehat{0.000^{2}}$ & Best & 0.851 & -0.361 & 0.694 & 2.380 & 1.413 & 1.160 & $1.68 \mathrm{E}+00$ & $-8.88 \mathrm{E}-01$ & $-8.65 \mathrm{E}-07$ \\
\hline & & Mean & 0.422 & 0.800 & 0.286 & -0.210 & 0.316 & 0.637 & 4.78E-01 & 7.50E-01 & 2.39E-01 \\
\hline & & Worst & 0.851 & -0.361 & 0.694 & 2.380 & 1.413 & 1.160 & $1.68 \mathrm{E}+00$ & $-8.88 \mathrm{E}-01$ & $-8.65 \mathrm{E}-07$ \\
\hline & $\widehat{0.001^{2}}$ & Best & 5.909 & -1.676 & 5.945 & 2.561 & 0.814 & -4.149 & $4.69 \mathrm{E}+00$ & 8.09E-01 & $-1.58 \mathrm{E}-06$ \\
\hline & & Mean & 0.473 & 0.101 & 0.186 & -0.015 & 0.784 & 0.296 & $5.76 \mathrm{E}-01$ & $1.61 \mathrm{E}+00$ & 5.24E-02 \\
\hline & & Worst & -2.805 & -0.157 & -4.276 & -3.841 & -2.976 & 2.560 & $5.43 \mathrm{E}+00$ & $1.69 \mathrm{E}+00$ & $-4.82 \mathrm{E}-07$ \\
\hline & $\overline{0.010^{2}}$ & Best & 1.649 & 1.025 & 1.419 & -1.454 & 1.329 & 1.251 & $-1.57 \mathrm{E}+00$ & $2.42 \mathrm{E}+00$ & 3.22E-05 \\
\hline & & Mean & 0.256 & 0.530 & 0.008 & 0.123 & 0.686 & 0.268 & $6.12 \mathrm{E}-01$ & $-4.37 \mathrm{E}-01$ & $-1.55 \mathrm{E}-02$ \\
\hline & & Worst & 0.470 & 0.790 & -0.217 & 1.213 & 1.374 & 1.432 & $6.25 \mathrm{E}-01$ & 7.92E-01 & $-1.84 \mathrm{E}-07$ \\
\hline & $\widehat{0.100^{2}}$ & Best & 3.247 & -0.798 & 2.903 & -2.901 & -1.623 & -1.754 & 9.44E-01 & $3.55 \mathrm{E}-01$ & $-1.95 \mathrm{E}-05$ \\
\hline & & Mean & 0.308 & 0.662 & -0.024 & 0.105 & 0.389 & 0.333 & $1.27 \mathrm{E}-01$ & $1.64 \mathrm{E}+00$ & 5.92E-03 \\
\hline & & Worst & 0.691 & 4.288 & 0.546 & -0.553 & 4.499 & 2.280 & $-3.39 \mathrm{E}+00$ & $2.87 \mathrm{E}+00$ & 5.49E-05 \\
\hline \multirow{12}{*}{ DE } & $\overline{0.000^{2}}$ & Best & -1.094 & 0.109 & 1.000 & -0.249 & -0.027 & 0.002 & $1.02 \mathrm{E}-04$ & $-8.83 \mathrm{E}-06$ & $2.33 \mathrm{E}-08$ \\
\hline & & Mean & -1.093 & 0.110 & 0.097 & -0.037 & -0.028 & -0.214 & $-9.10 \mathrm{E}-03$ & $-1.41 \mathrm{E}-01$ & $-8.49 \mathrm{E}-06$ \\
\hline & & Worst & -0.906 & 0.267 & -0.681 & 0.681 & -0.028 & -5.115 & $-1.37 \mathrm{E}-01$ & $-1.61 \mathrm{E}+00$ & $-1.27 \mathrm{E}-04$ \\
\hline & $0.001^{2}$ & Best & -1.094 & 0.109 & 1.000 & -0.249 & -0.027 & 0.002 & $1.01 \mathrm{E}-04$ & $-8.84 \mathrm{E}-06$ & 2.33E-08 \\
\hline & & Mean & -1.095 & 0.108 & 0.152 & -0.085 & -0.028 & -0.254 & $-1.34 \mathrm{E}-02$ & $-1.72 \mathrm{E}-01$ & $-1.11 \mathrm{E}-05$ \\
\hline & & Worst & -0.965 & 0.216 & -0.663 & 0.663 & -0.028 & -3.408 & $-9.60 \mathrm{E}-02$ & $-1.12 \mathrm{E}+00$ & $-8.63 \mathrm{E}-05$ \\
\hline & $\widehat{0.010^{2}}$ & Best & -1.094 & 0.109 & 1.000 & -0.249 & -0.027 & 0.002 & $9.67 \mathrm{E}-05$ & $-9.73 \mathrm{E}-06$ & 2.32E-08 \\
\hline & & Mean & -1.090 & 0.112 & 0.056 & -0.019 & -0.028 & -0.365 & $-1.39 \mathrm{E}-02$ & $-2.10 \mathrm{E}-01$ & $-1.19 \mathrm{E}-05$ \\
\hline & & Worst & -0.955 & 0.224 & -0.559 & 0.559 & -0.028 & -3.498 & $-1.28 \mathrm{E}-01$ & $-1.49 \mathrm{E}+00$ & $-1.01 \mathrm{E}-04$ \\
\hline & $0.100^{2}$ & Best & -1.092 & 0.109 & -0.173 & 0.173 & -0.028 & -0.337 & $-2.13 \mathrm{E}-02$ & $-4.24 \mathrm{E}-01$ & $-2.87 \mathrm{E}-05$ \\
\hline & & Mean & -1.087 & 0.114 & 0.066 & -0.021 & -0.029 & -0.285 & $-8.76 \mathrm{E}-03$ & $-1.83 \mathrm{E}-01$ & $-1.26 \mathrm{E}-05$ \\
\hline & & Worst & -1.017 & 0.170 & -0.409 & 0.409 & -0.028 & -1.699 & $-8.96 \mathrm{E}-02$ & $-1.10 \mathrm{E}+00$ & $-6.33 \mathrm{E}-05$ \\
\hline \multicolumn{3}{|c|}{$\overline{\mathrm{eV}}$} & -1.094 & 0.109 & 1.000 & -0.249 & 28 & 0.002 & $-7.83 \mathrm{E}-06$ & $1.78 \mathrm{E}-08$ & $2.36 \mathrm{E}-08$ \\
\hline
\end{tabular}

The performance of the DE approach for ESM models is also investigated through single objective based merit function given in (18) and results in terms of the learning curves are given in Fig. 13. While, the detailed evaluation of the accuracy of the algorithm using single objective based merit function given in (18) is carried out by statistical performance measures of mean, best, and worst magnitudes of the estimated parameters and results are presented in Tables 12, 13 and 14 for ESMI, ESM-II and ESM-III respectively. It is seen from the convergence plots presented in Fig. 13 that minimum error in the responses of the ESM models I, II and III can be achieved by using objective 
function (18) but convergence to the desired parameters is not guaranteed as can be seen from Tables 12 to 14 for ESM-I, ESM-II and ESM-III respectively.

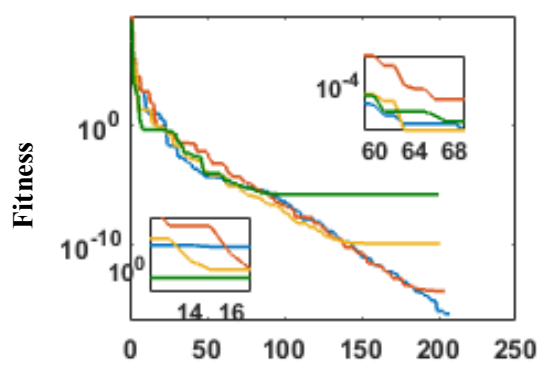

(a) DE learning curves for ESM-I with single objective (18)

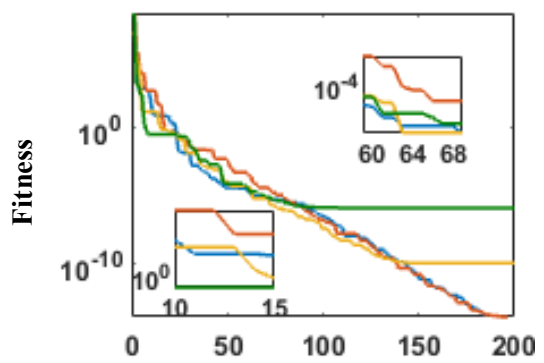

(b) DE learning curves for ESM-II with single objective (18)

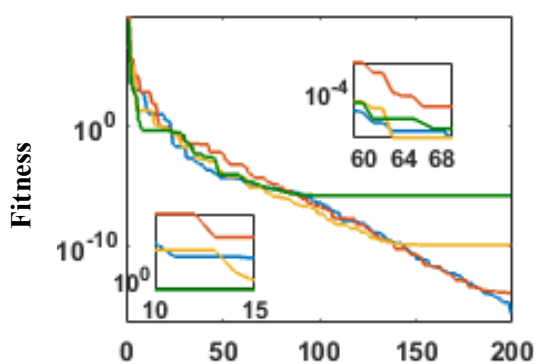

(c) DE learning curves for ESM-III with single objective (18)

$$
\longrightarrow \sigma^{2}=0^{2}-\sigma^{2}=0.001^{2}-\sigma^{2}=0.01^{2}-\sigma^{2}=0.1^{2}
$$

Fig. 13. Results for iterative adaptation using single objective based merit function of ESM-I, II and III

Table 12: Comparison through statistical results for variants of ESM-I system using single objective based merit function

\begin{tabular}{|c|c|c|c|c|c|c|c|c|}
\hline \multirow{2}{*}{ Solver } & \multirow{2}{*}{$\sigma^{2}$} & \multirow{2}{*}{ Model } & \multicolumn{6}{|c|}{ Approximate Parameter Vector } \\
\hline & & & $i=1$ & $i=2$ & $i=\mathbf{3}$ & $i=4$ & $i=5$ & $i=6$ \\
\hline \multirow{12}{*}{ DE } & \multirow{3}{*}{$0^{2}$} & Best & -1.493 & 0.383 & -0.001 & 0.237 & -0.012 & 6.774 \\
\hline & & Mean & -0.869 & 0.586 & -0.015 & 0.028 & 0.000 & 3.395 \\
\hline & & Worst & 1.529 & 1.349 & -0.008 & 0.028 & 0.000 & 1.837 \\
\hline & \multirow{3}{*}{$10^{-4}$} & Best & -1.743 & 0.679 & 0.017 & -0.006 & -0.047 & 9.804 \\
\hline & & Mean & -0.855 & 0.615 & -0.002 & -0.004 & -0.080 & 2.253 \\
\hline & & Worst & 1.521 & 1.099 & -0.035 & 0.000 & -0.859 & -0.950 \\
\hline & \multirow{3}{*}{$10^{-2}$} & Best & -2.288 & 1.342 & 0.155 & 0.399 & 0.024 & 79.459 \\
\hline & & Mean & -1.314 & 0.698 & -0.046 & 0.049 & -0.158 & 9.897 \\
\hline & & Worst & -0.237 & -1.056 & -0.018 & 0.001 & -0.197 & -0.970 \\
\hline & \multirow{3}{*}{$10^{-1}$} & Best & -1.958 & 0.940 & -0.013 & 0.224 & -0.911 & 1.423 \\
\hline & & Mean & -1.750 & 1.161 & 0.001 & 0.138 & 3.063 & 1.228 \\
\hline & & Worst & -0.130 & -1.214 & -0.044 & 0.038 & 10.191 & -3.005 \\
\hline \multicolumn{2}{|c|}{ True Parameter } & tor & -1.998 & 0.998 & 0.002 & 6.899 & 0.041 & 2389.700 \\
\hline
\end{tabular}


Table 13: Comparison through statistical results for variants of ESM-II system using single objective based merit function

\begin{tabular}{|c|c|c|c|c|c|c|c|c|c|}
\hline \multirow{2}{*}{ Solver } & \multirow{2}{*}{ Noise } & \multirow{2}{*}{ Model } & \multicolumn{7}{|c|}{ Approxi mate Parameter Vector } \\
\hline & & & $i=1$ & $i=2$ & $i=\mathbf{3}$ & $i=4$ & $i=5$ & $i=6$ & $i=7$ \\
\hline \multirow{12}{*}{ DE } & $0.000^{2}$ & Best & 0.000 & 0.383 & -0.001 & 0.237 & -0.012 & 6.774 & 0.774 \\
\hline & & Mean & -0.869 & 0.586 & -0.015 & 0.028 & 0.000 & 3.395 & 1.321 \\
\hline & & Worst & 1.529 & 1.349 & -0.008 & 0.028 & 0.000 & 1.837 & -1.363 \\
\hline & $0.001^{2}$ & Best & -1.743 & 0.679 & 0.017 & -0.006 & -0.047 & 9.804 & 0.880 \\
\hline & & Mean & -0.855 & 0.615 & -0.002 & -0.004 & -0.080 & 2.253 & 1.450 \\
\hline & & Worst & 1.521 & 1.099 & -0.035 & 0.000 & -0.859 & -0.950 & 2.372 \\
\hline & $0.010^{2}$ & Best & -2.288 & 1.342 & 0.155 & 0.399 & 0.024 & 79.459 & 1.068 \\
\hline & & Mean & -1.314 & 0.698 & -0.046 & 0.049 & -0.158 & 9.897 & 1.868 \\
\hline & & Worst & -0.237 & -1.056 & -0.018 & 0.001 & -0.197 & -0.970 & 2.787 \\
\hline & $0.100^{2}$ & Best & -1.958 & 0.940 & -0.013 & 0.224 & -0.911 & 1.423 & 1.106 \\
\hline & & Mean & -1.750 & 1.161 & 0.001 & 0.138 & 3.063 & 1.228 & 1.442 \\
\hline & & Worst & -0.130 & -1.214 & -0.044 & 0.038 & 10.191 & -3.005 & 2.122 \\
\hline \multicolumn{3}{|c|}{ True Values } & -1.000 & 0.800 & 1.000 & 2.800 & -4.800 & 5.700 & 0.600 \\
\hline
\end{tabular}

Table 14: Comparison through statistical results for variants of ESM-III system using single objective based merit function

\begin{tabular}{|c|c|c|c|c|c|c|c|c|c|c|c|}
\hline \multirow{2}{*}{ Solver } & \multirow{2}{*}{ Noise } & \multirow{2}{*}{ Model } & \multicolumn{9}{|c|}{ Approxi mate Parameter Vector } \\
\hline & & & $i=1$ & $i=2$ & $i=\mathbf{3}$ & $i=4$ & $i=5$ & $i=6$ & $i=7$ & $i=8$ & $i=9$ \\
\hline \multirow{12}{*}{ DF } & 002 & Best & -1.094 & 0.109 & -0.004 & 0.001 & 4.621 & -0.454 & $1.73 \mathrm{E}-03$ & $-5.09 \mathrm{E}-06$ & $-5.51 \mathrm{E}-06$ \\
\hline & & Mean & -1.094 & 0.110 & -0.170 & 0.170 & -5730.349 & 114.328 & $-7.63 \mathrm{E}-01$ & $1.69 \mathrm{E}-03$ & $1.69 \mathrm{E}-03$ \\
\hline & & Worst & -1.098 & 0.113 & -1.695 & 1.697 & -57575.591 & 1151.848 & $-7.68 \mathrm{E}+00$ & $1.70 \mathrm{E}-02$ & 1.71E-02 \\
\hline & $\overline{01^{2}}$ & Best & -1.094 & 0.109 & 0.002 & -0.001 & 3.090 & 0.501 & $-1.28 \mathrm{E}-03$ & $2.05 \mathrm{E}-05$ & $5.52 \mathrm{E}-06$ \\
\hline & & Mean & -1.094 & 0.110 & -0.221 & 0.221 & -85680.575 & 713.603 & $-1.14 \mathrm{E}+01$ & $2.54 \mathrm{E}-02$ & $2.54 \mathrm{E}-02$ \\
\hline & & Worst & -1.098 & 0.113 & -2.204 & 2.207 & -857185.653 & 17144.022 & $-1.14 \mathrm{E}+02$ & $2.54 \mathrm{E}-01$ & $2.54 \mathrm{E}-01$ \\
\hline & $\overline{0.010^{2}}$ & Best & -1.094 & 0.109 & 0.001 & 0.000 & 2.768 & 1.279 & $3.86 \mathrm{E}-03$ & $3.52 \mathrm{E}-03$ & $1.47 \mathrm{E}-05$ \\
\hline & & Mean & -1.094 & 0.110 & -0.131 & 0.131 & 0.016 & -323.224 & $2.16 \mathrm{E}+00$ & $-3.34 \mathrm{E}-03$ & $-4.79 \mathrm{E}-03$ \\
\hline & & Worst & -1.098 & 0.113 & -1.308 & 1.309 & 0.016 & -3236.617 & $2.16 \mathrm{E}+01$ & $-4.80 \mathrm{E}-02$ & $-4.79 \mathrm{E}-02$ \\
\hline & $0.100^{2}$ & Best & -1.086 & 0.106 & 0.002 & 0.002 & -0.094 & 18.973 & $-8.10 \mathrm{E}-02$ & $-2.64 \mathrm{E}-01$ & 2.82E-04 \\
\hline & & 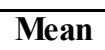 & -1.086 & 0.106 & 0.000 & 0.000 & -0.014 & 3.070 & 3.31E-02 & $-3.11 \mathrm{E}-01$ & 4.64E-05 \\
\hline & & t & -1.086 & 0.106 & 0.001 & 0.001 & -0.098 & 2.283 & 7.20E-02 & $-5.06 \mathrm{E}-01$ & $3.53 \mathrm{E}-05$ \\
\hline \multicolumn{3}{|c|}{ True Values } & -1.094 & 0.109 & 1.000 & -0.249 & -0.028 & 0.002 & $-7.83 \mathrm{E}-06$ & $1.78 \mathrm{E}-08$ & 2.36E-08 \\
\hline
\end{tabular}

\section{Conclusions}

Evolutionary computing paradigm based on DE is exploited as an accurate, reliable, robust and efficient tool for parameter estimation of Hammerstein systems in ESM model representing the scenarios of rehabilitation of the stroke patients. Hammerstein control autoregressive structure of ESM systems based on nonlinear characteristics of polynomial, sigmoid and spline kernels. Optimization strength of DE is employed for three ESM case studies for noiseless and noisy scenarios having variance $\sigma^{2}=0.001^{2}, 0.01^{2}$, and $0.1^{2}$. Comparative study of the proposed method from GAs, PSO, PS and SA based on performance metrics of $M A E_{\vartheta}, E N S E_{\vartheta}$ and $T_{\vartheta} C_{\vartheta}$ demonstrated the efficacy of DE in each scenarios of ESM models, while, the accuracy of estimation of both algorithm degraded for higher noise variances, but still the DE outperformed its 
counterpart GA, PSO, PS and SA in terms of accuracy and complexity measures. Statistical observations further verify and validate the worth of DE over its counterparts for each scenario of ESM model.

One may explore in meta-heuristics of backtracking search optimization, weighted differential evolutions, fireworks, firefly, grey wolf optimizer, covariance matrix adaptation evolution strategy and improved environmental adaptation method for better identification of ESM models.

\section{References}

[1] Castro, M. J., Apple Jr, D. F., Hillegass, E. A., \& Dudley, G. A. (1999). Influence of complete spinal cord injury on skeletal muscle cross-sectional area within the first 6 months of injury. European Journal of Applied Physiology and Occupational Physiology, 80(4), 373-378.

[2] Vestergaard, P., Krogh, K., Rejnmark, L., \& Mosekilde, L. (1998). Fracture rates and risk factors for fractures in patients with spinal cord injury. Spinal cord, 36(11), 790.

[3] Mahoney, E. T., Bickel, C. S., Elder, C., Black, C., Slade, J. M., Apple, D., \& Dudley, G. A. (2005). Changes in skeletal muscle size and glucose tolerance with electrically stimulated resistance training in subjects with chronic spinal cord injury. Archives of physical medicine and rehabilitation, 86(7), 1502-1504.

[4] Shields, R. K., \& Dudley-Javoroski, S. (2007). Musculoskeletal adaptations in chronic spinal cord injury: effects of long-term soleus electrical stimulation training. Neurorehabilitation and neural repair, 21(2), 169-179.

[5] Howlett, O. A., Lannin, N. A., Ada, L., \& McKinstry, C. (2015). Functional electrical stimulation improves activity after stroke: a systematic review with metaanalysis. Archives of physical medicine and rehabilitation, 96(5), 934-943.

[6] Haeufle, D. F. B., Günther, M., Bayer, A., \& Schmitt, S. (2014). Hill-type muscle model with serial damping and eccentric force-velocity relation. Journal of biomechanics, 47(6), 1531-1536.

[7] Law, L. F., \& Shields, R. K. (2007). Mathematical models of human paralyzed muscle after long-term training. Journal of biomechanics, 40(12), 2587-2595.

[8] Ding, J., Wexler, A. S., \& Binder-Macleod, S. A. (2003). Mathematical models for fatigue minimization during functional electrical stimulation. Journal of Electromyography and Kinesiology, 13(6), 575-588.

[9] Hunt, K. J., Munih, M., Donaldson, N. D. N., \& Barr, F. M. (1998). Investigation of the Hammerstein hypothesis in the modeling of electrically stimulated muscle. IEEE Transactions on Biomedical Engineering, 45(8), 998-1009.

[10] Bai, E. W., Cai, Z., Dudley-Javorosk, S., \& Shields, R. K. (2009). Identification of a modified Wiener-Hammerstein system and its application in electrically stimulated paralyzed skeletal muscle modeling. Automatica, 45(3), 736-743.

[11] Le, F. (2011). Identification of electrically stimulated muscle after stroke Doctoral dissertation, University of Southampton. 
[12] Le, F., Markovsky, I., Freeman, C. T., \& Rogers, E. (2012). Recursive identification of Hammerstein systems with application to electrically stimulated muscle. Control Engineering Practice, 20(4), 386-396.

[13] Mehmood, A., Zameer, A., Chaudhary, N. I., \& Raja, M. A. Z. (2019). Backtracking search heuristics for identification of electrical muscle stimulation models using Hammerste in structure. Applied Soft Computing, 84, 105705.

[14] Greblicki, W., \& Pawlak, M. (2017). Hammerstein system identification with the nearest neighbor algorithm. IEEE Transactions on Information Theory, 63(8), 4746-4757.

[15] Castro-Garcia, R., Agudelo, O. M., \& Suykens, J. A. (2017). Impulse response constrained LS-SVM modelling for MIMO Hammerstein system identification. International Journal of Control, 1-18.

[16] Giordano, G., Gros, S., \& Sjöberg, J. (2018). An improved method for WienerHammerstein system identification based on the Fractional Approach. Automatica, 94, 349-360.

[17] Wang, D. (2016). Hierarchical parameter estimation for a class of MIMO Hammerste in systems based on the reframed models. Applied Mathematics Letters, 57, 13-19..

[18] Chen, H., \& Ding, F. (2015). Hierarchical least squares identification for Hammerste in nonlinear controlled autoregressive systems. Circuits, Systems, and Signal Processing, 34(1), 61-75.

[19] Mao, Y., Ding, F., \& Yang, E. (2017). Adaptive filtering-based multi-innovation gradient algorithm for input nonlinear systems with autoregressive noise. International Journal of Adaptive Control and Signal Processing, 31(10), 1388-1400.

[20] Mao, Y., \& Ding, F. (2015). Multi-innovation stochastic gradient identification for Hammerstein controlled autoregressive autoregressive systems based on the filtering technique. Nonlinear Dynamics, 79(3), 1745-1755.

[21] Ding, F., Wang, F., Xu, L., Hayat, T., \& Alsaedi, A. (2016). Parameter estimation for pseudo-linear systems using the auxiliary model and the decomposition technique. IET Control Theory \& Applications, 11(3), 390-400.

[22] Mao, Y., \& Ding, F. (2016). A novel parameter separation based identification algorithm for Hammerstein systems. Applied Mathematics Letters, 60, 21-27.

[23] Chaudhary, N. I., et al, (2015). Design of fractional adaptive strategy for input nonlinear Box-Jenkins systems. Signal Processing, 116, 141-151.

[24] Chaudhary, N. I., et al., (2015). Design of modified fractional adaptive strategies for Hammerstein nonlinear control autoregressive systems. Nonlinear Dynamics, 82(4), 18111830.

[25] Cheng, S., Wei, Y., Sheng, D., Chen, Y., \& Wang, Y. (2018). Identification for Hammerstein nonlinear ARMAX systems based on multi-innovation fractional order stochastic gradient. Signal Processing, 142, 1-10.

[26] Chaudhary, N. I., et al., (2015). Identification of Hammerstein nonlinear ARMAX systems using nonlinear adaptive algorithms. Nonlinear Dynamics, 79(2), 1385-1397. 
[27] Aslam, M. S., et al., (2017). A sliding-window approximation-based fractional adaptive strategy for Hammerstein nonlinear ARMAX systems. Nonlinear Dynamics, 87(1), 519533.

[28] Chaudhary, N. I., et al., (2017). Modified Volterra LMS algorithm to fractional order for identification of Hammerstein non-linear system. IET Signal Processing, 11(8), 975-985.

[29] Dienouri, Y., Belhadi, A., \& Belkebir, R. (2018). Bees swarm optimization guided by data mining techniques for document information retrieval. Expert Systems With Applications, 94, 126-136.

[30] Dhiman, G., \& Kumar, V. (2018). Emperor Penguin Optimizer: A Bio-inspired Algorithm for Engineering Problems. Knowledge-Based Systems.

[31] Bose, D., Biswas, S., Vasilakos, A. V., \& Laha, S. (2014). Optimal filter design using an improved artificial bee colony algorithm. Information Sciences, 281, 443-461.

[32] Abiyev, R. H., \& Tunay, M. (2015). Optimization of high-dimensional functions through hypercube evaluation. Computational intelligence and neuroscience, 2015, 17.

[33] Zhao, W., Wang, L., \& Zhang, Z. (2018). Atom search optimization and its application to solve a hydrogeologic parameter estimation problem. Knowledge-Based Systems.

[34] Chen, X., Mei, C., Xu, B., Yu, K., \& Huang, X. (2018). Quadratic interpolation based teaching-learning-based optimization for chemical dynamic system optimization. Knowledge-Based Systems, 145, 250-263.

[35] Lodhi, S., et al., 2019. Fractional neural network models for nonlinear Riccati systems. Neural Computing and Applications, 31(1), pp.359-378.

[36] Kumar, A. and Kumar, V., 2018. Performance analysis of optimal hybrid novel interval type-2 fractional order fuzzy logic controllers for fractional order systems. Expert Systems with Applications, 93, pp.435-455.

[37] Raja, M. A. Z., Abbas, S., Syam, M. I., \& Wazwaz, A. M. (2018). Design of neuroevolutionary model for solving nonlinear singularly perturbed boundary value problems. Applied Soft Computing, 62, 373-394.

[38] Raja, M. A. Z. (2014). Numerical treatment for boundary value problems of pantograph functional differential equation using computational intelligence algorithms. Applied Soft Computing, 24, 806-821.

[39] Umar, M., et al, 2019. Intelligent computing for numerical treatment of nonlinear preypredator models. Applied Soft Computing, 80, pp.506-524.

[40] Wang, Y., Feng, X., Lyu, X., Li, Z. and Liu, B., 2016. Optimal targeting of nonlinear chaotic systems using a novel evolutionary computing strategy. Knowledge-Based Systems, 107, pp.261-270.

[41] Chen, K., Zhou, F. and Liu, A., 2018. Chaotic dynamic weight particle swarm optimization for numerical function optimization. Knowledge-Based Systems, 139, pp.23-40.

[42] Ahmad, I., et al., (2018). Neuro-evolutionary computing paradigm for Painlevé equationII in nonlinear optics. The European Physical Journal Plus, 133(5), 184. 
[43] Raja, M. A. Z., Shah, Z., Manzar, M. A., Ahmad, I., Awais, M., \& Baleanu, D. (2018). A new stochastic computing paradigm for nonlinear Painlevé II systems in applications of random matrix theory. The European Physical Journal Plus, 133(7), 254.

[44] Raja, M. A. Z., Shah, F. H., Khan, A. A., \& Khan, N. A. (2016). Design of bio-inspired computational intelligence technique for solving steady thin film flow of JohnsonSegalman fluid on vertical cylinder for drainage problems. Journal of the Taiwan Institute of Chemical Engineers, 60, 59-75.

[45] Ahmad, I., et al., 2019. Design of computational intelligent procedure for thermal analysis of porous fin model. Chinese Journal of Physics, 59, pp.641-655.

[46] Mehmood, A., et al., (2018). Parameter estimation for Hammerstein control autoregressive systems using differential evolution. Signal, Image and Video Processing, 12(8), 16031610.

[47] Raja, M. A. Z., Shah, A. A., Mehmood, A., Chaudhary, N. I., \& Aslam, M. S. (2018). Bioinspired computational heuristics for parameter estimation of nonlinear Hammerste in controlled autoregressive system. Neural Computing and Applications, 29(12), 14551474.

[48] Khan, W. U., et al., (2018). Backtracking search integrated with sequential quadratic programing for nonlinear active noise control systems. Applied Soft Computing, 73, 666683.

[49] Khan, W.U., et al, 2019. A novel application of fireworks heuristic paradigms for reliable treatment of nonlinear active noise control. Applied Acoustics, 146, pp.246-260.

[50] Mehmood, A., et al., (2019). Nature-inspired heuristic paradigms for parameter estimation of control autoregressive moving average systems. Neural Computing and Applications, 31(10), 5819-5842.

[51] Ahmad, I., et al., (2016). Bio-inspired computational heuristics to study Lane-Emden systems arising in astrophysics model. SpringerPlus, 5(1), 1866.

[52] Pathak, M., \& Joshi, P. (2018). Application of a coupled approach for the solution of nonlinear singular initial value problems of Lane-Emden type. Astrophysics and Space Science, 363(9), 191.

[53] Raja, M. A. Z., Zameer, A., Khan, A. U., \& Wazwaz, A. M. (2016). A new numerical approach to solve Thomas-Fermi model of an atom using bio-inspired heuristics integrated with sequential quadratic programming. SpringerPlus, 5(1), 1400.

[54] Sabir, Z., et al., (2018). Neuro-heuristics for nonlinear singular Thomas-Fermi systems. Applied Soft Computing, 65, 152-169.

[55] Raja, M. A. Z., Shah, F. H., Tariq, M., \& Ahmad, I. (2018). Design of artificial neural network models optimized with sequential quadratic programming to study the dynamics of nonlinear Troesch's problem arising in plasma physics. Neural Computing and Applications, 29(6), 83-109.

[56] Majeed, K., et al., (2017). A genetic algorithm optimized Morlet wavelet artificial neural network to study the dynamics of nonlinear Troesch's system. Applied Soft Computing, 56, 420-435. 
[57] Ahmad, I., et al., (2017). Neural network methods to solve the Lane-Emden type equations arising in thermodynamic studies of the spherical gas cloud model. Neural Computing and Applications, 28(1), 929-944.

[58] Raja, M. A. Z., Niazi, S. A., \& Butt, S. A. (2017). An intelligent computing technique to analyze the vibrational dynamics of rotating electrical machine. Neurocomputing, 219, 280-299.

[59] Vitayasak, S., \& Pongcharoen, P. (2018). Performance improvement of TeachingLearning-Based Optimisation for robust machine layout design. Expert Systems with Applications, 98, 129-152.

[60] Mehmood, A., et al., 2018. Design of neuro-computing paradigms for nonlinear nanofluidic systems of MHD Jeffery-Hamel flow. Journal of the Taiwan Institute of Chemical Engineers, 91, pp.57-85.

[61] Raja, M. A. Z., Ahmed, T., \& Shah, S. M. (2017). Intelligent computing strategy to analyze the dynamics of convective heat transfer in MHD slip flow over stretching surface involving carbon nanotubes. Journal of the Taiwan Institute of Chemical Engineers, 80, 935-953.

[62] Mehmood, A., et al., Design of nature-inspired heuristic paradigm for systems in nonlinear electrical circuits. Neural Computing and Applications, pp.1-17.

[63] Raja, M. A. Z., Mehmood, A., Niazi, S. A., \& Shah, S. M. 2018. Computational intellige nce methodology for the analysis of RC circuit modelled with nonlinear differential order system. Neural Computing and Applications, 30(6), pp. 1905-1924.

[64] Li, Y. Z., Jiang, L., Wu, Q. H., Wang, P., Gooi, H. B., Li, K. C., ... \& Imura, J. (2017). Wind-thermal power system dispatch using MLSAD model and GSOICLW algorithm. Knowledge-Based Systems, 116, 94-101.

[65] Nuaekaew, K., Artrit, P., Pholdee, N., \& Bureerat, S. (2017). Optimal reactive power dispatch problem using a two-archive multi-objective grey wolf optimizer. Expert Systems with Applications, 87, 79-89.

[66] Mehmood, A., et al, Novel computing paradigms for parameter estimation in power signal models. Neural Computing and Applications, pp.1-30.

[67] Zameer, A., et al, (2017). Intelligent and robust prediction of short term wind power using genetic programming based ensemble of neural networks. Energy conversion and management, 134, 361-372.

[68] Kelly, S., \& Ahmad, K. (2018). Estimating the impact of domain-specific news sentiment on financial assets. Knowledge-Based Systems, 150, 116-126.

[69] Ara, A., et al., 2018. Wavelets optimization method for evaluation of fractional partial differential equations: an application to financial modelling. Advances in Difference Equations, 2018(1), p.8.

[70] Karhunen, M. (2019). Algorithmic sign prediction and covariate selection across eleven international stock markets. Expert Systems with Applications, 115, 256-263. 
[71] Cerqueti, R., Ferraro, G., \& Iovanella, A. (2018). A new measure for community structures through indirect social connections. Expert Systems with Applications, 114, 196-209.

[72] Raja, M. A. Z., Asma, K., \& Aslam, M. S. (2018). Bio-inspired computational heuristics to study models of hiv infection of $\mathrm{CD} 4+\mathrm{T}$-cell. International Journal of Biomathematics, 11(02), 1850019.

[73] Raja, M. A. Z., Shah, F. H., Alaidarous, E. S., \& Syam, M. I. (2017). Design of bio-inspired heuristic technique integrated with interior-point algorithm to analyze the dynamics of heartbeat model. Applied Soft Computing, 52, 605-629.

[74] Storn, R., \& Price, K. (1997). Differential evolution-a simple and efficient heuristic for global optimization over continuous spaces. Journal of global optimization, 11(4), 341359.

[75] Price, K., Storn, R. M., \& Lampinen, J. A. (2006). Differential evolution: a practical approach to global optimization. Springer Science \& Business Media.

[76] Buba, A. T., \& Lee, L. S. (2018). A differential evolution for simultaneous transit network design and frequency setting problem. Expert Systems with Applications, 106, 277-289.

[77] Mlakar, U., Fister, I., Brest, J., \& Potočnik, B. (2017). Multi-objective differential evolution for feature selection in facial expression recognition systems. Expert Systems with Applications, 89, 129-137.

[78] Li, H., Gong, M., Wang, C., \& Miao, Q. (2018). Self-paced stacked denoising autoencoders based on differential evolution for change detection. Applied Soft Computing, 71, 698-714.

[79] Sayah, S. (2018). Modified differential evolution approach for practical optimal reactive power dispatch of hybrid AC- DC power systems. Applied Soft Computing.

[80] Vali, M. H., Aghagolzadeh, A., \& Baleghi, Y. (2018). Optimized watermarking technique using self-adaptive differential evolution based on redundant discrete wavelet transform and singular value decomposition. Expert Systems with Applications, 114, 296-312.

[81] Hancer, E., Xue, B., \& Zhang, M. (2018). Differential evolution for filter feature selection based on information theory and feature ranking. Knowledge-Based Systems, 140, 103119.

[82] Holland, J. H. (1992). Genetic algorithms. Scientific american, 267(1), 66-73.

[83] Davis, L. (1991). Handbook of genetic algorithms.

[84] Keshavarz, H., \& Abadeh, M. S. (2017). ALGA: Adaptive lexicon learning using genetic algorithm for sentiment analysis of microblogs. Knowledge-Based Systems, 122, 1-16.

[85] Raman, M. G., Somu, N., Kirthivasan, K., Liscano, R., \& Sriram, V. S. (2017). An effic ient intrusion detection system based on hypergraph-Genetic algorithm for parameter optimization and feature selection in support vector machine. Knowledge-Based Systems, 134, 1-12.

[86] Tseng, H. E., Chang, C. C., Lee, S. C., \& Huang, Y. M. (2018). A Block-based genetic algorithm for disassembly sequence planning. Expert Systems with Applications, 96, 492505. 
[87] Owais, M., \& Osman, M. K. (2018). Complete hierarchical multi-objective genetic algorithm for transit network design problem. Expert Systems with Applications, 114, 143154.

[88] Jadhav, S., He, H., \& Jenkins, K. (2018). Information gain directed genetic algorithm wrapper feature selection for credit rating. Applied Soft Computing, 69, 541-553.

[89] Raja, M. A. Z., Mehmood, A., ur Rehman, A., Khan, A., \& Zameer, A. (2018). Bio-insp ired computational heuristics for Sisko fluid flow and heat transfer models. Applied Soft Computing, 71, 622-648.

[90] Chouhdry, Z.R., et al., 2018. Design of reduced search space strategy based on integration of Nelder-Mead method and pattern search algorithm with application to economic load dispatch problem. Neural Computing and Applications, 30(12), pp.3693-3705.

[91] Raja, M.A.Z. and Samar, R., 2014. Solution of the 2-dimensional Bratu problem using neural network, swarm intelligence and sequential quadratic programming. Neural Computing and applications, 25(7-8), pp.1723-1739.

[92] Raja, M.A.Z., Khan, J.A. and Qureshi, I.M., 2010. A new stochastic approach for solution of Riccati differential equation of fractional order. Annals of Mathematics and Artificial Intelligence, 60(3-4), pp.229-250. 